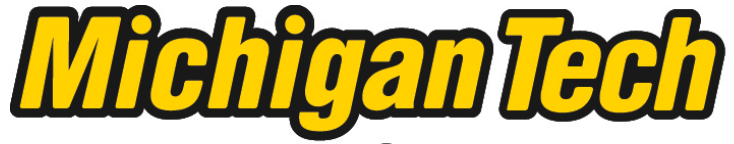 \\ Michigan Technological University Create the Future Digital Commons @ Michigan Tech
}

Dissertations, Master's Theses and Master's Reports - Open

Dissertations, Master's Theses and Master's

Reports

2015

\section{AN ANALYSIS OF MULTIPLICATIVE REGULARIZATION}

Elaheh Gorgin

Michigan Technological University

Follow this and additional works at: https://digitalcommons.mtu.edu/etds

Part of the Applied Mathematics Commons

Copyright 2015 Elaheh Gorgin

\section{Recommended Citation}

Gorgin, Elaheh, "AN ANALYSIS OF MULTIPLICATIVE REGULARIZATION", Dissertation, Michigan Technological University, 2015.

https://doi.org/10.37099/mtu.dc.etds/1017

Follow this and additional works at: https://digitalcommons.mtu.edu/etds

Part of the Applied Mathematics Commons 
AN ANALYSIS OF MULTIPLICATIVE REGULARIZATION

By

Elaheh Gorgin

\begin{abstract}
A DISSERTATION
Submitted in partial fulfillment of the requirements for the degree of DOCTOR OF PHILOSOPHY
\end{abstract}

In Mathematical Sciences

MICHIGAN TECHNOLOGICAL UNIVERSITY

2015

(C) 2015 Elaheh Gorgin 

This dissertation has been approved in partial fulfillment of the requirements for the Degree of DOCTOR OF PHILOSOPHY in Mathematical Sciences.

Department of Mathematical Sciences

Dissertation Advisor: Dr. Mark S. Gockenbach

Committee Member: Dr. Jiguang Sun

Committee Member: Dr. Tamara Olson

Committee Member: Dr. Seyyedmohsen Azizi

Department Chair: Dr. Mark S. Gockenbach 



\section{Dedicated}

To my mother, Sedigheh 



\section{Contents}

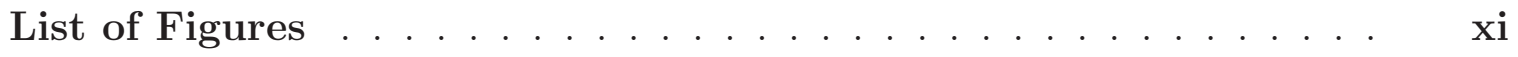

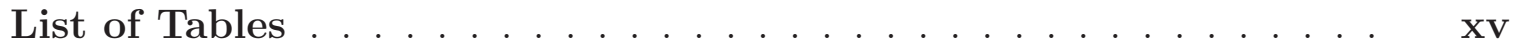

Acknowledgments .................. xvii

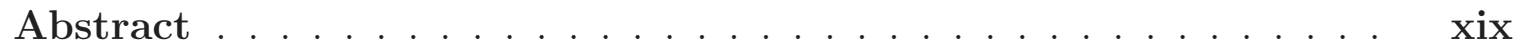

1 Introduction . . . . . . . . . . . . . . . . . . 1

1.1 Linear inverse problems . . . . . . . . . . . . . . . . . . 1

1.1.1 Well-posed and ill-posed problems . . . . . . . . . 2

1.1.2 Dealing with lack of existence and lack of uniqueness . . . 3

1.1.3 The Moore-Penrose generalized inverse . . . . . . . . 5

1.1.4 Compact operators . . . . . . . . . . . . 7

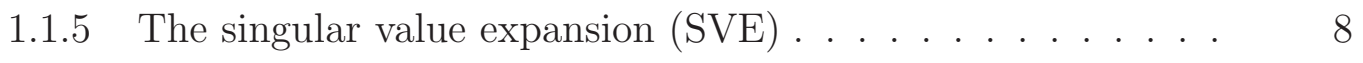

1.1.6 Fredholm integral equations . . . . . . . . . . . . . 10

1.2 Tikhonov regularization and parameter choice rules $\ldots \ldots \ldots$

1.2.1 Definitions and basic properties . . . . . . . . . . 13

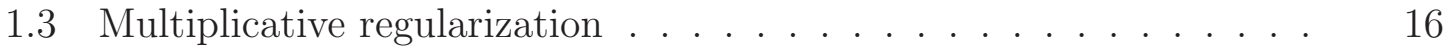


1.4 The L-curve criterion . . . . . . . . . . . . . . . . . . . . . . 18

1.4.1 Definitions of the L-curve . . . . . . . . . . . 18

1.5 Regularization and finite-dimensional problems . . . . . . . . 20

1.5.1 The singular value decomposition (SVD) . . . . . . . 20

1.5.2 The comparison between SVE and SVD . . . . . . . . 21

1.5.3 Tikhonov regularization method . . . . . . . . . . . . . 21

1.6 Test problems . . . . . . . . . . . . . . . . . . . . . . . . 23

1.6.1 The degree of ill-posedness . . . . . . . . . . . . 26

1.7 Outline of dissertation . . . . . . . . . . . . . . . . . . . . . 28

2 Literature review . . . . . . . . . . . . . . . . . . . . . 31

2.1 Multiplicative regularization . . . . . . . . . . . . . . 31

2.2 Popular parameter choice rules . . . . . . . . . . . . . . . . . 32

2.2.1 Morozov discrepancy principle . . . . . . . . . . . . . 32

2.2.2 Generalized cross-validation method . . . . . . . . . . . 33

2.3 The L-curve method . . . . . . . . . . . . . . . . . . . . . . 34

2.3.1 The definition of the corner . . . . . . . . . . . 34

2.3.2 Concavity of the L-curve . . . . . . . . . . . . . . 35

2.3.3 Other disadvantages of the L-curve . . . . . . . . . . 37

2.3.4 Rate of convergence . . . . . . . . . . . . . . . 39

2.4 Conclusions . . . . . . . . . . . . . . . . . . . . . . . . . . . . . . . . . . . . 39

3 Multiplicative regularization for finite-dimensional problems . . 41 
3.1 Notation and assumption . . . . . . . . . . . . . . . . 42

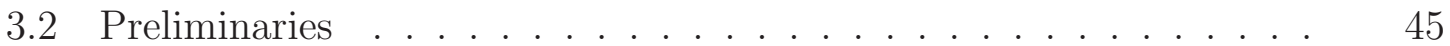

3.3 Analysis of multiplicative regularization . . . . . . . . . . 53

3.4 Rate of convergence . . . . . . . . . . . . . . . . 62

3.4.1 Numerical examples . . . . . . . . . . . . . . . . . 64

3.5 Comparison with the L-curve method . . . . . . . . . . . . . . 65

3.5.1 The failure of multiplicative regularization on "deriv2" and "phillips" test problems . . . . . . . . . . . . . 70

3.5.2 More details about the numerical examples . . . . . . . . . . 71

3.6 Conclusions ........................ 76

4 Multiplicative regularization for infinite-dimensional problems . 79

4.1 Analysis of multiplicative regularization for infinite-dimensional prob-

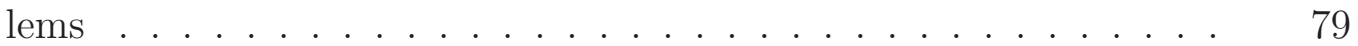

4.2 Rate of convergence . . . . . . . . . . . . 86

4.2.1 Special cases . . . . . . . . . . . . . . . . 88

4.2.2 Counter-example .................. . . 90

4.2.3 Numerical experiments . . . . . . . . . . . . . . . . . . . 92

4.3 Conclusions . . . . . . . . . . . . . . . . . . . . . 97

5 Conclusions . . . . . . . . . . . . . . . . . . 99

5.1 Summary and conclusions . . . . . . . . . . . . . . . 99

5.2 Future work: plans and goals . . . . . . . . . . . . . . 101 


\section{List of Figures}

1.1 The L-curve plot of the test problem "deriv2" $(\mathrm{n}=100)$ where the noise level is $\delta=10^{-1}$. The test problems are described in Section 1.6. . .

2.1 The L-curve plot of the problem "shaw" $(\mathrm{n}=100)$ where the noise level

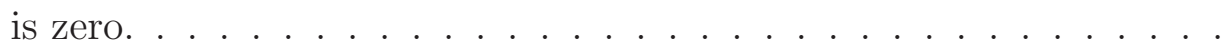

2.2 The L-curve plot of the problem "foxgood" $(\mathrm{n}=100)$ where the noise level is zero. The value of $\alpha$ at corner is $3.0809 \times 10^{-15} \ldots \ldots$. . .

2.3 The L-curve of the problem "heat" $(n=100)$ for $\delta=10^{-1} \ldots \ldots$

3.1 The exact data and the noisy data for the test problem "shaw" $(\mathrm{n}=100)$, with relative error $10^{-1}$ in the noisy data. . . . . . .

3.2 Tikhonov regularization: The exact solution for the "shaw" problem, together with the regularized solutions produced by the L-curve method and multiplicative regularization. In this example, the results of the two methods are essentially the same. . . . . . . . . 
3.3 Tikhonov regularization: The exact solution for the "heat" problem, together with the regularized solutions produced by the L-curve method and multiplicative regularization. In this example, the two methods produce similar estimates, but the L-curve estimate is slightly

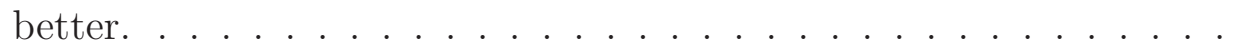

3.4 The exact solution for the "foxgood" problem, together with the regularized solutions produced by the L-curve method and multiplicative regularization. In this example, the two methods produce similar estimates, but multiplicative regularization estimate is slightly better. .

3.5 The test problem "foxgood", with the noise level $10^{-2}$ on the left and the test problem "heat", with the noise level $10^{-3}$ on the right. Both methods perform well. . . . . . . . . . . . . . . .

3.6 The test problem "i_laplace", with the noise level $10^{-4}$ on the left and the test problem "shaw", with the noise level $10^{-5}$ on the right. Both methods perform well. . . . . . . . . . . . . . .

3.7 The test problem "deriv2" with relative error $\delta=10^{-1}$ in the noisy data. The curvature has a well-defined corner but multiplicative regularization fails. The graph on the right is the graph of the L-curve.

3.8 The test problem "phillips" with the noise level $\delta=10^{-1}$ in the noisy data. The curvature has a well-defined corner but multiplicative regularization fails. The graph on the right is the graph of the L-curve. 
3.9 The test problem "deriv2" with relative error $\delta=10^{-5}$ in the noisy data. Both methods fail to identify nonzero regularization parameters. The graph on the right is the graph of the L-curve and it does not have a corner. . . . . . . . . . . . . . . . .

3.10 The test problem "phillips" with relative error $\delta=10^{-5}$ in the noisy data. The curvature has a well-defined corner but multiplicative regularization fails. The graph on the right is the graph of the L-curve.

3.11 The re-descretized test problem "deriv2" with relative error $\delta=10^{-1}$ in the noisy data. Both methods identify nonzero regularization parameters. The graph on the right is the graph of the L-curve and it has a well-defined corner. . . . . . . . . . . . . . . . . .

3.12 The re-discretized test problem "phillips" with relative error $\delta=10^{-1}$ in the noisy data. Both methods identify nonzero regularization parameters. The graph on the right is the graph of the L-curve and has a well-defined corner. . . . . . . . . . . . . . .

3.13 The re-discretized test problem "phillips" with relative error $\delta=10^{-5}$ in the noisy data. Both methods identify nonzero regularization pa-

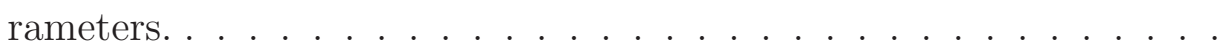


3.14 The re-discretized test problem "deriv2" with relative error $\delta=10^{-5}$ in the noisy data. The multiplicative regularization identifies a nonzero regularization parameter and the L-curve method fails to identify a corner. . . . . . . . . . . . . . . . 


\section{List of Tables}

1.1 The comparison of the singular value expansion and the singular value

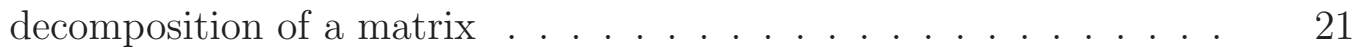

1.2 Summary of test problems . . . . . . . . . . . . . 26

1.3 The degree of ill-posedness of the test problems $\ldots \ldots \ldots \ldots$

1.4 Convergence of the singular values to zero $\ldots \ldots \ldots \ldots$

2.1 The rate of convergence for the L-curve method $\ldots \ldots \ldots$

3.1 The rate of convergence for multiplicative regularization $\ldots \ldots 6$

3.2 Comparison of multiplicative regularization with the L-curve method 68

3.3 Comparison of multiplicative regularization with the L-curve method 69

3.4 Comparison of multiplicative regularization with the L-curve method 71

4.1 Numerical results for the infinite-dimensional test problem "baart" . 94

4.2 Numerical results for the infinite-dimensional test problem "wing" . 95

4.3 Numerical results for the infinite-dimensional test problem "deriv2" 96 



\section{Acknowledgments}

I would like to give my gratitude to my supervisor Dr. Mark S. Gockenbach whose expertise, understanding, and skills, added considerably to my experience. I appreciate his huge patience and deep knowledge.

I am so thankful to the members of my committee, Dr. Jiguang Sun, Dr. Tamara Olson and Dr. Seyedmohsen Azizi for the time and effort they put, in reading my dissertation and giving great suggestions.

I wish to express my sincere thanks to my teaching mentors, Ann M. Humes and Elizabeth J. Reed, for providing me with all the necessary training and support.

I must also acknowledge my wonderful husband, Dr. Foad Yousef, for his unceasing encouragement, support and attention throughout this venture. 



\section{Abstract}

Inverse problems arise in many branches of science and engineering. In order to get a good approximation of the solution of this kind of problems, the use of regularization methods is required. Tikhonov regularization is one of the most popular methods for estimating the solutions of inverse problems. This method needs a regularization parameter and the quality of the approximate solution depends on how good the regularization parameter is.

The L-curve method is a convenient parameter choice strategy for selecting the Tikhonov regularization parameter and it works well most of the time. There are some problems in which the L-curve criterion does not perform properly.

Multiplicative regularization is a method for solving inverse problems and does not require any parameter selection strategies. However, it turns out that there is a close connection between multiplicative regularization and Tikhonov regularization; in fact, multiplicative regularization can be regarded as defining a parameter choice rule for Tikhonov regularization.

In this work, we have analyzed multiplicative regularization for finite-dimensional 
problems. We also have presented some preliminary theoretical results for infinitedimensional problems. Furthermore, we have demonstrated with numerical experiments that the multiplicative regularization method produces a solution that is usually very similar to the solution obtained by the L-curve method. This method is guaranteed to define a positive regularization parameter under some conditions. Computationally, this method is not expensive and is easier to analyze compared to the L-curve method. 


\section{Chapter 1}

\section{Introduction}

\section{$1.1 \quad$ Linear inverse problems}

Let $X$ and $Y$ be Hilbert spaces (that is, complete inner product spaces) and let $T: X \rightarrow Y$ be a linear operator. We will study inverse problems of the following form: given $y \in Y$, find $x \in X$ satisfying $T x=y$.

An equation $T x=y$ in which the operator $T$ has certain properties represents an inverse problem. As we will see, an inverse problem is a certain kind of ill-posed problem. 
The results in this section are well-known; to learn more, see [12] for further explanations.

\subsubsection{Well-posed and ill-posed problems}

Hadamard's definition of a well-posed problem says that a mathematical problem is well-posed if and only if the following properties hold:

1. Existence: A solution exists for all admissible data.

2. Uniqueness: The solution is unique for all admissible data.

3. Stability: The solution depends continuously on data.

If the problem is not well-posed, then we call it ill-posed. If one of the first two properties, existence or uniqueness, fails to hold, the problem is ill-posed, but we do not classify it as an inverse problem. The important property makes the equation an inverse problem is that the solution $x$ does not depend continuously on data $y$ (that is, we have an inverse problem if stability fails to hold). The continuity depends on the norms chosen for both spaces. A given problem can be stable under one norm and unstable under a different norm, but normally we have to work with the given norms on $X$ and $Y$. 
For the problem $T x=y$, existence and uniqueness are equivalent to the existence of $T^{-1}$, and stability is equivalent to the continuity (boundedness) of $T^{-1}$. So $T x=y$ is well-posed if and only if $T^{-1}$ exists and is bounded.

\subsubsection{Dealing with lack of existence and lack of uniqueness}

If a problem is ill-posed because existence or uniqueness fails, then there are standard methods for defining a related problem that may be well-posed.

Lack of existence: If the existence property fails for $T x=y$, it just means that $R(T)$ is not all of $Y$. In this case, for $y \notin R(T)$, we consider the least-squares problem instead: $\min \|T x-y\|$ for $x \in X$.

Definition 1. Let $T: X \rightarrow Y$ be a linear operator. If $x \in X$ satisfies

$$
\|T x-y\|=\inf \{\|T z-y\| \mid z \in X\},
$$

then $x$ is called a least-squares solution of $T x=y$.

For a given $y$, the problem $T x=y$ has a least-squares solution if and only if $y \in$ $R(T) \oplus R(T)^{\perp}$. Also $T x=y$ has a least-squares solution for all $y \in Y$ if and only if $R(T)$ is closed, because when $R(T)$ is closed, $R(T) \oplus R(T)^{\perp}=Y$. 
Recall that the adjoint operator $T^{*}$ is defined by $\langle T x, y\rangle_{Y}=\left\langle x, T^{*} y\right\rangle_{X}$ for all $x \in X$ and $y \in Y$.

Theorem 2. Let $y \in R(T) \oplus R(T)^{\perp}$. Then $\bar{x}$ is a least-squares solution of $T x=y$ if and only if $T^{*} T \bar{x}=T^{*} y$.

Lack of uniqueness: If the uniqueness property fails (but existence holds), that is, if the problem $T x=y$ has more than one solution, we pick the solution of smallest norm and we reformulate the problem as min $\|x\|_{X}$ such that $x$ is a solution of problem $T x=y$. Because $T$ is linear, uniqueness is related to $N(T)$. To be precise, if $T x=y$ has multiple solutions, then $N(T)$ is nontrivial. Conversely, if $N(T)$ is nontrivial and $T x=y$ has a solution, then this solution is not unique.

If $\hat{x}$ is a solution of $T x=y$ and $N(T)$ is nontrivial, then the set of all solutions of $T x=y$ is given by

$$
\hat{x}+N(T)=\{\hat{x}+u \mid u \in N(T)\} .
$$

This set has a unique smallest element, namely, the unique element in $N(T)^{\perp}$. In other words, if $T x=y$ has a solution, then it has a unique solution belonging to $N(T)^{\perp}$, and that is the minimum-norm solution.

If the uniqueness and existence both fail, then we pick the minimum-norm leastsquares solution of the problem $T x=y$.

Definition 3. Let $T: X \rightarrow Y$ be a linear operator. If $x$ is a least-squares solution of 
$T x=y$ and

$$
\|x\|=\inf \{\|z\| \mid z \text { is least-squares solution of } T x=y\},
$$

then $x$ is called the minimum-norm least-squares solution of $T x=y$.

The set of all least-squares solutions of $T x=y$ is given by $\hat{x}+N\left(T^{*} T\right)$, which is the same as $\hat{x}+N(T)$ because $N\left(T^{*} T\right)=N(T)$. The set $\hat{x}+N(T)$ has a unique element in $N(T)^{\perp}$, which is the minimum-norm least-squares solution.

\subsubsection{The Moore-Penrose generalized inverse}

Recall that $T x=y$ is well-posed if and only if $T^{-1}$ exists and is bounded. As we discussed, if existence or uniqueness fails, we try to find the minimum-norm leastsquares solution instead.

We have seen that for each $y \in R(T) \oplus R(T)^{\perp}$, there exists a unique minimumnorm least-squares solution of $T x=y$. We define $T^{\dagger}: R(T) \oplus R(T)^{\perp} \rightarrow X$ by the condition $T^{\dagger} y$ is the minimum-norm least-squares solution of $T x=y$. We write $D\left(T^{\dagger}\right)=R(T) \oplus R(T)^{\perp}$. The operator $T^{\dagger}$ is called the Moore-Penrose generalized inverse of $T$. 
If existence or uniqueness fails (i.e., if our problem has no solution or it has more than one solution), then for $y \in D\left(T^{\dagger}\right)$, the best approximate solution is given by $x=T^{\dagger} y$.

We now see that $\bar{x}=T^{\dagger} y$ is characterized by the condition $T^{*} T \bar{x}=T^{*} y$ and $\bar{x} \in$ $N(T)^{\perp}$. It follows that $R\left(T^{\dagger}\right) \subset N(T)^{\perp}$. Also if we define $\hat{T}=\left.T\right|_{N\left(T^{\perp}\right)}: N(T)^{\perp} \rightarrow$ $R(T)$, since $N(\hat{T})$ is trivial and $R(\hat{T})=R(T)$, then $\hat{T}^{-1}$ exists. Now for all $x \in$ $N(T)^{\perp}, T^{\dagger} T x=\hat{T}^{-1} \hat{T} x=x$, which implies $N(T)^{\perp} \subset R\left(T^{\dagger}\right)$. Therefore $R\left(T^{\dagger}\right)=$ $N(T)^{\perp}$.

Since $R(T)$ is dense in $\overline{R(T)}$, then $D\left(T^{\dagger}\right)$ is dense in $\overline{R(T)} \oplus R(T)^{\perp}=Y$, and thus $T^{\dagger}$ is a densely defined operator. Also $T^{\dagger}$ is a closed operator, i.e., for $y_{n} \subset D\left(T^{\dagger}\right)$, $y_{n} \rightarrow y$ and $T^{\dagger} y_{n} \rightarrow x \in X$ imply that $y \in D\left(T^{\dagger}\right)$ and $x=T^{\dagger} y$. If $R(T)$ is closed, then clearly $D\left(T^{\dagger}\right)=Y$ and $T^{\dagger}: Y \rightarrow X$; therefore by the closed graph theorem, $T^{\dagger}$ is bounded. Also if $T^{\dagger}: D\left(T^{\dagger}\right) \rightarrow X$ and $T^{\dagger}$ is bounded, then by Theorem 2.15 of [12], $D\left(T^{\dagger}\right)$ is closed and as a result $R(T)$ is closed. Now we have the following fundamental result.

Corollary 4. Let $T: X \rightarrow Y$ be a linear operator. Then $T^{\dagger}$ is bounded (i.e., continuous) if and only if $R(T)$ is closed. 


\subsubsection{Compact operators}

Compact operators are central to the study of the inverse problems. Except for certain degenerate cases, every compact operator $K$ defines an inverse problem $K x=y$.

Definition 5. Let $K: X \rightarrow Y$ be linear. Then $K$ is a compact operator if and only if it maps bounded sets in $X$ to pre-compact sets in $Y$. Equivalently $K$ is a compact operator if and only if

$$
\left\{x_{n}\right\} \subset X,\left\{x_{n}\right\} \text { bounded } \Rightarrow\left\{K x_{n}\right\} \text { has a convergent subsequence. }
$$

If $K: X \rightarrow Y$ is a compact operator, then clearly $K$ is bounded because if it is not, then there exists $\left\{x_{n}\right\} \subset X$ with $\left\|x_{n}\right\|_{X}=1$ for all $n$ such that $\left\|K x_{n}\right\| \rightarrow \infty$ as $n \rightarrow \infty$. That implies $\left\{K x_{n}\right\}$ cannot have a convergent subsequence, which is a contradiction.

Here is the degenerate case mentioned above.

Definition 6. Let $K: X \rightarrow Y$ be linear. Then $K$ is a finite-rank operator if and only if $R(K)$ is finite-dimensional.

A finite-rank operator is a type of compact operator because every bounded subset of a finite-dimensional space is pre-compact. 
Theorem 7. Let $K: X \rightarrow Y$ be bounded and finite-rank. Then $K$ is compact.

Theorem 8. Let $K: X \rightarrow Y$ be compact but not finite-rank. Then $R(K)$ is not closed.

The above theorem can be proved by way of contradiction. If we assume $R(K)$ is closed, then it is complete, so the new operator $\hat{K}=\left.K\right|_{N(K)^{\perp}}: N(K)^{\perp} \rightarrow R(K)$ is continuously invertible. Then $K \hat{K}^{-1}=I_{R(K)}$ is compact and hence $R(K)$ is finitedimensional.

\subsubsection{The singular value expansion (SVE)}

A compact operator can always be expressed as a simple form known as the singular value expansion.

Definition 9. Let $X$ and $Y$ be Hilbert spaces and let $x \in X$ and $y \in Y$. The outer product $y \otimes x: X \rightarrow Y$ is the linear operator defined by

$$
(y \otimes x) u=\langle x, u\rangle y \quad \forall u \in X .
$$

Theorem 10. Let $K: X \rightarrow Y$ be compact. Then there exist orthonormal sequences $\left\{\varphi_{n}\right\} \subset X$ and $\left\{\psi_{n}\right\} \subset Y$ and a sequence $\left\{\sigma_{n}\right\}$ of positive numbers such that $\sigma_{1} \geq$ 
$\sigma_{2} \geq \sigma_{3} \geq \ldots>0$, and

$$
K=\sum_{n} \sigma_{n} \psi_{n} \otimes \varphi_{n}
$$

The representation $K=\sum_{n} \sigma_{n} \psi_{n} \otimes \varphi_{n}$ is called the singular value expansion of $K$. If a compact operator $K$ has infinitely many singular values $\sigma_{1}, \sigma_{2}, \ldots$, then $R(K)$ is non-closed, and also $\lim _{n \rightarrow \infty} \sigma_{n}=0$.

Theorem 11. Let $K: X \rightarrow Y$ be a compact linear operator and let $\sum_{n} \sigma_{n} \psi_{n} \otimes \varphi_{n}$ be the SVE of $K$. Then $\left\{\sigma_{n}^{2}\right\}_{\{n \in N\}}$ is the set of nonzero eigenvalues of the self-adjoint operator $K^{*} K$. Also $\left\{\varphi_{n}\right\}_{\{n \in N\}}$ is a set of eigenvectors of $K^{*} K$ and the $\psi_{n}$ is defined by $\psi_{n}=\frac{K \varphi_{n}}{\sigma_{n}}$.

Each $\varphi_{n}$ is an eigenvector of $K^{*} K$ corresponding to $\sigma_{n}^{2}$, and $\left\{\varphi_{n}\right\}$ is a complete orthonormal system for $N(K)^{\perp}$. The following formulas are satisfied:

$$
\begin{gathered}
K \varphi_{n}=\sigma_{n} \psi_{n}, \\
K^{*} \psi_{n}=\sigma_{n} \varphi_{n}, \\
K x=\sum_{n} \sigma_{n}\left\langle x, \varphi_{n}\right\rangle_{X} \psi_{n}, \quad x \in X, \\
K^{*} y=\sum_{n} \sigma_{n}\left\langle y, \psi_{n}\right\rangle_{Y} \varphi_{n}, \quad y \in Y .
\end{gathered}
$$

Theorem 12. Let $\sum_{n} \sigma_{n} \psi_{n} \otimes \varphi_{n}$ be the SVE of the compact linear operator $K$. Then 
we have

1.

$$
y \in D\left(K^{\dagger}\right) \Leftrightarrow \sum_{n} \frac{\left|\left\langle y, \psi_{n}\right\rangle_{Y}\right|^{2}}{\sigma_{n}^{2}}<\infty .
$$

2. For $y \in D\left(K^{\dagger}\right)$,

$$
K^{\dagger} y=\sum_{n} \frac{\left\langle y, \psi_{n}\right\rangle_{Y}}{\sigma_{n}} \varphi_{n}
$$

The first condition is called the Picard condition and it implies that the minimumnorm least-squares solution of $K x=y$ exists only if the Fourier coefficients $\left\langle y, \psi_{n}\right\rangle$ decay faster than the corresponding singular values $\sigma_{n}$. The second condition gives an explicit formula for the minimum-norm least-squares solution $x^{\dagger}=K^{\dagger} y$ of $K x=y$.

\subsubsection{Fredholm integral equations}

A Fredholm integral equation of the first kind is an equation of the form

$$
\int_{0}^{1} k(s, t) f(t) d t=g(s), \quad 0 \leq s \leq 1 .
$$

Here the kernel $k$ and the right-hand-side function $g$ are known functions while the function $f$ is unknown and we would like to know it.

We can view this as a linear operator equation $K f=g$ by defining $K: L^{2}(a, b) \rightarrow$ 
$L^{2}(a, b)$ as

$$
(K f)(s)=\int_{a}^{b} k(s, t) f(t) d t,
$$

where $k \in L^{2}((a, b) \times(a, b))$. Such an operator $K$ is always compact.

Definition 13. Let $K$ be an integral operator of the first kind with kernel $k$. We say that $k$ is degenerate if and only if there exists functions $a_{1}, \ldots, a_{n}, b_{1}, \ldots, b_{n}$ such that

$$
k(x, y)=\sum_{i=1}^{n} a_{i}(x) b_{i}(y) .
$$

It can be shown that $K$ has finite rank if and only if $k$ is degenerate.

\subsection{Tikhonov regularization and parameter choice rules}

Considering an inverse problem where the data $y$ is perturbed by errors, the solution to the perturbed problem is usually dominated by errors (because of the lack of stability - the solution does not depend continuously on the data). In such a case, it is necessary to use a regularization method to compute a solution that is less sensitive to the perturbation.

Tikhonov Regularization is one of the most popular regularization methods for inverse 
problems. It was introduced by Tikhonov [39].

We typically do not know $y^{*}$ exactly but we rather know an estimate $y$ of $y^{*}$. The idea of regularization is to replace the system $T x=y$ with one that is less sensitive to noise in the data. We will now describe the Tikhonov regularization method. (For more information about Tikhonov regularization, and to learn about other regularization methods, see Engl et al. [12] and Hansen [25]).

Tikhonov regularization defines an approximate solution of $T x=y$ as the minimizer of the function

$$
\|T x-y\|_{Y}^{2}+\alpha\|x\|_{X}^{2}
$$

Insisting on making $\|T x-y\|_{Y}^{2}$ as small as possible can lead to $\|x\|_{X}^{2} \rightarrow \infty$, so we prevent this by adding the penalty term $\alpha\|x\|_{X}^{2}$. For each $\alpha>0$, there exists a unique minimizer of (1.2.1):

$$
x_{\alpha, y}=\left(T^{*} T+\alpha I\right)^{-1} T^{*} y .
$$

For the Tikhonov regularization to work, we would expect that for all $y^{*} \in D\left(T^{\dagger}\right)$, $x_{\alpha, y^{*}} \rightarrow T^{\dagger} y^{*}$ as $\alpha \rightarrow 0^{+}$. More generally, since $y^{*}$ is not known exactly, we want $x_{\alpha, y} \rightarrow T^{\dagger} y^{*}$ for $y \notin D\left(T^{\dagger}\right)$ as $(\alpha, y) \rightarrow\left(0^{+}, y^{*}\right)$. We will see that this holds only if $\alpha$ is chosen properly with respect to $y$, specifically, only if $\alpha$ does not converge to zero 
too quickly with respect to $\left\|y-y^{*}\right\|_{Y}$.

These convergence properties must hold for the Tikhonov regularization method. But on a specific problem, we do not have a sequence $\left\{y_{n}\right\}$ converging to $y^{*}$. We just have one measurement $y$ of $y^{*}$, and we need to choose a good value of $\alpha$. The purpose of this dissertation is to study one method for choosing $\alpha$.

A variation of Tikhonov regularization is given by

$$
\|T x-y\|_{Y}^{2}+\alpha\|L x\|_{Z}^{2}
$$

where $L: X \rightarrow Z$. In this case the unique minimizer of the Tikhonov functional is given by

$$
x_{\alpha, y}=\left(T^{*} T+\alpha L^{*} L\right)^{-1} T^{*} y .
$$

We will not study this variation here.

\subsubsection{Definitions and basic properties}

The quality of the approximated solution $x_{\alpha, y}$ depends on choosing a good regularization parameter $\alpha$. Parameter choice method are described by whether they use a

bound $\delta$ on the noise level $\left\|y-y^{*}\right\|_{Y}$. Let $\alpha$ be a regularization parameter choice. Then it is called 
- an a priori parameter choice if it just depends on the noise level $\delta$ : $\alpha=\alpha(\delta)$.

- an a posteriori parameter choice if it depends on both the noise level $\delta$ and the noisy data $y: \alpha=\alpha(\delta, y)$.

- a purely a posteriori (or heuristic) parameter choice if it depends only on the noisy data $y: \alpha=\alpha(y)$.

If we assume $R(T)$ is non-closed, then $T^{\dagger}$ is unbounded. Since in an inverse problem, $T^{\dagger}$ is not bounded and data are not exact, then $T^{\dagger} y$ need not exist and might not be a good approximation for $T^{\dagger} y^{*}$ even if does exist. That is the reason for using regularization methods for approximating $T^{\dagger} y^{*}$. We look for an approximation $x_{\alpha, y}$ of $T^{\dagger} y^{*}$ which depends continuously on the data and has the property that $x_{\alpha, y} \rightarrow T^{\dagger} y^{*}$ as $\delta \rightarrow 0$, provided $\alpha$ is chosen properly. However, based on the result of Bakushinskii [3], this is not possible if the parameter choice rule depends only on the noisy data $(\alpha=\alpha(y))$, unless $T$ has finite rank. In other words, no purely a posteriori parameter choice strategy can yield a convergent regularization method.

The advantage of a purely a posteriori method is that it does not require a knowledge of $\delta$. Since this kind of method can not define a convergent regularization method unless $T$ has finite rank, we consider this kind of method as a heuristic parameter choice rule. 
The following theorem gives the basic convergence results about Tikhonov regularization.

Theorem 14. [12] Let $x_{\alpha, y}$ be the unique minimizer of the Tikhonov functional, $y^{*} \in R(T),\left\|y-y^{*}\right\| \leq \delta$ and let $\alpha=\alpha(\delta)$ be such that

$$
\lim _{\delta \rightarrow 0} \alpha(\delta)=0
$$

and

$$
\lim _{\delta \rightarrow 0} \frac{\delta^{2}}{\alpha(\delta)}=0
$$

Then

$$
\lim _{\delta \rightarrow 0} x_{\alpha, y}=T^{\dagger} y^{*}
$$

The theorem implies that sufficient conditions for strong convergence are $\alpha(\delta) \rightarrow 0$ and $\frac{\delta^{2}}{\alpha(\delta)} \rightarrow 0$, as $\delta \rightarrow 0$.

The condition $\frac{\delta^{2}}{\alpha(\delta)} \rightarrow 0$ implies that $\alpha$ must converge to zero at the slower rate than $\delta^{2}$. If we have $\alpha=C \delta^{p}$ (where $C$ is a constant), then in order to obtain convergence we must have $0<p<2$.

If $\lim _{\delta \rightarrow 0} \alpha(\delta)=0$ and $\frac{\delta^{2}}{\alpha(\delta)}$ is only bounded above, then $\left\|x_{\alpha, y}-T^{\dagger} y^{*}\right\|_{X}^{2} \leq C$ and $x_{\alpha, y} \rightarrow T^{\dagger} y^{*}$ weakly. 


\subsection{Multiplicative regularization}

The parameter selection is the most difficult part in the regularization of an inverse problem, and finding a proper Tikhonov regularization parameter is not always straightforward. To avoid the difficulties of choosing an proper regularization parameter, Berg, Broekhoven and Abubaker [41] and [2] proposed a new regularization method.

Let $T x=y$ be a linear inverse problem, where $T: X \rightarrow Y, y \in Y$ is given, and $x$ is to be estimated. Multiplicative regularization tries to estimate $x$ by solving

$$
\min _{x \in X} J(x ; y)
$$

where $J(x ; y)=\frac{1}{2}\|T x-y\|_{Y}^{2}\|x\|_{X}^{2}$. The goal is to identify a value of $\mathrm{x}$ that makes the residual $\|T x-y\|_{Y}^{2}$ small without letting the regularized solution $\|x\|_{X}^{2}$ to be large. Note that we must ignore the global minimizer $x=0$ and calculate a local minimizer (if one exists) near $T^{\dagger} y^{*}$.

To analyze this problem, we notice that

$$
\nabla_{x} J(x, y)=\|T x-y\|_{Y}^{2} x+\|x\|_{X}^{2}\left(T^{*} T x-T^{*} y\right)
$$


If $x$ is a minimizer of $J$, then $\nabla_{x} J(x, y)=0$. Assuming $x \neq 0, \nabla_{x} J(x, y)=0$ is equivalent to

$$
\|T x-y\|_{Y}^{2} x+\|x\|_{X}^{2}\left(T^{*} T x-T^{*} y\right)=0 \Leftrightarrow T^{*} T x-T^{*} y+\frac{\|T x-y\|_{Y}^{2}}{\|x\|_{X}^{2}} x=0 .
$$

Thus $x \neq 0$ is a minimizer of $J$ only if

$$
T^{*} T x+\alpha x=T^{*} y
$$

where

$$
\alpha=\frac{\|T x-y\|_{Y}^{2}}{\|x\|_{X}^{2}}
$$

If $\alpha$ satisfies (1.3.2) and (1.3.3), then $x_{\alpha, y}=\left(T^{*} T+\alpha I\right)^{-1} T^{*} y$ is a stationary point of $J$. Conversely, if $x$ is a nontrivial stationary point of $J$, then $x$ must equal $x_{\alpha, y}$ for $\alpha=\frac{\|T x-y\|_{Y}^{2}}{\|x\|_{X}^{2}}$.

In this dissertation we study multiplicative regularization as a purely a posteriori parameter choice method for Tikhonov regularization. We will evaluate the performance of multiplicative regularization by comparing it with the performance of the L-curve method. 


\subsection{The L-curve criterion}

\subsubsection{Definitions of the L-curve}

The L-curve criterion is a method for choosing the regularization parameter for Tikhonov regularization method. This method is a purely a-posteriori choice method and it works well most of the time, although there exists some problems in which the L-curve criterion does not perform properly (see [21] and [24]).

The linear L-curve is a set of all points $\left(\left\|T x_{\alpha, y}-y\right\|_{Y},\left\|x_{\alpha, y}\right\|_{X}\right)$ such that $\alpha>0$. For the noisy data $y \notin D\left(T^{\dagger}\right)$, the solution norm and residual norm have the following behaviors:

$$
\alpha \rightarrow 0^{+} \Rightarrow\left\{\begin{array}{l}
\left\|x_{\alpha, y}\right\|_{X} \rightarrow \infty \\
\left\|T x_{\alpha, y}-y\right\|_{Y} \rightarrow\left\|P_{\overline{R(K)}} y-y\right\|_{Y}
\end{array}\right.
$$

On the other hand,

$$
\alpha \rightarrow \infty \Rightarrow\left\{\begin{array}{l}
\left\|x_{\alpha, y}\right\|_{X} \rightarrow 0 \\
\left\|T x_{\alpha, y}-y\right\|_{Y} \rightarrow\|y\|_{Y}
\end{array}\right.
$$

Finding a good regularization parameter balances the norm of the residual $\| T x_{\alpha, y}-$ $y \|_{Y}$ and the norm of the regularized solution $\left\|x_{\alpha, y}\right\|_{X}$. 
The $\log$-log L-curve is a set of all points $\left(\log \left\|T x_{\alpha, y}-y\right\|_{Y}^{2}, \log \left\|x_{\alpha, y}\right\|_{X}^{2}\right)$ such that $\alpha>0$.

A graph of $\left\|x_{\alpha, y}\right\|_{X}$ versus $\left\|T x_{\alpha, y}-y\right\|_{Y}$ tends to have a distinctive shape, especially when plotted in log-log scale. This curve frequently has an L-shaped appearance with an obvious corner separating the vertical and the horizontal parts (see [24]). Using the $\log$-log plot can be useful to increase the sharpness of the corner. The L-curve method chooses the regularization parameter $\alpha$ by picking the value that corresponds to the corner of the L-curve, which is defined as the point of maximum curvature.

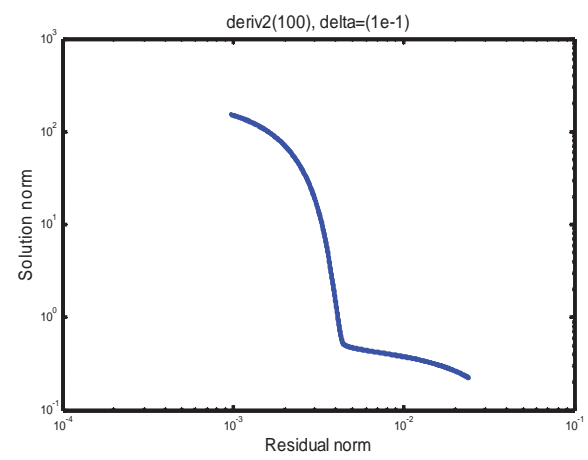

Figure 1.1: The L-curve plot of the test problem "deriv2" $(\mathrm{n}=100)$ where the noise level is $\delta=10^{-1}$. The test problems are described in Section 1.6. 


\subsection{Regularization and finite-dimensional prob- lems}

Finite dimensional problems can not be unstable. However, if $A x=b$ is obtained by discretizing an inverse problem $T x=y$, then $A$ is generally very ill-conditioned. An ill-conditioned system $A x=b$ has essentially the same character as inverse problem $T x=y$ : the solution is very sensitive to errors in the data.

\subsubsection{The singular value decomposition (SVD)}

The singular value decomposition is a valuable tool for dealing with problems with ill-conditioned matrices. Let $A \in R^{m \times n}$ be a rectangular matrix where $m \geq n$. Then the singular value decomposition of $A$ can be defined as $A=U \Sigma V^{T}=\sum_{i=1}^{n} \sigma_{i} u_{i} v_{i}^{t}$, where $U=\left(u_{1}, \ldots, u_{m}\right) \in R^{m \times m}$ and $V=\left(v_{1}, \ldots, v_{n}\right) \in R^{n \times n}$ are orthogonal matrices and $\Sigma=\operatorname{diag}\left(\sigma_{1}, \ldots, \sigma_{n}\right)$ has nonnegative elements such that numbers $\sigma_{1}, \sigma_{2}, \ldots, \sigma_{n}$ are the singular values of $A$, arranged in non-increasing order $\sigma_{1} \geq \sigma_{2} \geq \ldots \geq \sigma_{n} \geq 0$. Every matrix $A \in R^{m \times n}$ has a singular value decomposition.

The minimum-norm least-squares solution of $A x=b$ is given by 


$$
A^{\dagger} b=\sum_{i=1}^{r} \frac{u_{i} \cdot b}{\sigma_{i}} v_{i}
$$

where $r=\operatorname{rank}(A)$.

\subsubsection{The comparison between SVE and SVD}

In the table below, we compare the singular value expansion of an operator $K$ with the singular value decomposition of a matrix $A$.

Table 1.1

The comparison of the singular value expansion and the singular value decomposition of a matrix

\begin{tabular}{|c|c|}
\hline SVE & SVD \\
\hline$\sigma_{1} \geq \sigma_{2} \geq \ldots>0$ & $\sigma_{1} \geq \sigma_{2} \geq \ldots \geq 0$ \\
\hline$\left\langle\varphi_{i}, \varphi_{j}\right\rangle_{X}=\delta_{i, j} \quad i, j=1,2, \ldots$ & $v_{i} \cdot v_{j}=\delta_{i, j} \quad i, j=1, \ldots, n$ \\
\hline$\left\langle\psi_{i}, \psi_{j}\right\rangle_{Y}=\delta_{i, j} \quad i, j=1,2, \ldots$ & $u_{i} \cdot u_{j}=\delta_{i, j} \quad i, j=1, \ldots, n$ \\
\hline$K \varphi_{i}=\sigma_{i} \psi_{i}$ & $A v_{i}=\sigma_{i} u_{i}$ \\
\hline$K^{*} \psi_{i}=\sigma_{i} \varphi_{i}$ & $A^{T} u_{i}=\sigma_{i} v_{i}$ \\
\hline
\end{tabular}

\subsubsection{Tikhonov regularization method}

Let $A x=b$ be an ill-conditioned linear system, where $A \in R^{m \times n}$ and $b \in R^{m}$ are given and $x$ is supposed to be estimated. 
Tikhonov regularization defines an approximate solution of $A x=b$ as the minimizer of the function

$$
\|A x-b\|^{2}+\alpha\|x\|^{2}
$$

The Tikhonov regularized solution is

$$
x_{\alpha, b}=\left(A^{T} A+\alpha I\right)^{-1} A^{*} b .
$$

In terms of the SVD, the regularized solution is given by

$$
x_{\alpha, b}=\sum_{i=1}^{n} \frac{\sigma_{i}^{2}}{\sigma_{i}^{2}+\alpha} \frac{\left(u_{i} \cdot b\right)}{\sigma_{i}} v_{i} .
$$

We call $\frac{\sigma_{i}^{2}}{\sigma_{i}^{2}+\alpha}, i=1,2, \ldots, n$, the filter factors for Tikhonov regularization method and we use them in order to decrease the effect of small singular values. They filter out the contributions of the smaller singular values to the solution, while leaving the effect of the large singular values almost unchanged. This is true because if $\sigma_{i} \gg \alpha$ then $\frac{\sigma_{i}}{\sigma_{i}^{2}+\alpha} \approx \frac{\sigma_{i}}{\sigma_{i}^{2}}=\frac{1}{\sigma_{i}}$, while if $\sigma_{i} \ll \alpha$ then $\frac{\sigma_{i}}{\sigma_{i}^{2}+\alpha} \approx \frac{\sigma_{i}}{\alpha}=0$.

The filter factors for Tikhonov regularization filter out the singular values which are smaller than $\alpha$. Since for the least-squares solution, the largest perturbations are associated with the smallest $\sigma_{i}$, then it is obvious that the regularized solution is less sensitive to the perturbations than the least-squares solution. 


\subsection{Test problems}

We will use a collection of test problems from Hansen's Regularization Tools package [22]. Here are the problems we will use, with a short name for each:

baart: Discretization of the first-kind Fredholm integral equation

$$
\int_{0}^{\pi} k(s, t) f(t) d t=g(s), \quad 0 \leq s \leq \frac{\pi}{2}, 0 \leq t \leq \pi
$$

where $k(s, t)=\exp (s \cos (t)), g(s)=2\left(\frac{\sinh (s)}{s}\right)$ and the solution is $f(t)=\sin (t)$. The equation is discretized by the Galerkin method with orthonormal box functions.

heat: Discretization of the first-kind Volterra integral equation

$$
\int_{0}^{1} k(s, t) f(t) d t=g(s), \quad 0 \leq t, s \leq 1
$$

where $k(s, t)=\frac{t^{\frac{-3}{2}}}{2 \sqrt{\pi}} \exp \left(\frac{-1}{4 t}\right)$, The equation is discretized by means of simple quadrature (midpoint rule). An exact solution is constructed, and then the right-hand side is produced as $b=A x$. 
foxgood: Discretization of the first-kind Fredholm integral equation

$$
\int_{0}^{1} k(s, t) f(t) d t=g(s), \quad 0 \leq t, s \leq 1,
$$

where $k(s, t)=\sqrt{s^{2}+t^{2}}$, and $g(s)=\frac{\left(1+s^{2}\right)^{\frac{3}{2}}-s^{3}}{3}$. The exact solution is $f(t)=t$. The equation is discretized by simple quadrature (midpoint rule).

shaw: Discretization of the first-kind Fredholm integral equation

$$
\int_{\frac{-\pi}{2}}^{\frac{\pi}{2}} k(s, t) f(t) d t=g(s), \quad \frac{-\pi}{2} \leq t, s \leq \frac{\pi}{2}
$$

where $k(s, t)=(\cos (s)+\cos (t))^{2}\left(\frac{\sin (\pi(\sin (s)+\sin (t)))}{\sin (s)+\sin (t)}\right)^{2}$. The exact solution is

$$
f(t)=2 \exp \left(-6(t-0.8)^{2}\right)+\exp \left(-2(t+0.5)^{2}\right),
$$

and right-hand side is produced by $b=A x$. The equation is discretized by simple quadrature.

deriv2: Discretization of the first-kind integral equation

$$
\int_{0}^{1} k(s, t) f(t) d t=g(s), \quad 0 \leq t, s \leq 1,
$$


where

$$
k(s, t)= \begin{cases}s(t-1), & s<t \\ t(s-1), & \text { otherwise }\end{cases}
$$

and $g(s)=\frac{\left(s^{3}-s\right)}{6}$. The exact solution is $f(t)=t$. The equation is discretized by the Galerkin method with orthonormal box functions.

phillips: Discretization of the first-kind integral equation

$$
\int_{0}^{1} k(s, t) f(t) d t=g(s), \quad 0 \leq t, s \leq 1
$$

where $k(s, t)=\phi(s-t)$, and $g(s)=(6-|s|)\left(1+0.5 \cos \left(\frac{\pi s}{3}\right)\right)+\frac{9}{(2 \pi) \sin \left(\frac{\pi|s|}{3}\right)}$. The exact solution is $f(t)=\phi(t)$. The function $\phi$ is defined as

$$
\phi(x)=\left\{\begin{array}{cl}
1+\cos \left(\frac{\pi x}{3}\right), & |x|<3 \\
0, & |x| \geq 3
\end{array}\right.
$$

The equation is discretized by the Galerkin method with orthonormal box functions.

i_laplace: Discretization of the inverse Laplace transformation

$$
\int_{0}^{\infty} k(s, t) f(t) d t=g(s), \quad 0 \leq s, t<\infty
$$

where $k(s, t)=\exp (-s t)$, and $g(s)=\frac{1}{(s+0.5)}$. The exact solution is $f(t)=\exp \left(-\frac{t}{2}\right)$. The equation is discretized by means of Gauss-Laguerre quadrature. 
wing: Discretization of the first-kind Fredholm integral eqaution

$$
\int_{0}^{1} k(s, t) f(t) d t=g(s), \quad 0<t, s<1
$$

where $k(s, t)=t \exp \left(-s t^{2}\right)$, and $g(s)=\frac{\exp \left(-s t_{1}^{2}\right)-\exp \left(-s t_{2}^{2}\right)}{2 s}$ for $t_{1}=\frac{1}{3}$ and $t_{2}=\frac{2}{3}$. The solution $f(t)$ is given by where

$$
f(t)=\left\{\begin{array}{cc}
1, & t_{1}<t<t_{2} \\
0, & \text { otherwise. }
\end{array}\right.
$$

The equation is discretized by the Galerkin method with orthonormal box functions.

Table 1.2

Summary of test problems

\begin{tabular}{|c|l|}
\hline Test problems & \\
\hline deriv2 & Computation of the second derivative, a mildly ill-posed problem \\
\hline phillips & Discretization of a first king Fredholm integral equation \\
\hline heat & Inverse heat problem, discretization of a first king Volterra integral equation \\
\hline ilaplace & Inverse Laplace transformation, discretization of a first king Fredholm integral equation \\
\hline foxgood & Severely ill-conditioned problem, discretized by simple quadrature (midpoint rule) \\
\hline baart & Discretization of a first king Fredholm integral equation \\
\hline shaw & $\begin{array}{l}\text { One dimensional image resolution model, discretization of a first king Fredholm integral } \\
\text { equation }\end{array}$ \\
\hline wing & $\begin{array}{l}\text { Test problem with a discontinuous solution, discretization of a first king Fredholm integral } \\
\text { equation }\end{array}$ \\
\hline
\end{tabular}

\subsubsection{The degree of ill-posedness}

The degree of ill-posedness describes how fast the singular values $\sigma_{n}$ converge to zero as $n \rightarrow \infty$. For each test problem, we found the singular values for $n=100$. If there 
exists a positive real number $q$ such that $\sigma_{n}=c n^{-q}$ (where $c$ is a constant), then the problem is called mildly or moderately ill-posed. If on the other hand we have $\sigma_{n}=c e^{-q n^{t}}$, then the problem is called severely ill-posed. The results are given in Table 1.3. It turns out that "deriv2" and "phillips" are mildly ill-posed problems and the rest of the test problems are severely ill-posed.

Table 1.3

The degree of ill-posedness of the test problems

\begin{tabular}{|c|c|c|}
\hline Test problems & $\sigma_{n}=O\left(n^{-q}\right)$ & $\sigma_{n}=O\left(e^{-q n^{t}}\right)$ \\
\hline deriv2 & $\checkmark$ & \\
\hline phillips & $\checkmark$ & \\
\hline heat & & $\checkmark$ \\
\hline i_laplace & & $\checkmark$ \\
\hline foxgood & & $\checkmark$ \\
\hline baart & & $\checkmark$ \\
\hline shaw & & $\checkmark$ \\
\hline wing & & $\checkmark$ \\
\hline
\end{tabular}

Next, we tried to find the best fit for each test problem and the results are given in Table 1.4.

Table 1.4

Convergence of the singular values to zero

\begin{tabular}{|c|c|c|}
\hline Test problems & $\sigma_{n} \approx c n^{-q}$ & $\sigma_{n} \approx c e^{-q n^{t}}$ \\
\hline deriv2 & $(c, q)=(-0.88706,2.1191)$ & \\
\hline phillips & $(c, q)=(2.8293,3.6590)$ & \\
\hline heat & & $(c, q, t)=(0.45411,0.85161,0.57676)$ \\
\hline i_laplace & & $(c, q, t)=(4.9452,0.63472,1.0893)$ \\
\hline foxgood & & $(c, t, q)=(2.0765,2.5645,0.76484)$ \\
\hline shaw & & $(c, q, t)=(2.9445,0.37951,1.2961)$ \\
\hline baart & & $(c, q, t)=(5.5648,0.66672,1.08300)$ \\
\hline wing & & $(c, q, t)=(2.0295,1.7269,1.3599)$ \\
\hline
\end{tabular}




\subsection{Outline of dissertation}

We have seen that for an inverse problems, it is necessary to use a regularization method to compute a solution that is less sensitive to the noise in the data. Most methods require us to choose a good regularization parameter $\alpha$.

In Chapter 2, we review existing parameter choice rules, especially the L-curve method, which is the most popular purely a posteriori rule.

The L-curve parameter choice strategy is a useful method but sometimes it fails to find a proper regularization parameter. Multiplicative regularization was originally introduced to avoid the need to choose a parameter. However, as we saw in Chapter 1, it can be viewed as a parameter choice method for Tikhonov regularization. We analyze multiplicative regularization for finite-dimensional problems in Chapter 3. Numerical results show that both methods, multiplicative regularization and the Lcurve method, perform remarkably similarly provided $A \in \mathbb{R}^{m \times n}$ and $\operatorname{col}(A)$ is a proper subspace of $\mathbb{R}^{m}$.

In Chapter 4, we present some preliminary theoretical and numerical results on the performance of multiplicative regularization on infinite-dimensional problems. The main results and the numerical experiments are found in Chapter 3 and 4 . 
In Chapter 5, we present our conclusions and discuss the future plans and goal. 



\section{Chapter 2}

\section{Literature review}

\subsection{Multiplicative regularization}

The parameter selection is the most difficult part in the regularization of an illposed problem, and finding a proper Tikhonov regularization parameter is not always straightforward. In order to avoid the difficulties of choosing a proper regularization parameter, Berg, Broekhoven and Abubaker in [41] and [2] proposed a new regularization method. The main advantage of this method is that we do not have to determine the regularization parameter. In this method, no a priori knowledge on the data is needed.

Multiplicative regularization has recently been studied in the $\mathrm{PhD}$ dissertation of 
Orozco Rodríquez [38]. He analyzes the multiplicative regularization as a parameter choice strategy and compares the performance of multiplicative regularization with the L-curve criterion for an image deblurring problem.

Multiplicative regularization has been used in a variety of applications in the past decade (see for example [2] and [1]).

\subsection{Popular parameter choice rules}

Various methods have been introduced in the literature for selecting the regularization parameter $\alpha$. Parameter choice strategies are categorized based on their assumptions about the error norm. Here we introduce the most popular ones briefly.

\subsubsection{Morozov discrepancy principle}

Morozov discrepancy principle is the most popular a-posteriori parameter choice rule for Tikhonov regularization [32]. This method chooses $\alpha$ to satisfy

$$
\left\|T x_{\alpha, y}-y\right\|=\delta
$$


that is, the regularization parameter is chosen via a comparison between the residual and the noise level. The equation (2.2.1) has a solution $\alpha=\alpha(\delta, y)>0$ as long as $\left\|y-y^{*}\right\| \leq \delta,\|y\|>\delta$, and $\left(y-y^{*}\right) \notin R(T)^{\perp}$. The discrepancy principle defines a convergent regularization method provided $y^{*} \in R(T)$.

In the real world examples, the information about the noise level is not always available. Therefore it is necessary to consider some other parameter choice rules that avoid knowledge of noise level. Such a heuristic parameter choice rule is called a purely a posteriori parameter choice method.

\subsubsection{Generalized cross-validation method}

This method was introduced by Golub et al. [40] and it is a purely a posteriori parameter choice method. This method includes statistical considerations and does not require any prior knowledge about the error. The method selects a regularization parameter in order to minimize the predicted data error of the regularized solution.

Although generalized cross-validation is an accurate purely a-posteriori method for choosing the regularization parameter (see [17]), it has been rarely applied to large scale problems. A major difficulty lies in the evaluation of the cross-validation function which requires the calculation of the trace of an inverse matrix (see [14]). 
The next parameter selecting strategy is the L-curve method which is one of the most popular parameter choice methods. We discuss this method in details since we compare the performance of multiplicative regularization with this method.

\subsection{The L-curve method}

The L-curve criterion is a parameter choice strategy for the Tikhonov regularization method. The use of the L-curve goes back to Miller [31]. In infinite dimensions, no convergence result is possible, as explained in Section 1.2. The L-curve method sometimes fails to find a proper regularization parameter, but it still is a useful method.

\subsubsection{The definition of the corner}

A difficulty with the L-curve method is that a wide range of regularization parameters corresponding to points on the L-curve near the corner. Therefore the corner of the curve should be selected by some numerical method rather than by visual selection. Hansen and O'Leary [24] proposed a method for choosing $\alpha$ when the errors of the

right hand side are white noise ${ }^{1}$. They defined the corner of a continuous L-curve

$\overline{{ }^{1} E\left[b-b^{*}\right]=0 \text { and } E\left[\left(b-b^{*}\right) \cdot\left(b-b^{*}\right)\right]}=\sigma^{2} I$ where $E[\cdot]$ denotes the expectation. 
as the point where the curvature attains a local maximum and they presented an algorithm for locating the corner of the L-curve.

\subsubsection{Concavity of the L-curve}

In linear scale, independently of the right hand side, the L-curve is always convex. For finite-dimensional problems, Reginska [36] proved the following lemmas that describe the shape of the L-curve in logarithmic scale.

1. The L-curve in logarithmic scale is always concave for $\alpha$ less than or equal the smallest singular value, i.e., $\left(\alpha \leq \sigma_{n}\right)$ and also for $\alpha$ greater than or equal to the largest singular value, i.e., $\left(\alpha \geq \sigma_{1}\right)$.

2. Let $i_{1}<i_{2}$ be such that for $i \in\left\{i_{1}, i_{2}\right\}$ we have $u_{i}^{T} \cdot b \neq 0$ and also for $i \notin\left\{i_{1}, i_{2}\right\}$ we have $u_{i}^{T} \cdot b=0$. If $\frac{\left|u_{i_{2}} \cdot b\right|}{\sigma_{i_{2}}^{2}}<\frac{\left|u_{i_{1}} \cdot b\right|}{\sigma_{i_{1}}^{2}}$ then the L-curve in logarithmic scale is strictly concave.

The first result ensures that both ends of a L-curve is always concave but it does not

deal with the behavior of the L-curve for $\alpha \in\left[\sigma_{1}, \sigma_{n}\right]$. The second result constructs a class of specific examples for which the L-curve in logarithmic scale does not have a corner; in particular, it proves that the L-curve does not always have a corner.

Hansen [23] dealt with other conditions under which the L-curve in logarithmic scale 


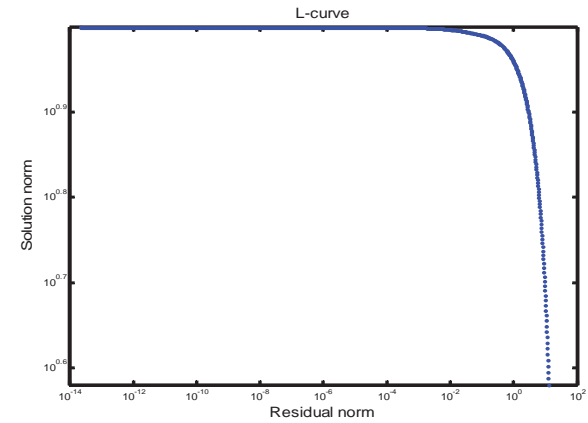

Figure 2.1: The L-curve plot of the problem "shaw" $(n=100)$ where the noise level is zero.

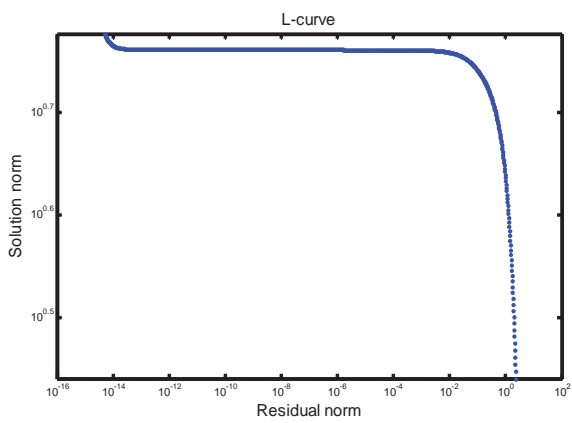

Figure 2.2: The L-curve plot of the problem "foxgood" $(\mathrm{n}=100)$ where the noise level is zero. The value of $\alpha$ at corner is $3.0809 \times 10^{-15}$.

is concave. He gave the heuristic arguments that an exact unperturbed right hand side $b^{*}$ satisfying the discrete Picard condition (see, for example, Figure 2.1) or a right hand side consisting of pure noise e, lead typically to a concave L-curve in logarithmic scale. In certain cases, though, we see other behavior. In certain examples, with $b=b^{*}$, there is a convex corner, though it corresponds to a very small $\alpha$. This happens especially when the matrix $A$ is so severely ill-conditioned that most of the singular values are zero. For instance, the test problem foxgood indicates a convex corner even when $b$ is in the column space of $A$ (see Figure 2.2). 


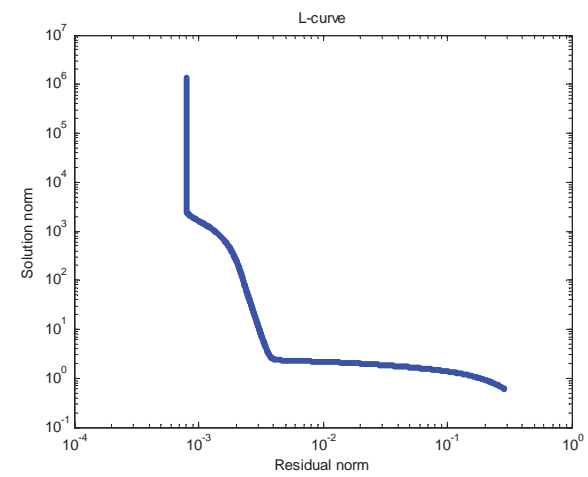

Figure 2.3: The L-curve of the problem "heat" $(n=100)$ for $\delta=10^{-1}$.

Furthermore Bazan [5] did a further study of the convexity properties of the L-curve discussed by Reginska [36] and Hansen [23]. He showed that there exist some situations in which the L-curve criterion fails since the L-curve has several convex corners. The heat problem was chosen in his analysis since this problem most of the time displays more than one convex corner. In this case, Bazan suggested choosing the regularization parameter corresponding to the sharper corner or else to the right-most corner.

\subsubsection{Other disadvantages of the L-curve}

Vogel in [42] studied the behavior of an n-dimensional problem with discrete white noise as $n \rightarrow \infty$, and considered a partially discrete, partially stochastic model $^{2}$ for

\footnotetext{
${ }^{2}$ Let $A_{n}$ is an operator from Hilbert space $Z$ into $R^{n}$. Then the semi-discrete, semi-stochastic data model is given by $\left[b_{n}\right]_{i}=\left[A_{n} x^{*}\right]_{i}+\sigma\left[\eta_{n}\right]_{i}$ for $i=1, \ldots, n$ where $\sigma$ is a fixed operator, $x^{*}$ is the exact solution and it is assumed to be deterministic, and $\eta_{n}$ are discrete white noise vectors with unit covariance which means $E\left(\left[\eta_{n}(x)\right]_{i}\right)=0$ for $i=1, \ldots, n$, and $E\left(\left[\eta_{n}\right]_{i}\left[\eta_{n}\right]_{j}\right)=\delta_{i j}$ for $i, j=1, \ldots, n,(\delta$ is the Dirac delta distribution).
} 
analyzing the L-curve in logarithmic scale. He considered more data samples in his analysis rather than assuming that the noise level in data decreases. He showed that the L-curve method is not convergent under certain conditions. The non-convergence of the L-curve method happens when the solution $T^{\dagger} y^{*}$ is rough or lacks smoothness, that is, when the Fourier coefficients decay more slowly or at the same rate than the singular values. Vogel showed that the regularization parameters computed by this model stagnate, that is, are bounded away from zero.

Another disadvantage of the L-curve is related to very smooth exact solutions. Hanke [16] proved that for this kind of solutions, the L-curve method would fail. He constructed an infinite-dimensional example and showed the chosen regularization parameter by the L-curve method vanishes too rapidly as the noise to signal ratio in the data level goes to zero. Based on the analysis and numerical examples, he showed that the L-curve method may sometimes fail in the sense that the regularized solutions $x_{\alpha, y}$ do not converge to the true solution $x$ of the equation $T x=y$ as $\delta \rightarrow 0$. This can happen when the solution $x$ is very smooth and the noise level is very small.

\subsubsection{Rate of convergence}

If we fit the $\alpha$ computed from the L-curve method to a formula of the form $\alpha=C \delta^{p}$ (where $C$ and $p$ are constant), then we find the following values of $p$ shown in Table 
Table 2.1

The rate of convergence for the L-curve method

\begin{tabular}{|c|c|}
\hline L-curve & \\
\hline Test problem & $\mathrm{p}$ \\
\hline deriv2 & 0.99133 \\
\hline phillips & 1.7481 \\
\hline heat & 2.578 \\
\hline i_laplace & 1.9549 \\
\hline foxgood & 1.9582 \\
\hline shaw & 2.0207 \\
\hline baart & 2.0215 \\
\hline wing & 2.0946 \\
\hline
\end{tabular}

2.1 for the test problems.

\subsection{Conclusions}

The L-curve is a plot of the norm of the regularized solutions versus the norm of the residuals. Under certain conditions, the curve has an L-shaped form with a convex corner; however, this is not always the case. The selected regularization parameter is the point with maximum curvature. The behavior of the L-curve method in some cases is not predictable. The location of the corner depends on the scale of the Lcurve and in some scales, the corner may not appear. Unfortunately, the change of scale does not preserves the convexity of the L-curve. 
Because of difficulties associated with the L-curve method, we will introduce and analyze the multiplicative regularization method as a parameter choice strategy. 


\section{Chapter 3}

\section{Multiplicative regularization for finite-dimensional problems}

In order to avoid the difficulties of choosing a proper regularization parameter, Berg, Broekhoven and Abubaker proposed a new regularization method, which we call multiplicative regularization (see [41], [2]). The method is based on minimizing the function

$$
J(x, b)=\frac{1}{2}\|A x-b\|^{2}\|x\|^{2} .
$$

Multiplicative regularization tries to identify a value of $x$ that makes the residual small without letting the regularized solution be large. As explained in the Introduction, it turns out that there is a close relationship between multiplicative regularization and 
Tikhonov regularization. In fact, multiplicative regularization can be considered as defining a parameter choice method for Tikhonov regularization.

In this chapter, $\|\cdot\|$ denotes the Euclidean norm, and $\langle\cdot, \cdot\rangle$ denotes the Euclidean dot product.

We will study multiplicative regularization for finite-dimensional problems, and we will show by numerical experiments that the performance of this method is comparable to that of the L-curve method.

\subsection{Notation and assumption}

Let $A x=b$ be an ill-conditioned problem, where $A \in \mathbb{R}^{m \times n}$, and $b \in \mathbb{R}^{m}$ are given, and $x$ is to be estimated. We always assume that $b \notin N\left(A^{T}\right)$. We assume that $b^{*}$ is the exact data, $x^{*}$ is the exact solution $\left(A x^{*}=b^{*}, x^{*} \in N(A)^{\perp}\right)$, and $b$ is close to $b^{*}$. We try to find a local minimizer of $J$ that is near $x^{*}$. Notice that $x=0$ is always a global minimizer of $J$, but this is not of interest.

To analyze this problem, we notice that

$$
\nabla_{x} J(x, b)=\|A x-b\|^{2} x+\|x\|^{2}\left(A^{T} A x-A^{T} b\right) .
$$


If $x$ is a minimizer of $J$, then $\nabla_{x} J(x, b)=0$. Assuming $x \neq 0, \nabla_{x} J(x, b)=0$ is equivalent to

$$
\begin{aligned}
\|A x-b\|^{2} x+\|x\|_{X}^{2}\left(A^{T} A x-A^{T} b\right)=0 & \Leftrightarrow A^{T} A x-A^{T} b+\frac{\|A x-b\|^{2}}{\|x\|^{2}} x=0 \\
& \Leftrightarrow A^{T} A x+\frac{\|A x-b\|^{2}}{\|x\|^{2}} x=A^{T} b .
\end{aligned}
$$

Thus $x \neq 0$ is a minimizer of $J$ only if

$$
A^{T} A x+\alpha x=A^{T} b,
$$

where

$$
\alpha=\frac{\|A x-b\|^{2}}{\|x\|^{2}} .
$$

If $\alpha$ satisfies (3.1.1) and (3.1.2), then $x_{\alpha, b}=\left(A^{T} A+\alpha I\right)^{-1} A^{T} b$ is a stationary point of $J$. Conversely, if $x$ is a nontrivial stationary point of $J$, then $x$ must equal $x_{\alpha, b}$ for

$$
\alpha=\frac{\left\|A x_{\alpha, b}-b\right\|^{2}}{\left\|x_{\alpha, b}\right\|^{2}} .
$$

Thus every nontrivial stationary point of $J$ is a Tikhonov solution $x_{\alpha, b}$, where $\alpha$ satisfies (3.1.3).

Reginska [36] has analyzed a similar regularization method by defining the objective function as 


$$
\phi(\alpha, b)=\frac{1}{2}\left\|A x_{\alpha, y}-b\right\|^{2}\left\|x_{\alpha, y}\right\|^{2}
$$

and selecting $\alpha$ by minimizing this function. We can show that $\alpha$ is a stationary point of $\phi(\cdot, b)$ if and only if $\alpha$ satisfies $\alpha=\frac{\left\|A x_{\alpha, y}-b\right\|^{2}}{\left\|x_{\alpha, y}\right\|^{2}}$. To prove this, we first derive some results that will be used repeatedly. We can show that $x_{\alpha, b}$ is a smooth function of $\alpha$ for all $\alpha>0$, and we have the following results:

1. $x_{\alpha, b}^{\prime}=\frac{\partial}{\partial \alpha}\left(x_{\alpha, y}\right)=-N_{\alpha}^{-1} x_{\alpha, b}\left(\right.$ where $\left.N_{\alpha}=\left(A^{T} A+\alpha I\right)\right)$.

2. $\frac{\partial}{\partial \alpha}\left(\left\|x_{\alpha, y}\right\|\right)=2\left\langle x_{\alpha, y}, x_{\alpha, y}^{\prime}\right\rangle$.

3.

$$
\begin{aligned}
\frac{\partial}{\partial \alpha}\left(\left\|A x_{\alpha, y}-b\right\|^{2}\right) & =2\left\langle\left(A x_{\alpha, b}-b\right), A x_{\alpha, b}^{\prime}\right\rangle=2\left\langle\left(A^{T} A x_{\alpha, b}-A^{T} b\right), x_{\alpha, b}^{\prime}\right\rangle \\
& =-2 \alpha\left\langle x_{\alpha, y}, x_{\alpha, y}^{\prime}\right\rangle=-\alpha \frac{\partial}{\partial \alpha}\left(\left\|x_{\alpha, y}\right\|^{2}\right) .
\end{aligned}
$$

(Here we use the fact that $A^{T} A x_{\alpha, b}+\alpha x_{\alpha, b}=A^{T} b$, that is, $A^{T} A x_{\alpha, b}-A^{T} b=$ $\alpha x_{\alpha, b}$

Using the previous results, we have

$$
\begin{aligned}
\phi^{\prime}(\alpha, b) & \left.=-\alpha\left\langle x_{\alpha, b}, x_{\alpha, b}^{\prime}\right\rangle\left\|x_{\alpha, b}\right\|^{2}+2\left\langle x_{\alpha, b}, x_{\alpha, b}^{\prime}\right\rangle\left\|A x_{\alpha, b}-b\right\|^{2}\right) \\
& =\left\langle x_{\alpha, b}, x_{\alpha, b}^{\prime}\right\rangle\left(\left\|A x_{\alpha, b}-b\right\|^{2}-\alpha\left\|x_{\alpha, b}\right\|^{2}\right) .
\end{aligned}
$$


For $b \notin N\left(A^{T}\right),\left\langle x_{\alpha, y}, x_{\alpha, y}^{\prime}\right\rangle$ is never zero. That is true because we have

$$
\left\langle x_{\alpha, y}, x_{\alpha, y}^{\prime}\right\rangle=-\left\langle N_{\alpha}^{-1} A^{T} b, N_{\alpha}^{-2} A^{T} b\right\rangle=-\left\langle A^{T} b, N_{\alpha}^{-3} A^{T} b\right\rangle,
$$

and we know that $N_{\alpha}^{-3}$ is symmetric positive definite. Therefore, $\alpha$ is a stationary point of $\phi(\cdot, b)$ if and only if $\alpha=\frac{\left\|A x_{\alpha, b}-b\right\|^{2}}{\left\|x_{\alpha, b}\right\|^{2}}$. So Reginska's approach is closely related to multiplicative regularization.

\subsection{Preliminaries}

Notice that (3.1.3) is equivalent to $\left\|A x_{\alpha, b}-b\right\|^{2}-\alpha\left\|x_{\alpha, b}\right\|^{2}=0$. We define

$$
F(\alpha, b)=\left\|A x_{\alpha, b}-b\right\|^{2}-\alpha\left\|x_{\alpha, b}\right\|^{2},
$$

for $\alpha>0$ and all $b \in \mathbb{R}^{m}$.

Let $r=\operatorname{rank}(A)$. We will now express the Tikhonov solution of $\mathrm{Ax}=\mathrm{b}$ in terms of the singular value decomposition of A. The reduced SVD of A is

$$
A=\sum_{i=1}^{r} \sigma_{i} \psi_{i} \otimes \varphi_{i}
$$

where $\left\{\varphi_{1}, \varphi_{2}, \ldots, \varphi_{r}\right\}$ is an orthonormal set in $\mathbb{R}^{n},\left\{\psi_{1}, \psi_{2}, \ldots, \psi_{r}\right\}$ is an orthonormal 
set in $\mathbb{R}^{m}, \sigma_{1} \geq \sigma_{2} \geq \ldots \geq \sigma_{r}>0$. It can be show that $A^{T}=\sum_{i=1}^{r} \sigma_{i} \varphi_{i} \otimes \psi_{i}$. If we extend $\left\{\varphi_{1}, \varphi_{2}, \ldots, \varphi_{r}\right\}$ to an orthonormal basis $\left\{\varphi_{1}, \varphi_{2}, \ldots, \varphi_{n}\right\}$ of $\mathbb{R}^{n}$, then $\left\{\varphi_{r+1}, \ldots, \varphi_{n}\right\}$ is a basis for $N(A)$ and every $x \in \mathbb{R}^{n}$ is given by $x=\sum_{i=1}^{n}\left\langle x, \varphi_{i}\right\rangle \varphi_{i}$. We have

$$
\begin{aligned}
A^{T} A x & =A^{T} A\left(\sum_{i=1}^{n}\left\langle x, \varphi_{i}\right\rangle \varphi_{i}\right) \\
& =A^{T}\left(\sum_{i=1}^{r} \sigma_{i}\left\langle x, \varphi_{i}\right\rangle \psi_{i}\right) \\
& =\sum_{i=1}^{r} \sigma_{i}^{2}\left\langle x, \varphi_{i}\right\rangle \varphi_{i},
\end{aligned}
$$

and therefore

$$
\begin{aligned}
\left(A^{T} A+\alpha I\right) x & =\sum_{i=1}^{r} \sigma_{i}^{2}\left\langle x, \varphi_{i}\right\rangle \varphi_{i}+\alpha \sum_{i=1}^{n}\left\langle x, \varphi_{i}\right\rangle \varphi_{i} \\
& =\sum_{i=1}^{r}\left(\sigma_{i}^{2}+\alpha\right)\left\langle x, \varphi_{i}\right\rangle \varphi_{i}+\alpha \sum_{i=r+1}^{n}\left\langle x, \varphi_{i}\right\rangle \varphi_{i} .
\end{aligned}
$$

If we extend $\left\{\psi_{1}, \psi_{2}, \ldots, \psi_{r}\right\}$ to an orthonormal basis $\left\{\psi_{1}, \psi_{2}, \ldots, \psi_{m}\right\}$ of $\mathbb{R}^{m}$, then $\left\{\psi_{r+1}, \ldots, \psi_{m}\right\}$ is a basis for $N\left(A^{T}\right)$ and every $b \in \mathbb{R}^{m}$ is given by $b=\sum_{i=1}^{m}\left\langle b, \psi_{i}\right\rangle \psi_{i}$.

Thus we have

$$
A^{T} b=A^{T}\left(\sum_{i=1}^{m}\left\langle b, \psi_{i}\right\rangle \psi_{i}\right)=\sum_{i=1}^{r} \sigma_{i}\left\langle b, \psi_{i}\right\rangle \varphi_{i}
$$

We know that $\left(A^{T} A+\alpha I\right) x_{\alpha, b}=A^{T} b$ and it follows that 


$$
\begin{aligned}
& \sum_{i=1}^{r}\left(\sigma_{i}^{2}+\alpha\right)\left\langle x_{\alpha, b}, \varphi_{i}\right\rangle \varphi_{i}+\alpha \sum_{i=r+1}^{n}\left\langle x, \varphi_{i}\right\rangle \varphi_{i}=\sum_{i=1}^{r} \sigma_{i}\left\langle b, \psi_{i}\right\rangle \varphi_{i} \\
& \Rightarrow\left\{\begin{array}{cc}
\left(\sigma_{i}^{2}+\alpha\right)\left\langle x_{\alpha, b}, \varphi_{i}\right\rangle=\sigma_{i}\left\langle b, \psi_{i}\right\rangle, & i=1,2, \ldots, r, \\
\alpha\left\langle x_{\alpha, b}, \varphi_{i}\right\rangle=0, & i=r+1, \ldots, n,
\end{array}\right. \\
& \Rightarrow\left\{\begin{array}{cc}
\left\langle x_{\alpha, b}, \varphi_{i}\right\rangle=\frac{\sigma_{i}}{\sigma_{i}^{2}+\alpha}\left\langle b, \psi_{i}\right\rangle, & i=1,2, \ldots, r, \\
\left\langle x_{\alpha, b}, \varphi_{i}\right\rangle=0, & i=r+1, \ldots, n,
\end{array}\right. \\
& \Rightarrow x_{\alpha, b}=\sum_{i=1}^{r} \frac{\sigma_{i}}{\sigma_{i}^{2}+\alpha}\left\langle b, \psi_{i}\right\rangle \varphi_{i} .
\end{aligned}
$$

We then have

$$
\begin{aligned}
A x_{\alpha, b}-b & =A\left(\sum_{i=1}^{r} \frac{\sigma_{i}}{\sigma_{i}^{2}+\alpha}\left\langle b, \psi_{i}\right\rangle \varphi_{i}\right)-\sum_{i=1}^{m}\left\langle b, \psi_{i}\right\rangle \psi_{i} \\
& =\sum_{i=1}^{r} \frac{\sigma_{i}^{2}}{\sigma_{i}^{2}+\alpha}\left\langle b, \psi_{i}\right\rangle \psi_{i}-\sum_{i=1}^{m}\left\langle b, \psi_{i}\right\rangle \psi_{i} \\
& =\sum_{i=1}^{r} \frac{-\alpha}{\sigma_{i}^{2}+\alpha}\left\langle b, \psi_{i}\right\rangle \psi_{i}-\sum_{i=r+1}^{m}\left\langle b, \psi_{i}\right\rangle \psi_{i} .
\end{aligned}
$$

For $\alpha>0$, we have

$$
\begin{aligned}
F(\alpha, b) & =\left\|A x_{\alpha, b}-b\right\|^{2}-\alpha\left\|x_{\alpha, b}\right\|^{2} \\
& =\sum_{i=1}^{r} \frac{\alpha^{2}}{\left(\sigma_{i}^{2}+\alpha\right)^{2}}\left|\left\langle b, \psi_{i}\right\rangle\right|^{2}+\sum_{i=r+1}^{m}\left|\left\langle b, \psi_{i}\right\rangle\right|^{2}-\alpha \sum_{i=1}^{r} \frac{\sigma_{i}^{2}}{\left(\sigma_{i}^{2}+\alpha\right)^{2}}\left|\left\langle b, \psi_{i}\right\rangle\right|^{2} \\
& =\sum_{i=1}^{r} \frac{\alpha^{2}-\alpha \sigma_{i}^{2}}{\left(\sigma_{i}^{2}+\alpha\right)^{2}}\left|\left\langle b, \psi_{i}\right\rangle\right|^{2}+\sum_{i=r+1}^{m}\left|\left\langle b, \psi_{i}\right\rangle\right|^{2}
\end{aligned}
$$


Note that for each $b \in \mathbb{R}^{m}, \lim _{\alpha \rightarrow 0^{+}} x_{\alpha, b}$ exists, and

$$
\lim _{\alpha \rightarrow 0^{+}} x_{\alpha, b}=\lim _{\alpha \rightarrow 0^{+}} \sum_{i=1}^{r} \frac{\sigma_{i}}{\sigma_{i}^{2}+\alpha}\left\langle b, \psi_{i}\right\rangle \varphi_{i}=\sum_{i=1}^{r} \frac{\left\langle b, \psi_{i}\right\rangle}{\sigma_{i}} \varphi_{i}
$$

We define $x_{0, b}=\sum_{i=1}^{r} \frac{\left\langle b, \psi_{i}\right\rangle}{\sigma_{i}} \varphi_{i}$. We will show that $x_{0, b}$ is the minimum-norm leastsquares solution of $A x=b$, that is, that $x_{0, b}$ solves

$$
\begin{aligned}
& \min \|x\| \\
& \text { s.t. } A^{T} A x=A^{T} b .
\end{aligned}
$$

To see this, let $x$ be any least-squares solution of $A x=b$. Then we have

$$
\begin{aligned}
A^{T} A x=A^{T} b & \Rightarrow \sum_{i=1}^{r} \sigma_{i}^{2}\left\langle x, \varphi_{i}\right\rangle \varphi_{i}=\sum_{i=1}^{r} \sigma_{i}\left\langle b, \psi_{i}\right\rangle \varphi_{i} \\
& \Rightarrow \sigma_{i}^{2}\left\langle x, \varphi_{i}\right\rangle=\sigma_{i}\left\langle b, \psi_{i}\right\rangle, \text { for } i=1,2, \ldots, r \\
& \Rightarrow\left\langle x, \varphi_{i}\right\rangle=\frac{1}{\sigma_{i}}\left\langle b, \psi_{i}\right\rangle, \text { for } i=1,2, \ldots, r \\
& \Rightarrow x=\sum_{i=1}^{r} \frac{1}{\sigma_{i}}\left\langle b, \psi_{i}\right\rangle \varphi_{i}+\sum_{i=r+1}^{n} \beta_{i} \varphi_{i} \\
& \Rightarrow x=x_{0, b}+\sum_{i=r+1}^{n} \beta_{i} \varphi_{i}
\end{aligned}
$$

where $\beta_{r+1}, \ldots, \beta_{n}$ can be any real numbers.

Conversely, we can easily show that if $x=\sum_{i=1}^{r} \frac{1}{\sigma_{i}}\left\langle b, \psi_{i}\right\rangle \varphi_{i}+\sum_{i=r+1}^{n} \beta_{i} \varphi_{i}$, then 
$A^{T} A x=A^{T} b$ and $x$ is a least-squares solution. This shows that $x_{0, b}$ is a least-squares solution of $A x=b$.

So we have $\|x\|^{2}=\left\|x_{0, b}\right\|^{2}+\sum_{i=r+1}^{n} \beta_{i}^{2}$, which implies that $\left\|x_{0, b}\right\|^{2} \leq\|x\|^{2}$ for every other least-squares solutions.

We define $A^{\dagger}$ by the condition that $A^{\dagger} b$ is the unique minimum-norm least-squares solution of $A x=b$. The previous result shows that $A^{\dagger} b=\sum_{i=1}^{r} \frac{1}{\sigma_{i}}\left\langle b, \psi_{i}\right\rangle \varphi_{i}$ for all $b \in \mathbb{R}^{m}$, and hence $A^{\dagger}=\sum_{i=1}^{r} \frac{1}{\sigma_{i}} \varphi_{i} \otimes \psi_{i}$.

We can see that for every $b \in \mathbb{R}^{m}, x_{0, b}=A^{\dagger} b$, that is, $\lim _{\alpha \rightarrow 0^{+}} x_{\alpha, b}=A^{\dagger} b$. We also can show that for all $b \in \mathbb{R}^{m}$ and all $\alpha>0,\left\|x_{\alpha, b}\right\| \leq\left\|x_{0, b}\right\|$ and as a result $\left\|N_{\alpha}^{-1} A^{T}\right\| \leq\left\|A^{\dagger}\right\|$. We argue by the way of contradiction. If we assume that there exists $b \in \mathbb{R}^{m}$ and $\alpha>0$ such that $\left\|x_{0, b}\right\|<\left\|x_{\alpha, b}\right\|$, then since for all $x \in \mathbb{R}^{n}$, $\left\|A x_{0, b}-b\right\|^{2} \leq\|A x-b\|^{2}$, it follows that

$$
\left\|A x_{0, b}-b\right\|^{2}+\alpha\left\|x_{0, b}\right\|^{2}<\left\|A x_{\alpha, b}-b\right\|^{2}+\alpha\left\|x_{\alpha, b}\right\|^{2}
$$

which contradicts the definition of $x_{\alpha, b}$ as the unique minimizer of $\|A x-b\|^{2}+\alpha\|x\|^{2}$.

For $b \in \mathbb{R}^{m}$, we write $b=\bar{b}+\hat{b}$ where $\bar{b}=A x_{0, b}=\operatorname{proj}_{\operatorname{col}(\mathrm{A})^{b}} b$ and $\hat{b}=b-A x_{0, b}=$ $\operatorname{proj}_{\operatorname{col}(\mathrm{A})^{\perp}}$. Since $x_{\alpha, b} \rightarrow x_{0, b}$ as $\alpha \rightarrow 0^{+}$, then $\left\|A x_{\alpha, b}-b\right\|^{2} \rightarrow\left\|A x_{0, b}-b\right\|^{2}=\|\hat{b}\|^{2}$ as $\alpha \rightarrow 0^{+}$, and $\alpha\left\|x_{\alpha, b}\right\|^{2} \rightarrow 0$ as $\alpha \rightarrow 0^{+}$. 
Thus we extend the definition of $F$ as follows:

$$
F(\alpha, b)= \begin{cases}\left\|A x_{\alpha, b}-b\right\|^{2}-\alpha\left\|x_{\alpha, b}\right\|^{2}, & \alpha>0 \\ \|\hat{b}\|^{2}, & \alpha=0\end{cases}
$$

In terms of the SVD, we have

$$
F(\alpha, b)= \begin{cases}\sum_{i=1}^{r} \frac{\alpha^{2}-\alpha \sigma_{i}^{2}}{\left(\sigma_{i}^{2}+\alpha\right)^{2}}\left|\left\langle b, \psi_{i}\right\rangle\right|^{2}+\sum_{i=r+1}^{n}\left|\left\langle b, \psi_{i}\right\rangle\right|^{2}, & \alpha>0, \\ \sum_{i=r+1}^{n}\left|\left\langle b, \psi_{i}\right\rangle\right|^{2}, & \alpha=0 .\end{cases}
$$

For the exact data $b^{*} \in \operatorname{col}(A)$, it follows that $F\left(0, b^{*}\right)=0$. We wish to apply the implicit function theorem to show that $F(\alpha, b)=0$ has a locally unique solution $\alpha=\alpha(b)$ for each $b$ sufficiently close to $b^{*}$. To do this, we must extend $F$ from $[0, \infty) \times \mathbb{R}^{m}$ to $\mathbb{R} \times \mathbb{R}^{m}$ and prove that $F$ is $C^{1}$. First we need to show the following preliminary results.

We know that the problem

$$
\min \|A x-b\|^{2}+\alpha\|x\|^{2}
$$

has a unique solution for each $\alpha>0$ and $b \in \mathbb{R}^{m}$. It is clear that the above problem 
is equivalent to

$$
\min \left\|M_{\alpha} x-(b, 0)\right\|^{2},
$$

where $M_{\alpha} x=(A x, \sqrt{\alpha} x)$. Then the normal equation takes the form $M_{\alpha}^{T} M_{\alpha} x=$ $M_{\alpha}^{T}(b, 0)$ and $x_{\alpha, b}$ is a least-squares solution of it since

$$
\begin{aligned}
M_{\alpha}^{T} M_{\alpha} x_{\alpha, b}=M_{\alpha}^{T}(b, 0) & \Leftrightarrow M_{\alpha}^{T}\left(A x_{\alpha, b}, \sqrt{\alpha} x_{\alpha, b}\right)=M_{\alpha}^{T}(b, 0) \\
& \Leftrightarrow A^{T} A x_{\alpha, b}+\alpha x_{\alpha, b}=A^{T} b \\
& \Leftrightarrow\left(A^{T} A+\alpha I\right) x_{\alpha, b}=A^{T} b \\
& \Leftrightarrow x_{\alpha, b}=N_{\alpha}^{-1} A^{T} b .
\end{aligned}
$$

Since $M_{\alpha} x_{\alpha, b}$ is the projection of $(b, 0)$ onto $\operatorname{col}\left(M_{\alpha}\right)$, then by the Pythagorean theorem we have

$$
\left\|M_{\alpha} x_{\alpha, b}\right\|^{2}+\left\|M_{\alpha} x_{\alpha, b}-(b, 0)\right\|^{2}=\|(b, 0)\|^{2}
$$

which is equivalent to

$$
\left\|A x_{\alpha, b}\right\|^{2}+2 \alpha\left\|x_{\alpha, b}\right\|^{2}+\left\|A x_{\alpha, b}-b\right\|^{2}=\|b\|^{2} .
$$

It follows that

- $\left\|A x_{\alpha, b}\right\|^{2} \leq\|b\|^{2} \Rightarrow\left\|A N_{\alpha}^{-1} A^{T} b\right\| \leq\|b\|, \quad \forall b \in \mathbb{R}^{m} \Rightarrow\left\|A N_{\alpha}^{-1} A^{T}\right\| \leq 1$.

- $\left\|b-A x_{\alpha, b}\right\|^{2} \leq\|b\|^{2} \Rightarrow\left\|b-A N_{\alpha}^{-1} A^{T} b\right\|^{2} \leq\|b\|^{2}, \forall b \in \mathbb{R}^{m} \Rightarrow\left\|\left(I-A N_{\alpha}^{-1} A^{T}\right)\right\| \leq 1$. 
- $2 \alpha\left\|x_{\alpha, b}\right\|^{2} \leq\|b\|^{2} \Rightarrow \sqrt{2 \alpha}\left\|N_{\alpha}^{-1} A^{T} b\right\| \leq\|b\|, \forall b \in \mathbb{R}^{m} \Rightarrow\left\|N_{\alpha}^{-1} A^{T}\right\| \leq \frac{1}{\sqrt{2 \alpha}}$.

Also we know that $\left\|N_{\alpha}^{-1} A^{T}\right\| \leq\left\|A^{\dagger}\right\|$, therefore $\left\|N_{\alpha}^{-1} A^{T}\right\| \leq \min \left\{\frac{1}{\sqrt{2 \alpha}},\left\|A^{\dagger}\right\|\right\}$.

We also derive the following important result.

Lemma 15. For all $\alpha \in(0,1],\left\|N_{\alpha}^{-1}\right\| \leq \frac{1}{\alpha}$.

Proof. For $\alpha>0, x \cdot N_{\alpha} x=x \cdot\left(A^{T} A+\alpha I\right) x=\|A x\|^{2}+\alpha\|x\|^{2} \geq \alpha\|x\|^{2}$. Since $N_{\alpha}$ is a bounded and symmetric operator, it follows that $\left\|N_{\alpha}^{-1}\right\| \leq \frac{1}{\alpha}$, that is, $\left\|\alpha N_{\alpha}^{-1}\right\| \leq 1$ (see [13, Theorem 3.2]).

Lemma 16. For each $b \in \mathbb{R}^{m}$, there exists a unique vector $v_{b} \in \operatorname{col}\left(A^{\dagger}\right)=N(A)^{\perp}$ such that $x_{0, b}=A^{T} A v_{b}$, namely, $v_{b}=\sum_{i=1}^{r} \frac{\left\langle b, \psi_{i}\right\rangle}{\sigma_{i}^{3}} \varphi_{i}$.

Proof. Since $N(A)^{\perp}=\operatorname{span}\left\{\varphi_{1}, \ldots, \varphi_{r}\right\}, v_{b} \in N(A)^{\perp}=\operatorname{col}\left(A^{\dagger}\right)$, and we have

$$
A^{T} A v_{b}=A^{T} A\left(\sum_{i=1}^{r} \frac{\left\langle b, \psi_{i}\right\rangle}{\sigma_{i}^{3}} \varphi_{i}\right)=A^{T}\left(\sum_{i=1}^{r} \frac{\left\langle b, \psi_{i}\right\rangle}{\sigma_{i}^{2}} \psi_{i}\right)=\sum_{i=1}^{r} \frac{\left\langle b, \psi_{i}\right\rangle}{\sigma_{i}} \varphi_{i}=x_{0, y} .
$$

Let $\hat{v}_{b}$ be another solution in $N(A)^{\perp}$ such that $x_{0, b}=A^{T} A \hat{v}_{b}$. Then $v_{b}-\hat{v}_{b} \in N(A)^{\perp}$ and $v_{b}-\hat{v}_{b} \in N\left(A^{T} A\right)=N(A)$, therefore $v_{b}-\hat{v}_{b} \in N(A) \cap N(A)^{\perp}=\{0\}$ and the proof is complete. 
Notice that $v_{b}$ is the Lagrange multiplier for the problem

$$
\begin{aligned}
& \min \|x\| \\
& \text { s.t. } A^{T} A x=A^{T} b .
\end{aligned}
$$

This vector will be used later.

\subsection{Analysis of multiplicative regularization}

To apply the desired form of the implicit function theorem, we must show that $F$

is $C^{1}$. First we will show that the $F$ is a continuous function. We start with the following proof.

Lemma 17. Let $b_{0} \in \mathbb{R}^{m}$ be given. Then $x_{\alpha, b} \rightarrow x_{0, b_{0}}$ as $(\alpha, b) \rightarrow\left(0, b_{0}\right)$.

Proof. We have

$$
\begin{aligned}
\lim _{(\alpha, b) \rightarrow\left(0, b_{0}\right)} x_{\alpha, b} & =\lim _{(\alpha, b) \rightarrow\left(0, b_{0}\right)} \sum_{i=1}^{r} \frac{\sigma_{i}}{\sigma_{i}^{2}+\alpha}\left\langle b, \psi_{i}\right\rangle \varphi_{i} \\
& =\lim _{(\alpha, b) \rightarrow\left(0, b_{0}\right)} \sum_{i=1}^{r} \frac{\sigma_{i}}{\sigma_{i}^{2}+\alpha}\left\langle b-b_{0}, \psi_{i}\right\rangle \varphi_{i}+\lim _{\alpha \rightarrow 0} \sum_{i=1}^{r} \frac{\sigma_{i}}{\sigma_{i}^{2}+\alpha}\left\langle b_{0}, \psi_{i}\right\rangle \varphi_{i} \\
& =\sum_{i=1}^{r} \frac{\left\langle b_{0}, \psi_{i}\right\rangle}{\sigma_{i}} \varphi_{i}=x_{0, b_{0}} .
\end{aligned}
$$


Theorem 18. The function $F:[0, \infty) \times \mathbb{R}^{m} \rightarrow \mathbb{R}$ defined by

$$
F(\alpha, b)= \begin{cases}\left\|A x_{\alpha, b}-b\right\|^{2}-\alpha\left\|x_{\alpha, b}\right\|^{2}, & \alpha>0 \\ \|\hat{b}\|^{2} . & \alpha=0\end{cases}
$$

is continuous.

Proof. It is obvious that $F$ is continuous on $(0, \infty) \times \mathbb{R}^{m}$. We must show that, for all $b_{0} \in \mathbb{R}^{m}$,

$$
\lim _{(\alpha, b) \rightarrow\left(0, b_{0}\right)} F(\alpha, b)=F\left(0, b_{0}\right)
$$

We have

$$
\begin{aligned}
\lim _{(\alpha, b) \rightarrow\left(0, b_{0}\right)} F(\alpha, b) & =\lim _{(\alpha, b) \rightarrow\left(0, b_{0}\right)}\left(\sum_{i=1}^{r} \frac{\alpha^{2}-\alpha \sigma_{i}^{2}}{\left(\sigma_{i}^{2}+\alpha\right)^{2}}\left|\left\langle b, \psi_{i}\right\rangle\right|^{2}+\sum_{i=r+1}^{n}\left|\left\langle b, \psi_{i}\right\rangle\right|^{2}\right) \\
& =\lim _{b \rightarrow b_{0}} \sum_{i=r+1}^{n}\left|\left\langle b, \psi_{i}\right\rangle\right|^{2}=\sum_{i=r+1}^{n}\left|\left\langle b_{0}, \psi_{i}\right\rangle\right|^{2}=\left\|\hat{b}_{0}\right\|^{2}=F\left(0, b_{0}\right) .
\end{aligned}
$$

Notice that

$$
\lim _{(\alpha, b) \rightarrow\left(0, b_{0}\right)} \sum_{i=1}^{r} \frac{\alpha^{2}-\alpha \sigma_{i}^{2}}{\left(\sigma_{i}^{2}+\alpha\right)^{2}}\left|\left\langle b, \psi_{i}\right\rangle\right|^{2}=0,
$$

because $\frac{\alpha^{2}-\alpha \sigma_{i}^{2}}{\left(\sigma_{i}^{2}+\alpha\right)^{2}} \rightarrow 0$ as $\alpha \rightarrow 0$, and also $\left\langle b, \psi_{i}\right\rangle \rightarrow\left\langle b_{0}, \psi_{i}\right\rangle$ as $b \rightarrow b_{0}$.

We can show that $x_{\alpha, b}^{\prime} \rightarrow-v_{b}$ as $\alpha \rightarrow 0$. It is clear that $x_{\alpha, b}^{\prime}$, in terms of SVD, is 


$$
x_{\alpha, b}^{\prime}=\frac{\partial}{\partial \alpha}\left(x_{\alpha, b}\right)=\frac{\partial}{\partial \alpha}\left(\sum_{i=1}^{r} \frac{\sigma_{i}}{\sigma_{i}^{2}+\alpha}\left\langle b, \psi_{i}\right\rangle \varphi_{i}\right)=\sum_{i=1}^{r} \frac{-\sigma_{i}}{\left(\sigma_{i}^{2}+\alpha\right)^{2}}\left\langle b, \psi_{i}\right\rangle \varphi_{i} .
$$

Now we can prove the following lemma.

Theorem 19. For all $b \in \mathbb{R}^{m}, x_{\alpha, b}^{\prime} \rightarrow-v_{b}$ as $\alpha \rightarrow 0$.

Proof. We have

$$
\lim _{\alpha \rightarrow 0} x_{\alpha, b}^{\prime}=\lim _{\alpha \rightarrow 0}\left(\sum_{i=1}^{r} \frac{-\sigma_{i}}{\left(\sigma_{i}^{2}+\alpha\right)^{2}}\left\langle b, \psi_{i}\right\rangle \varphi_{i}\right)=-\sum_{i=1}^{r} \frac{\left\langle b, \psi_{i}\right\rangle}{\sigma_{i}^{3}} \varphi_{i}=-v_{b}
$$

and the proof is complete.

Theorem 20. The function $F$ defined by (3.2.1) is $C^{1}$, with

$$
\frac{\partial F}{\partial \alpha}(\alpha, b)= \begin{cases}-4 \alpha x_{\alpha, b} \cdot x_{\alpha, b}^{\prime}-\left\|x_{\alpha, b}\right\|^{2}, & \alpha>0 \\ -\left\|x_{0, b}\right\|^{2}, & \alpha=0\end{cases}
$$

and

$$
\nabla_{b} F(\alpha, b)= \begin{cases}2\left(b-A x_{\alpha, b}+\alpha A x_{\alpha, b}^{\prime}\right), & \alpha>0 \\ 2 \hat{b}, & \alpha=0\end{cases}
$$

Proof. Once again, it is straightforward to show that $F$ is $C^{1}$ for $\alpha>0$. We have 


$$
\begin{aligned}
\frac{\partial F}{\partial \alpha}(\alpha, b) & =\frac{\partial}{\partial \alpha}\left(\left\|A x_{\alpha, b}-b\right\|^{2}-\alpha\left\|x_{\alpha, b}\right\|^{2}\right) \\
& =-2 \alpha\left\langle x_{\alpha, b}, x_{\alpha, b}^{\prime}\right\rangle-2 \alpha\left\langle x_{\alpha, b}, x_{\alpha, b}^{\prime}\right\rangle-\left\|x_{\alpha, b}\right\|^{2} \\
& =-4 \alpha\left\langle x_{\alpha, b}, x_{\alpha, b}^{\prime}\right\rangle-\left\|x_{\alpha, b}\right\|^{2},
\end{aligned}
$$

since $\frac{\partial}{\partial \alpha}\left(\left\|A x_{\alpha, b}-b\right\|^{2}\right)=-\alpha \frac{\partial}{\partial \alpha}\left(\left\|x_{\alpha, b}\right\|^{2}\right)=-2 \alpha\left\langle x_{\alpha, b}, x_{\alpha, b}^{\prime}\right\rangle$. Also, we have

$$
\begin{aligned}
F(\alpha, b) & =\left\langle A x_{\alpha, b}, A x_{\alpha, b}\right\rangle-2\left\langle b, A x_{\alpha, b}\right\rangle+\langle b, b\rangle-\alpha\left\langle x_{\alpha, b}, x_{\alpha, b}\right\rangle \\
& =\left\langle A N_{\alpha}^{-1} A^{T} b, A N_{\alpha}^{-1} A^{T} b\right\rangle-2\left\langle b, A N_{\alpha}^{-1} A^{T} b\right\rangle+\langle b, b\rangle-\alpha\left\langle N_{\alpha}^{-1} A^{T} b, N_{\alpha}^{-1} A^{T} b\right\rangle \\
& =\left\langle b, A N_{\alpha}^{-1} A^{T} A N_{\alpha}^{-1} A^{T} b\right\rangle-2\left\langle b, A N_{\alpha}^{-1} A^{T} b\right\rangle+\langle b, b\rangle-\alpha\left\langle b, A N_{\alpha}^{-2} A^{T} b\right\rangle,
\end{aligned}
$$

and hence

$$
\begin{aligned}
\nabla_{b} F(\alpha, b) & =2 A N_{\alpha}^{-1} A^{T} A x_{\alpha, b}-4 A x_{\alpha, b}+2 b+2 \alpha A x_{\alpha, b}^{\prime} \\
& =2 A\left(I-\alpha N_{\alpha}^{-1}\right) x_{\alpha, b}-4 A x_{\alpha, b}+2 b+2 \alpha A x_{\alpha, b}^{\prime} \\
& =2 A x_{\alpha, b}+2 \alpha A x_{\alpha, b}^{\prime}-4 A x_{\alpha, b}+2 b+2 \alpha A x_{\alpha, b}^{\prime} \\
& =2\left(b-A x_{\alpha, b}+2 \alpha A x_{\alpha, b}^{\prime}\right) .
\end{aligned}
$$

We must show that, for all $b_{0} \in \mathbb{R}^{m}, \frac{\partial F}{\partial \alpha}\left(0, b_{0}\right)$ and $\nabla_{b} F\left(0, b_{0}\right)$ exist and $\frac{\partial F}{\partial \alpha}(\alpha, b) \rightarrow$ $\frac{\partial F}{\partial \alpha}\left(0, b_{0}\right)$ and $\nabla_{b} F(\alpha, b) \rightarrow \nabla_{b} F\left(0, b_{0}\right)$ as $(\alpha, b) \rightarrow\left(0, b_{0}\right)$. We have 


$$
\begin{aligned}
\frac{\partial F}{\partial \alpha}(0, b) & =\lim _{\alpha \rightarrow 0^{+}} \frac{F(\alpha, b)-F(0, b)}{\alpha}=\lim _{\alpha \rightarrow 0^{+}}\left(\sum_{i=1}^{r} \frac{\alpha-\sigma_{i}^{2}}{\left(\sigma_{i}^{2}+\alpha\right)^{2}}\left|\left\langle b, \psi_{i}\right\rangle\right|^{2}\right) \\
& =-\sum_{i=1}^{r} \frac{\left|\left\langle b, \psi_{i}\right\rangle\right|^{2}}{\sigma_{i}^{2}}=-\left\|x_{0, b}\right\|^{2}
\end{aligned}
$$

Also we have

$$
\begin{aligned}
\frac{\partial F}{\partial \alpha}(\alpha, b) & =\frac{\partial}{\partial \alpha}\left(\sum_{i=1}^{r} \frac{\alpha^{2}-\alpha \sigma_{i}^{2}}{\left(\sigma_{i}^{2}+\alpha\right)^{2}}\left|\left\langle b, \psi_{i}\right\rangle\right|^{2}+\sum_{i=r+1}^{n}\left|\left\langle b, \psi_{i}\right\rangle\right|^{2}\right) \\
& =\sum_{i=1}^{r} \frac{3 \alpha \sigma_{i}^{2}-\sigma_{i}^{4}}{\left(\sigma_{i}^{2}+\alpha\right)^{3}}\left|\left\langle b, \psi_{i}\right\rangle\right|^{2},
\end{aligned}
$$

and we see that

$$
\begin{aligned}
\lim _{(\alpha, b) \rightarrow\left(0^{+}, b_{0}\right)} \frac{\partial F}{\partial \alpha}(\alpha, b) & =\lim _{(\alpha, b) \rightarrow\left(0^{+}, b_{0}\right)}\left(\sum_{i=1}^{r} \frac{3 \alpha \sigma_{i}^{2}-\sigma_{i}^{4}}{\left(\sigma_{i}^{2}+\alpha\right)^{3}}\left|\left\langle b, \psi_{i}\right\rangle\right|^{2}\right) \\
& =-\sum_{i=1}^{r} \frac{\left|\left\langle b_{0}, \psi_{i}\right\rangle\right|^{2}}{\sigma_{i}^{2}}=-\left\|x_{0, b_{0}}\right\|^{2}=\frac{\partial F}{\partial \alpha}\left(0, b_{0}\right) .
\end{aligned}
$$

It follows that $\lim _{(\alpha, b) \rightarrow\left(0^{+}, b_{0}\right)} \frac{\partial F}{\partial \alpha}(\alpha, b)=\frac{\partial F}{\partial \alpha}(0, b)$. Also we have $F(0, b)=\|\hat{b}\|^{2}$, and it follows that $\nabla_{b} F(0, b)=2 \hat{b}$. Therefore we have

$$
\begin{aligned}
\lim _{(\alpha, b) \rightarrow\left(0, b_{0}\right)} \nabla_{b} F(\alpha, b) & =\lim _{(\alpha, b) \rightarrow\left(0, b_{0}\right)} 2\left(b-A x_{\alpha, b}+2 \alpha A x_{\alpha, b}^{\prime}\right) \\
& =2\left(b_{0}-A x_{0, b_{0}}\right)=2 \hat{b}_{0}=\nabla_{b} F\left(0, b_{0}\right)
\end{aligned}
$$


The function $F$ is defined for all $\alpha \geq 0$. To apply the implicit function theorem, we must extend $F$ to all of $\mathbb{R} \times \mathbb{R}^{m}$. For a fixed $b$, we define the function $G:[0, \infty) \rightarrow \mathbb{R}$ as $G(\alpha)=F(\alpha, b)$, and shift it vertically to get $\hat{G}(\alpha)=F(\alpha, b)-F(0, b)$. Then $\hat{G}$ satisfies $\hat{G}(0)=0$. Now we form the odd extension of $\hat{G}$ to get

$$
\hat{G}(\alpha)= \begin{cases}\hat{G}(\alpha)=F(\alpha, b)-F(0, b), & \alpha \geq 0 \\ -\hat{G}(-\alpha)=-F(-\alpha, b)+F(0, b), & \alpha<0\end{cases}
$$

Finally we shift the function $\hat{G}(\alpha)$ back to get

$$
G(\alpha)= \begin{cases}F(\alpha, b), & \alpha \geq 0 \\ -F(-\alpha, b)+2 F(0, b), & \alpha<0 .\end{cases}
$$

Therefore, we define $F(-\alpha, b)=2 F(0, b)-F(\alpha, b)$ for all $\alpha>0, b \in \mathbb{R}^{m}$. It is a standard construction in elementary calculus that the odd extension of a $C^{1}$ function (passing through the origin) is $C^{1}$. Therefore, it is easy to show that the function $F$, thus extended to all of $\mathbb{R} \times \mathbb{R}^{m}$, is $C^{1}$. Now we apply the implicit function theorem to show that $F(\alpha, b)=0$ has a unique solution for each $b$ sufficiently close to $b^{*}$. Let $b^{*} \in \operatorname{col}(A)$ be given. It follows that $F\left(0, b^{*}\right)=0$ and $\frac{\partial F}{\partial \alpha}\left(0, b^{*}\right)=-\left\|x_{0, b^{*}}\right\|^{2}<0$. (Note that we assume $b^{*} \notin N\left(A^{T}\right)$, and hence $x_{0, b^{*}} \neq 0$.) Therefore, the implicit function theorem applies. Then there exist $\delta_{1}, \delta_{2}>0$ and a $C^{1}$ function $r: B_{\delta_{2}}\left(b^{*}\right) \rightarrow\left(-\delta_{1}, \delta_{1}\right)$ such that for each $b \in B_{\delta_{2}}\left(b^{*}\right), \alpha=r(b)$ is the unique solution of $F(\alpha, b)=0$ in $\left(-\delta_{1}, \delta_{1}\right)$. 
We can also show that the solution $\alpha=r(b)$ of $F(\alpha, b)=0$ must satisfy $\alpha \geq 0$. For each $b \in B_{\delta_{2}}\left(b^{*}\right)$, we can apply the mean value theorem to find $t \in(0,1)$ such that

$$
F(\alpha, b)=F(0, b)+\frac{\partial F}{\partial \alpha}(t \alpha, b) \alpha
$$

If we take $\alpha=r(b)$, we obtain $F(0, b)+\frac{\partial F}{\partial \alpha}(t \alpha, b) \alpha=0$, which implies that $\frac{\partial F}{\partial \alpha}(t \alpha, b) \alpha=-F(0, b) \leq 0$. We just need to prove that $\frac{\partial F}{\partial \alpha}(t r(b), b)<0$ for all $b \in B_{\delta_{2}}\left(b^{*}\right)$. From the continuity of $\frac{\partial F}{\partial \alpha}$ and $r$, by reducing $\delta_{1}$ and $\delta_{2}$ if necessary, it follows that $\frac{\partial F}{\partial \alpha}(t \alpha, b)<0$, and hence that $\alpha=r(b) \geq 0$.

For $b \in \operatorname{col}(A), \alpha=r(b)=0$ and for $b \notin \operatorname{col}(A),\left\|A x_{\alpha, b}-b\right\|>0$ and hence $\left\|A x_{\alpha, b}-b\right\|^{2}-\alpha\left\|x_{\alpha, b}\right\|^{2}=0$ implies that $\alpha \neq 0$. Thus $\alpha=r(b)>0$ for $b \notin \operatorname{col}(A)$.

We have seen that $x_{\alpha, b}$ with $\alpha=r(b)$, is a stationary point of $J(\cdot ; b)$. We can show that it is a local minimizer of $J(\cdot ; b)$ by proving that $\nabla_{x x}^{2} J\left(x_{\alpha, b}, b\right)$ is positive definite. We have

$$
\begin{aligned}
\nabla_{x x}^{2} J(x, b) & =\nabla_{x x}^{2}\left(\frac{1}{2}\|A x-b\|^{2}\|x\|^{2}\right)=\nabla_{x}\left(\|x\|^{2}\left(A^{T} A x-A^{T} b\right)+\|A x-b\|^{2} x\right) \\
& =\nabla_{x}\left(\|x\|^{2}\right)\left(A^{T} A x-A^{T} b\right)+\|x\|^{2} \nabla_{x}\left(A^{T} A x-A^{T} b\right) \\
& +\nabla_{x}\left(\|A x-b\|^{2}\right) x+\|A x-b\|^{2} \nabla_{x}(x) \\
& =\|x\|^{2}\left(A^{T} A\right)+2\left(\left(A^{T} A x-A^{T} b\right) x^{T}+2 x\left(A^{T} A x-A^{T} b\right)^{T}\right)+\|A x-b\|^{2} I,
\end{aligned}
$$


and for $x=x_{\alpha, b}$, since $A^{T} A x_{\alpha, b}-A^{T} b=-\alpha x_{\alpha, b}$, then it follows that

$$
\begin{aligned}
\nabla_{x x}^{2} J\left(x_{\alpha, b}, b\right) & =\left\|x_{\alpha, b}\right\|^{2}\left(A^{T} A\right)-4 \alpha x_{\alpha, b} x_{\alpha, b}^{T}+\alpha\left\|x_{\alpha, b}\right\|^{2} I \\
& =\left\|x_{\alpha, b}\right\|^{2} N_{\alpha}-4 \alpha x_{\alpha, b} x_{\alpha, b}^{T} .
\end{aligned}
$$

We will now show that for $\alpha=r(b), J\left(x_{\alpha, b}, b\right)$ is positive definite by showing that $\left\langle v, \nabla_{x x}^{2} J\left(x_{\alpha, b}, b\right) v\right\rangle>0$ for every $v \in \mathbb{R}^{n}, v \neq 0$. We first show that every vector $v \in \mathbb{R}^{n}=U \oplus U^{\perp}$ (where $U=\operatorname{span}\left\{x_{\alpha, b}\right\}^{\perp}$ ) can be represented uniquely as $v=$ $u+\beta x_{\alpha, b}^{\prime}$, where $u \in U$ and $\beta \in \mathbb{R}$. We know that $x_{\alpha, b}^{\prime} \in \mathbb{R}^{n}$ can be written as $x_{\alpha, b}^{\prime}=\hat{u}+\gamma x_{\alpha, b}$ for $\hat{u} \in U, \gamma \in \mathbb{R}$ and $\gamma \neq 0$ (because if $\gamma=0$, then that implies $x_{\alpha, b}^{\prime} \in \operatorname{span}\left\{x_{\alpha, b}\right\}^{\perp}$ and $\left\langle x_{\alpha, b}, x_{\alpha, b}^{\prime}\right\rangle=0$, which is a contradiction for $\left.b \notin N\left(A^{T}\right)\right)$. It follows that $x_{\alpha, b}=\gamma^{-1} x_{\alpha, b}^{\prime}-\gamma^{-1} \hat{u}$ and for $v \in \mathbb{R}^{n}$ we have

$$
v=\bar{u}+\lambda x_{\alpha, b}=\bar{u}+\lambda\left(\gamma^{-1} x_{\alpha, b}^{\prime}-\gamma^{-1} \hat{u}\right)=\left(\bar{u}-\lambda \gamma^{-1} \hat{u}\right)+\lambda \gamma^{-1} x_{\alpha, b}^{\prime} .
$$

Thus $v=u+\beta x_{\alpha, b}^{\prime}$, where $u=\bar{u}-\lambda \gamma^{-1} \hat{u} \in U$ and $\beta=\lambda \gamma^{-1} \in \mathbb{R}$. Now suppose there exist $u_{1} \in U$ and $\beta_{1} \in \mathbb{R}$ such that $v=u+\beta x_{\alpha, b}^{\prime}=u_{1}+\beta_{1} x_{\alpha, b}^{\prime}$. Then we have $u-u_{1}=\beta_{1} x_{\alpha, b}^{\prime}-\beta x_{\alpha, b}^{\prime}$, where $u-u_{1} \in U$ and $\left(\beta_{1}-\beta\right) x_{\alpha, b}^{\prime} \in \mathbb{R}^{n}$. Therefore $\left(\beta_{1}-\beta\right) x_{\alpha, b}^{\prime}=u-u_{1} \in U$, that is, $\left(\beta_{1}-\beta\right)\left\langle x_{\alpha, b}, x_{\alpha, b}^{\prime}\right\rangle=0$ and therefore $\beta_{1}-\beta=0$ since $\left\langle x_{\alpha, b}, x_{\alpha, b}^{\prime}\right\rangle \neq 0$. Also it follows that $u-u_{1}=0$ and thus every $v \in \mathbb{R}^{n}$ has a unique representation. 
Now for all $v \in \mathbb{R}^{n}$, if $v=u+\beta x_{\alpha, b}^{\prime}$ and $v \neq 0$, then we have

$$
\begin{aligned}
\left\langle v, \nabla_{x x}^{2} J\left(x_{\alpha, b}, b\right) v\right\rangle & =\left\langle v,\left(\left\|x_{\alpha, b}\right\|^{2} N_{\alpha}-4 \alpha x_{\alpha, b} x_{\alpha, b}^{T}\right) v\right\rangle \\
& =\left\|x_{\alpha, b}\right\|^{2}\left\langle v, N_{\alpha} v\right\rangle-4 \alpha\left|\left\langle v, x_{\alpha, b}\right\rangle\right|^{2} \\
& =\left\|x_{\alpha, b}\right\|^{2}\left\langle\left(u+\beta x_{\alpha, b}^{\prime}\right), N_{\alpha}\left(u+\beta x_{\alpha, b}^{\prime}\right)\right\rangle-4 \alpha\left|\left\langle\left(u+\beta x_{\alpha, b}^{\prime}\right), x_{\alpha, b}\right\rangle\right|^{2} \\
& =\left\|x_{\alpha, b}\right\|^{2}\left(\left\langle u, N_{\alpha} u\right\rangle+2 \beta\left\langle x_{\alpha, b}^{\prime}, N_{\alpha} u\right\rangle+\beta^{2}\left\langle x_{\alpha, b}^{\prime}, N_{\alpha} x_{\alpha, b}^{\prime}\right\rangle\right)-4 \alpha\left|\left\langle\left(u+\beta x_{\alpha, b}^{\prime}\right), x_{\alpha, b}\right\rangle\right|^{2} \\
& =\left\|x_{\alpha, b}\right\|^{2}\left(\left\langle u, N_{\alpha} u\right\rangle-2 \beta\left\langle x_{\alpha, b}, u\right\rangle+\beta^{2}\left\langle x_{\alpha, b}^{\prime}, N_{\alpha} x_{\alpha, b}^{\prime}\right\rangle\right)-4 \alpha\left|\left\langle u, x_{\alpha, b}\right\rangle+\beta\left\langle x_{\alpha, b}^{\prime}, x_{\alpha, b}\right\rangle\right|^{2} \\
& =\left\|x_{\alpha, b}\right\|^{2}\left\langle u, N_{\alpha} u\right\rangle-\beta^{2}\left\|x_{\alpha, b}\right\|^{2}\left\langle x_{\alpha, b}, x_{\alpha, b}^{\prime}\right\rangle-4 \alpha \beta^{2}\left|\left\langle x_{\alpha, b}, x_{\alpha, b}^{\prime}\right\rangle\right|^{2}\left(\operatorname{since}\left\langle u, x_{\alpha, b}\right\rangle=0\right) \\
& =\left\|x_{\alpha, b}\right\|^{2}\left\langle u, N_{\alpha} u\right\rangle-\beta^{2}\left\langle x_{\alpha, b}, x_{\alpha, b}^{\prime}\right\rangle\left(\left\|x_{\alpha, b}\right\|^{2}+4 \alpha\left\langle x_{\alpha, b}, x_{\alpha, b}^{\prime}\right\rangle\right) \\
& =\left\|x_{\alpha, b}\right\|^{2}\left\langle u, N_{\alpha} u\right\rangle+\beta^{2}\left\langle x_{\alpha, b}, N_{\alpha} x_{\alpha, b}\right\rangle\left(\left\|x_{\alpha, b}\right\|^{2}-4 \alpha\left\langle x_{\alpha, b}, N_{\alpha}^{-1} x_{\alpha, b}\right\rangle\right) .
\end{aligned}
$$

Recall that $\frac{\partial F}{\partial \alpha}(\alpha, b)=4 \alpha\left\langle x_{\alpha, b}, N_{\alpha} x_{\alpha, b}\right\rangle-\left\|x_{\alpha, b}\right\|^{2}<0$ for $\alpha=r(b)$. Also if $v \neq 0$, then $u \neq 0$ or $\beta \neq 0$ (or both). If $u \neq 0$, then $\left\langle u, N_{\alpha} u\right\rangle>0$ and if $\beta \neq 0$, then

$$
\beta^{2}\left\langle x_{\alpha, b}, N_{\alpha} x_{\alpha, b}\right\rangle\left(\left\|x_{\alpha, b}\right\|^{2}-4 \alpha\left\langle x_{\alpha, b}, N_{\alpha}^{-1} x_{\alpha, b}\right\rangle\right)>0
$$

Therefore,

$$
\left\langle v, \nabla_{x x}^{2} J\left(x_{\alpha, b}, b\right) v\right\rangle=\left\|x_{\alpha, b}\right\|^{2}\left\langle u, N_{\alpha} u\right\rangle+\beta^{2}\left\langle x_{\alpha, b}, N_{\alpha} x_{\alpha, b}\right\rangle\left(\left\|x_{\alpha, b}\right\|^{2}-4 \alpha\left\langle x_{\alpha, b}, N_{\alpha}^{-1} x_{\alpha, b}\right\rangle\right)>0 .
$$

We now see that there exists $\delta_{2}>0$ such that for all $b \in B_{\delta_{2}}\left(b^{*}\right) \backslash \operatorname{col}(A), J(\cdot ; b)$ has a local minimizer $x_{\alpha, b}$, where $\alpha=r(b)$ satisfies $\alpha=\frac{\left\|A x_{\alpha, b}-b\right\|^{2}}{\left\|x_{\alpha, b}\right\|^{2}}$. We can also say that this $\alpha$ is a local minimizer of $\varphi(\cdot ; b)$. 


\subsection{Rate of convergence}

The standard theory of Tikhonov regularization for an infinite-dimensional problem shows that if $\alpha=r(b) \rightarrow 0$ as $b \rightarrow b^{*}$ and

- $\frac{\left\|b-b^{*}\right\|^{2}}{\alpha} \rightarrow 0$, then $x_{\alpha, b} \rightarrow x_{0, b^{*}}$ strongly;

- $\frac{\left\|b-b^{*}\right\|^{2}}{\alpha} \leq C$, then $x_{\alpha, b} \rightarrow x_{0, b^{*}}$ weakly;

$-\frac{\left\|b-b^{*}\right\|^{2}}{\alpha} \rightarrow \infty, x_{\alpha, b} \nrightarrow x_{0, b^{*}}\left(\left\|x_{\alpha, b}\right\| \rightarrow \infty\right)$.

In a finite-dimensional problem, $\alpha=r(b) \rightarrow 0$ as $b \rightarrow b^{*}$ is sufficient to imply that $x_{\alpha, b} \rightarrow x_{0, b^{*}}$. This can be proved as follows

$$
\begin{aligned}
\left\|x_{\alpha, b}-x_{0, b^{*}}\right\| & =\left\|x_{\alpha, b}-x_{\alpha, b^{*}}+x_{\alpha, b^{*}}-x_{0, b^{*}}\right\| \leq\left\|N_{\alpha}^{-1} T^{*}\left(b-b^{*}\right)\right\|+\left\|x_{\alpha, b^{*}}-x_{0, b^{*}}\right\| \\
& \leq\left\|N_{\alpha}^{-1} T^{*}\right\|\left\|b-b^{*}\right\|+\left\|x_{\alpha, b^{*}}-x_{0, b^{*}}\right\| \\
& \leq\left\|T^{\dagger}\right\|\left\|b-b^{*}\right\|+\left\|x_{\alpha, b^{*}}-x_{0, b^{*}}\right\| \rightarrow 0, \text { as } b \rightarrow b^{*} .
\end{aligned}
$$

Nevertheless, since most interesting finite-dimensional problems arise from discretizing infinite-dimensional problems, we would like to investigate the behavior of $\frac{\left\|b-b^{*}\right\|^{2}}{\alpha}$ as $b \rightarrow b^{*}$. 
Let $b \in \mathbb{R}^{m}$. We define a new function

$$
f(\alpha, b)=\|\hat{b}\|^{2}-F(\alpha, b)= \begin{cases}\alpha\left\|x_{\alpha, b}\right\|^{2}-\left\|A x_{\alpha, b}-\bar{b}\right\|^{2}, & \alpha>0 \\ 0, & \alpha=0 .\end{cases}
$$

Then $F(\alpha, b)=0$ is equivalent to $f(\alpha, b)=\|\hat{b}\|^{2}$. Thus $\alpha=r(b)$ satisfies

$$
\alpha=\frac{\|\hat{b}\|^{2}}{\left\|x_{\alpha, b}\right\|^{2}-\alpha^{-1}\left\|A x_{\alpha, b}-\bar{b}\right\|^{2}} .
$$

We can show that $\lim _{(\alpha, b) \rightarrow\left(0, b^{*}\right)}\left(\left\|x_{\alpha, b}\right\|^{2}-\alpha^{-1}\left\|A x_{\alpha, b}-\bar{b}\right\|^{2}\right)=\left\|x_{0, b^{*}}\right\|^{2}$.

That is true because

$$
\begin{aligned}
\lim _{(\alpha, b) \rightarrow\left(0, b^{*}\right)}\left(\left\|x_{\alpha, b}\right\|^{2}-\alpha^{-1}\left\|A x_{\alpha, b}-\bar{b}\right\|^{2}\right) & =\lim _{(\alpha, b) \rightarrow\left(0, b^{*}\right)}\left(\sum_{i=1}^{r} \frac{\alpha-\sigma_{i}^{2}}{\left(\sigma_{i}^{2}+\alpha\right)^{2}}\left|\left\langle b, \psi_{i}\right\rangle\right|^{2}+\sum_{i=r+1}^{m}\left|\left\langle b, \psi_{i}\right\rangle\right|^{2}\right) \\
& \left.=\lim _{(\alpha, b) \rightarrow\left(0, b^{*}\right)} \sum_{i=1}^{r} \frac{\alpha-\sigma_{i}^{2}}{\left(\sigma_{i}^{2}+\alpha\right)^{2}}\left|\left\langle b, \psi_{i}\right\rangle\right|^{2}+\lim _{b \rightarrow b^{*}} \sum_{i=r+1}^{m}\left|\left\langle b, \psi_{i}\right\rangle\right|^{2}\right) \\
& =-\sum_{i=1}^{r} \frac{\left|\left\langle b^{*}, \psi_{i}\right\rangle\right|^{2}}{\sigma_{i}^{2}}+\sum_{i=r+1}^{m}\left|\left\langle b^{*}, \psi_{i}\right\rangle\right|^{2}=\left\|x_{0, b^{*}}\right\|^{2} .
\end{aligned}
$$

Notice that $\left\langle b^{*}, \psi_{i}\right\rangle=0$ for all $i=r+1, \ldots, n$ because $b^{*} \in \operatorname{col}(A)=$ $\operatorname{span}\left\{\psi_{r+1}, \ldots, \psi_{n}\right\}^{\perp}$. Therefore,

$$
\frac{\|\hat{b}\|^{2}}{\alpha}=\left\|x_{\alpha, b}\right\|^{2}-\alpha^{-1}\left\|A x_{\alpha, b}-\bar{b}\right\|^{2} \rightarrow\left\|x_{0, b^{*}}\right\|^{2} \text { as } b \rightarrow b^{*}
$$




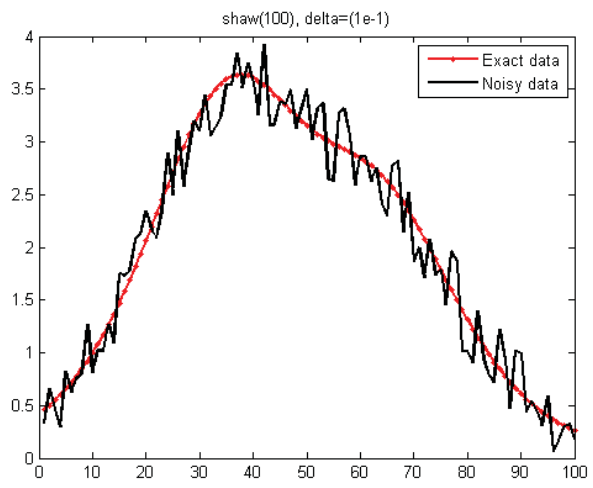

Figure 3.1: The exact data and the noisy data for the test problem "shaw" $(\mathrm{n}=100)$, with relative error $10^{-1}$ in the noisy data.

where $\alpha=r(b)$. We see that $\alpha$ goes to zero at the same rate as $\|\hat{b}\|^{2}$. This analysis is meaningful if $\hat{b} \neq 0$, which is not possible if $\operatorname{col}(A)$ is all of $\mathbb{R}^{m}$. If $\operatorname{col}(A)=\mathbb{R}^{m}$, then multiplicative regularization does not work, that is, it produces $\alpha=r(b)=0$.

\subsubsection{Numerical examples}

For our numerical experiments, we use eight test problems from Hansen's Regularization Tools package [22]. See Section 1.6 for a description of these problems. For each experiment, we generate noisy vectors $b$ for several different relative noise levels $\delta=\frac{\left\|b-b^{*}\right\|}{\left\|b^{*}\right\|}$ (see Figure 3.1 for a typical example). The components of the noise vector are uniformly distributed random numbers selected from an interval centered at zero.

Our numerical tests verify that $\alpha \rightarrow 0$ as $\delta \rightarrow 0$, where $\alpha$ is the regularization parameter selected by multiplicative regularization. If we fit $\alpha$ to a formula of the 
Table 3.1

The rate of convergence for multiplicative regularization

\begin{tabular}{|c|c|}
\hline Multiplicative Regularization & \\
\hline Test problem & $\mathrm{p}$ \\
\hline deriv2 & - \\
\hline phillips & - \\
\hline heat & 2.288 \\
\hline i_laplace & 1.9565 \\
\hline foxgood & 2.0097 \\
\hline shaw & 2.0134 \\
\hline baart & 2.0228 \\
\hline wing & 2.0692 \\
\hline
\end{tabular}

form $\alpha=C \delta^{p}$ (where $C$ and $p$ are constant), then we find the values of $p$ shown in Table 3.1 for the test problems.

Multiplicative regularization fails on "deriv2" and "phillips", which will be discussed in Section 3.5.1. The test problem "heat" is a special case and the convergence is slightly faster than predicted. That is probably because most of the components of $b$ are in the $\operatorname{col}(A)(A$ is $100 \times 100$ and $\operatorname{rank}(A)=97)$. For the other problems, the results are as expected.

\subsection{Comparison with the L-curve method}

To see the performance of multiplicative regularization, we compare the computed solutions with results obtained by the L-curve method for different noise levels. We 


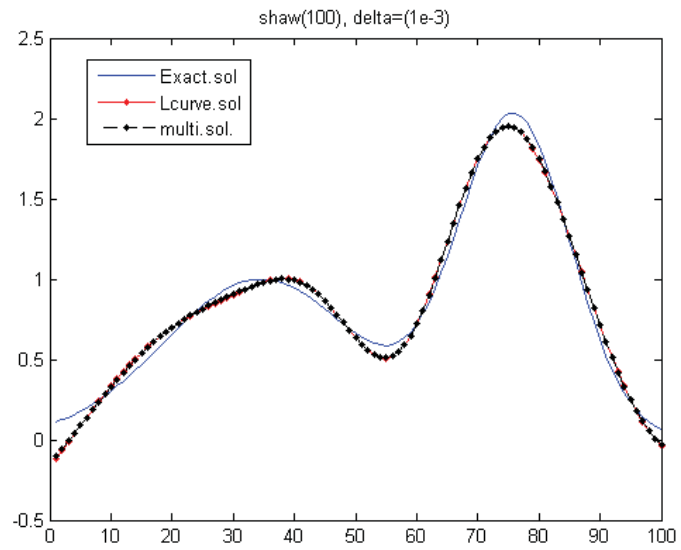

Figure 3.2: Tikhonov regularization: The exact solution for the "shaw" problem, together with the regularized solutions produced by the L-curve method and multiplicative regularization. In this example, the results of the two methods are essentially the same.

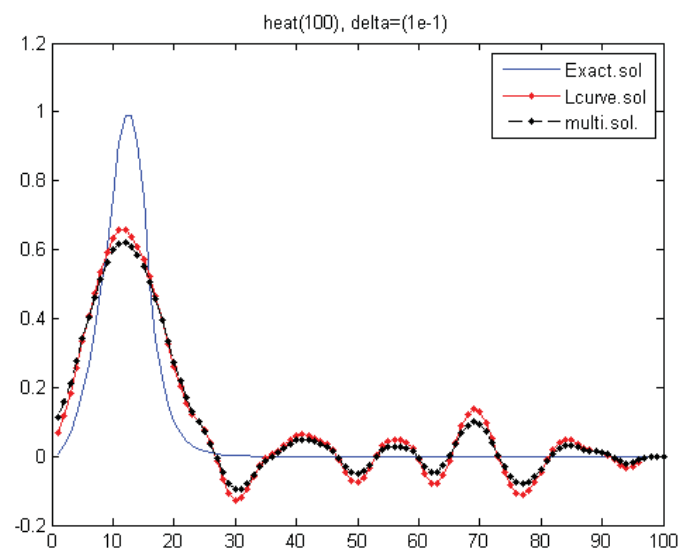

Figure 3.3: Tikhonov regularization: The exact solution for the "heat" problem, together with the regularized solutions produced by the L-curve method and multiplicative regularization. In this example, the two methods produce similar estimates, but the L-curve estimate is slightly better.

compare the solutions produced by both methods on the basis of the error $\frac{\left\|x-x^{*}\right\|}{\left\|x^{*}\right\|}$.

We classify the results of each experiment into one of three categories: we use (MR) if 


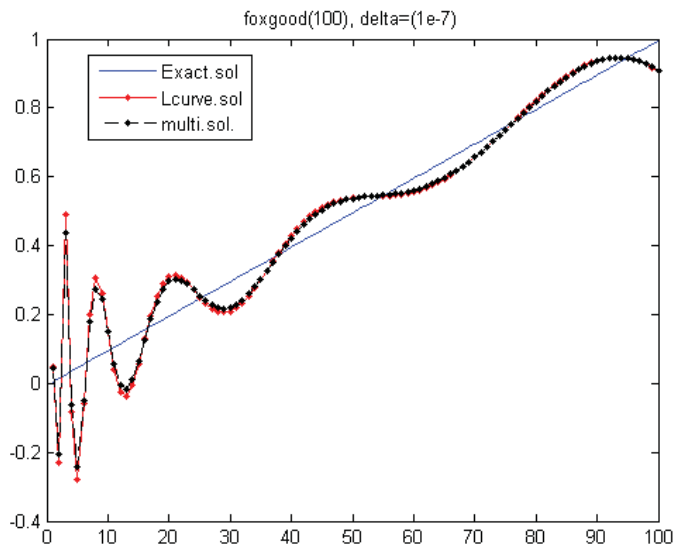

Figure 3.4: The exact solution for the "foxgood" problem, together with the regularized solutions produced by the L-curve method and multiplicative regularization. In this example, the two methods produce similar estimates, but multiplicative regularization estimate is slightly better.

multiplicative regularization produces a better solution, (LC) if the L-curve method performs better and (-) if the results are essentially the same. One solution is classified as the better solution if the error of that solution is at least $10 \%$ smaller than the error of the other one. We tested the method on eight problems with seven noise levels for each, a total of 56 cases. On four problems (28 cases), the performance of the two methods is essentially identical (see Figure 3.2 for a typical example). On two problems (14 cases), the performance is similar, but sometimes the L-curve method is slightly better (see Figure 3.2) and sometimes multiplicative regularization is somewhat better (see Figure 3.3). In these cases, L-curve is usually the better method. On two problems (14 cases), multiplicative regularization fails for the reason discussed earlier. The L-curve method also failed in three of these 14 cases. 
Table 3.2

Comparison of multiplicative regularization with the L-curve method

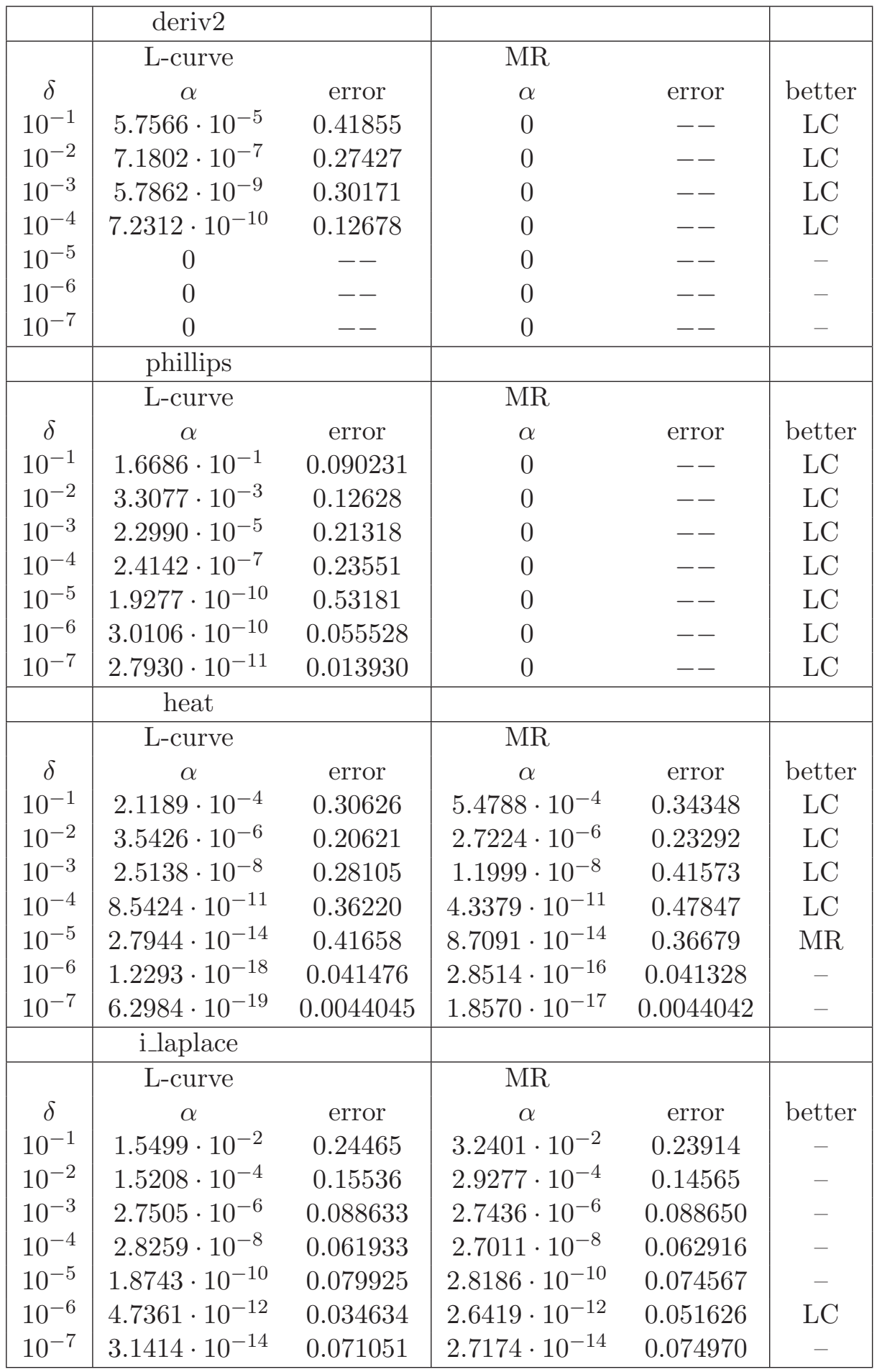


Table 3.3

Comparison of multiplicative regularization with the L-curve method

\begin{tabular}{|c|c|c|c|c|c|}
\hline & \multicolumn{2}{|l|}{ foxgood } & \multirow{2}{*}{\multicolumn{2}{|c|}{ MR }} & \multirow[b]{3}{*}{ better } \\
\hline & L-curve & & & & \\
\hline$\delta$ & $\alpha$ & error & $\alpha$ & error & \\
\hline $10^{-1}$ & $2.0157 \cdot 10^{-3}$ & 0.11777 & $6.2084 \cdot 10^{-3}$ & 0.16537 & $\mathrm{LC}$ \\
\hline $10^{-2}$ & $5.0232 \cdot 10^{-5}$ & 0.039497 & $5.8650 \cdot 10^{-5}$ & 0.038102 & - \\
\hline $10^{-3}$ & $6.9384 \cdot 10^{-7}$ & 0.020652 & $5.6384 \cdot 10^{-7}$ & 0.022084 & - \\
\hline $10^{-4}$ & $8.9824 \cdot 10^{-9}$ & 0.059639 & $5.7022 \cdot 10^{-9}$ & 0.072235 & $\mathrm{LC}$ \\
\hline $10^{-5}$ & $1.6246 \cdot 10^{-10}$ & 0.029053 & $5.5865 \cdot 10^{-11}$ & 0.057751 & $\mathrm{LC}$ \\
\hline $10^{-6}$ & $1.0776 \cdot 10^{-12}$ & 0.017196 & $4.8795 \cdot 10^{-13}$ & 0.025274 & $\mathrm{LC}$ \\
\hline \multirow{2}{*}{$10^{-7}$} & $3.6618 \cdot 10^{-15}$ & 0.10967 & $5.3269 \cdot 10^{-15}$ & 0.095239 & MR \\
\hline & \multicolumn{2}{|l|}{ shaw } & & & \\
\hline & \multicolumn{2}{|l|}{ L-curve } & \multicolumn{2}{|l|}{ MR } & \\
\hline$\delta$ & $\alpha$ & error & $\alpha$ & error & better \\
\hline $10^{-1}$ & $2.4366 \cdot 10^{-2}$ & 0.18347 & $5.9525 \cdot 10^{-2}$ & 0.18632 & - \\
\hline $10^{-2}$ & $3.8168 \cdot 10^{-4}$ & 0.086376 & $5.1872 \cdot 10^{-4}$ & 0.080728 & - \\
\hline $10^{-3}$ & $4.8446 \cdot 10^{-6}$ & 0.084472 & $5.0056 \cdot 10^{-6}$ & 0.083831 & - \\
\hline $10^{-4}$ & $4.4892 \cdot 10^{-8}$ & 0.043844 & $4.9867 \cdot 10^{-8}$ & 0.044030 & - \\
\hline $10^{-5}$ & $8.1194 \cdot 10^{-10}$ & 0.049761 & $4.9749 \cdot 10^{-10}$ & 0.053016 & - \\
\hline $10^{-6}$ & $5.3854 \cdot 10^{-12}$ & 0.029093 & $4.9204 \cdot 10^{-12}$ & 0.029143 & - \\
\hline \multirow[t]{2}{*}{$10^{-7}$} & $1.8301 \cdot 10^{-14}$ & 0.066169 & $4.4538 \cdot 10^{-14}$ & 0.061199 & - \\
\hline & \multicolumn{2}{|l|}{ baart } & & & \\
\hline & \multicolumn{2}{|l|}{ L-curve } & \multicolumn{2}{|l|}{ MR } & \\
\hline$\delta$ & $\alpha$ & error & $\alpha$ & error & better \\
\hline $10^{-1}$ & $1.8203 \cdot 10^{-2}$ & 0.38809 & $7.4751 \cdot 10^{-2}$ & 0.37298 & - \\
\hline $10^{-2}$ & $2.0462 \cdot 10^{-4}$ & 0.15442 & $5.5709 \cdot 10^{-4}$ & 0.16481 & - \\
\hline $10^{-3}$ & $2.3146 \cdot 10^{-6}$ & 0.13694 & $5.1749 \cdot 10^{-6}$ & 0.12438 & - \\
\hline $10^{-4}$ & $5.2228 \cdot 10^{-8}$ & 0.059095 & $5.0171 \cdot 10^{-8}$ & 0.058916 & - \\
\hline $10^{-5}$ & $6.7614 \cdot 10^{-10}$ & 0.061845 & $5.2440 \cdot 10^{-10}$ & 0.064648 & - \\
\hline $10^{-6}$ & $6.2654 \cdot 10^{-12}$ & 0.053637 & $5.2037 \cdot 10^{-12}$ & 0.054689 & - \\
\hline \multirow[t]{2}{*}{$10^{-7}$} & $2.9745 \cdot 10^{-14}$ & 0.032913 & $4.8572 \cdot 10^{-14}$ & 0.034044 & - \\
\hline & \multicolumn{2}{|l|}{ wing } & & & \\
\hline & \multicolumn{2}{|l|}{ L-curve } & \multicolumn{2}{|l|}{ MR } & \\
\hline$\delta$ & $\alpha$ & error & $\alpha$ & error & better \\
\hline $10^{-1}$ & $3.8860 \cdot 10^{-3}$ & 0.73238 & $2.4572 \cdot 10^{-3}$ & 0.70125 & - \\
\hline $10^{-2}$ & $3.1947 \cdot 10^{-6}$ & 0.59493 & $1.0035 \cdot 10^{-5}$ & 0.59475 & - \\
\hline $10^{-3}$ & $5.5346 \cdot 10^{-8}$ & 0.59242 & $9.8831 \cdot 10^{-8}$ & 0.59341 & - \\
\hline $10^{-4}$ & $1.9538 \cdot 10^{-9}$ & 0.56667 & $9.6922 \cdot 10^{-10}$ & 0.54962 & - \\
\hline $10^{-5}$ & $3.4017 \cdot 10^{-12}$ & 0.44772 & $7.4998 \cdot 10^{-12}$ & 0.44230 & - \\
\hline $10^{-6}$ & $3.1521 \cdot 10^{-14}$ & 0.35107 & $7.5627 \cdot 10^{-14}$ & 0.36499 & - \\
\hline $10^{-7}$ & $4.0807 \cdot 10^{-16}$ & 0.32416 & $6.8258 \cdot 10^{-16}$ & 0.32399 & - \\
\hline
\end{tabular}




\subsubsection{The failure of multiplicative regularization on "deriv2" and "phillips" test problems}

The analysis shows that multiplicative regularization chooses a regularization parameter of zero when the noisy data vector lies in the column space of the matrix.

The test problems "deriv2" and "phillips" are both obtained by discretization of first kind Fredholm integral equations. The infinite-dimensional problems are both mildly ill-posed, that is, their singular values decay slowly to zero. In both cases, discretization yields a square, nonsingular matrix $A$ and hence multiplicative regularization chooses $\alpha=0$ for every $b$ lies in the column space of $A$ (see Figures 3.7, 3.8, 3.9 and 3.10). Also for "deriv2", the L-curve method fails itself for smaller noise levels (see Figure 3.10). In these cases, the L-curve does not have a corner.

We expect multiplicative regularization to work well on "deriv2" and "phillips" if the problem are discretized so that $A \in \mathbb{R}^{m \times n}, m>n$. Then $b \notin \operatorname{col}(A)$ is likely to hold if $b$ contains random noise. We discretized these problems to obtain $A \in \mathbb{R}^{m \times n}, m=2 n$. This corresponds to collecting more data. As shown in Table 3.4, multiplicative regularization works well with these discretizations and produces better solutions than does the L-curve most of the time (see Figures 3.11, 3.12, 3.13 and 3.14). 
Table 3.4

Comparison of multiplicative regularization with the L-curve method

\begin{tabular}{|c|cc|cc|c|}
\hline & Re-discretized deriv2 & & & \\
\hline & L-curve & & multi-reg & \\
$\delta$ & $\alpha$ & error & $\alpha$ & error & better \\
$10^{-1}$ & $2.6408 \cdot 10^{-5}$ & 0.34582 & $9.3739 \cdot 10^{-5}$ & 0.39561 & LC \\
$10^{-2}$ & $3.0159 \cdot 10^{-7}$ & 0.24397 & $6.7902 \cdot 10^{-7}$ & 0.23069 & - \\
$10^{-3}$ & $4.3886 \cdot 10^{-9}$ & 0.21248 & $5.7205 \cdot 10^{-9}$ & 0.19077 & MR \\
$10^{-4}$ & $8.8420 \cdot 10^{-11}$ & 0.22389 & $3.3278 \cdot 10^{-11}$ & 0.35279 & MR \\
$10^{-5}$ & $2.6816 \cdot 10^{-11}$ & 0.056869 & $3.3157 \cdot 10^{-13}$ & 0.012736 & MR \\
$10^{-6}$ & 0 & -- & $2.8832 \cdot 10^{-15}$ & 0.014071 & MR \\
$10^{-7}$ & 0 & -- & $3.1382 \cdot 10^{-17}$ & 0.0014544 & MR \\
\hline & Re-discretized phillips & & & & \\
\hline & L-curve & & multi-reg & & \\
$\delta$ & $\alpha$ & error & $\alpha$ & error & better \\
$10^{-1}$ & $5.8377 \cdot 10^{-2}$ & 0.067864 & $2.6483 \cdot 10^{-1}$ & 0.037441 & MR \\
$10^{-2}$ & $1.6060 \cdot 10^{-3}$ & 0.099886 & $2.4835 \cdot 10^{-3}$ & 0.075813 & MR \\
$10^{-3}$ & $2.0743 \cdot 10^{-5}$ & 0.096715 & $2.3827 \cdot 10^{-5}$ & 0.089421 & - \\
$10^{-4}$ & $1.0972 \cdot 10^{-7}$ & 0.28569 & $1.9927 \cdot 10^{-7}$ & 0.20812 & MR \\
$10^{-5}$ & $2.7038 \cdot 10^{-9}$ & 0.23465 & $1.4817 \cdot 10^{-9}$ & 0.32184 & LC \\
$10^{-6}$ & $4.7798 \cdot 10^{-9}$ & 0.036692 & $6.7599 \cdot 10^{-11}$ & 0.32768 & MR \\
$10^{-7}$ & $5.5115 \cdot 10^{-9}$ & 0.029525 & $5.3987 \cdot 10^{-11}$ & 0.31202 & LC \\
\hline
\end{tabular}

\subsubsection{More details about the numerical examples}

In this section, we will look carefully at some problems for which the two methods perform essentially the same and deal with some other problems for which the two methods differ noticeably.

Figures 3.5 and 3.6 show four typical cases, in which both methods work well. We graph the curvature of the L-curve and the function $\varphi(\cdot ; b)$ in each figure. In each case, the curvature has a well-defined maximum and $\varphi$ has a well-defined minimum. The two values are similar. 

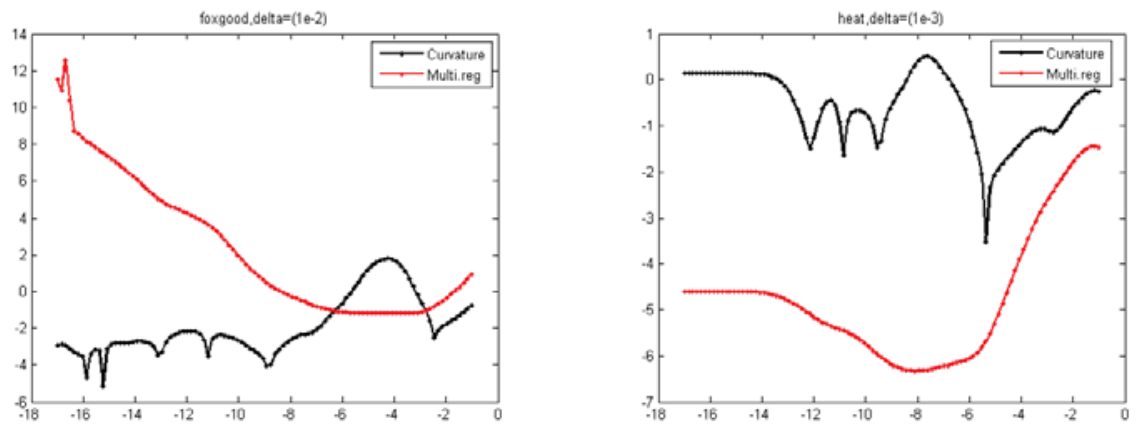

Figure 3.5: The test problem "foxgood", with the noise level $10^{-2}$ on the left and the test problem "heat", with the noise level $10^{-3}$ on the right. Both methods perform well.
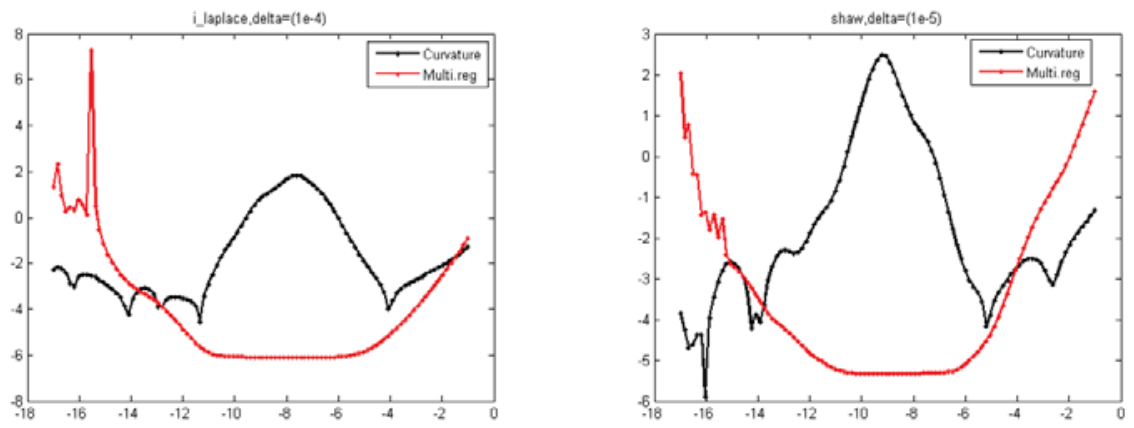

Figure 3.6: The test problem "ilaplace", with the noise level $10^{-4}$ on the left and the test problem "shaw", with the noise level $10^{-5}$ on the right. Both methods perform well.

Next, we present some cases in which the two methods differ. Figures 3.7, 3.8 and 3.9 are examples for which the L-curve method succeeds but the multiplicative regularization method fails because $b \in \operatorname{col}(A)$. Notice that for these three cases, the curvature of the L-curve has a well-defined maximum. However, the smallest minimizer of $\varphi(\cdot ; b)$, which is the value of $\alpha$ chosen by multiplicative regularization, is $\alpha=0$ for all three problems. 

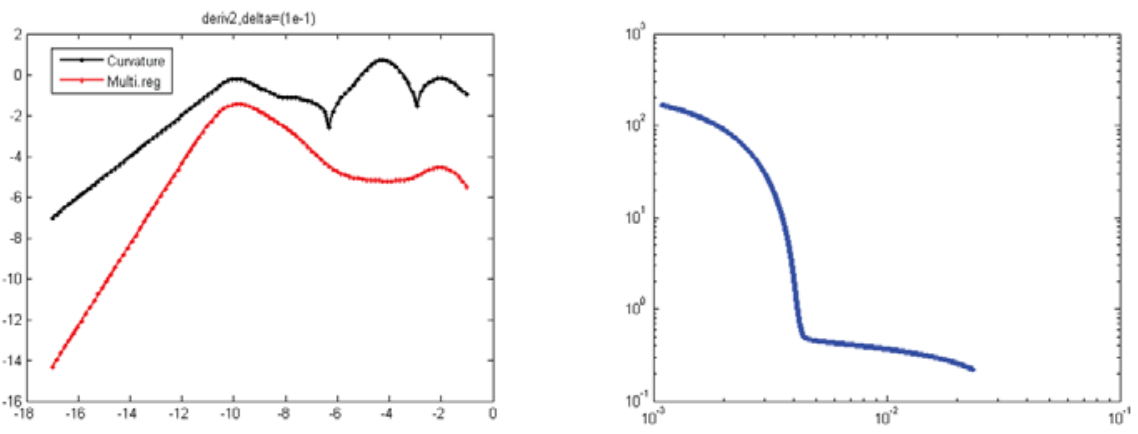

Figure 3.7: The test problem "deriv2" with relative error $\delta=10^{-1}$ in the noisy data. The curvature has a well-defined corner but multiplicative regularization fails. The graph on the right is the graph of the L-curve.
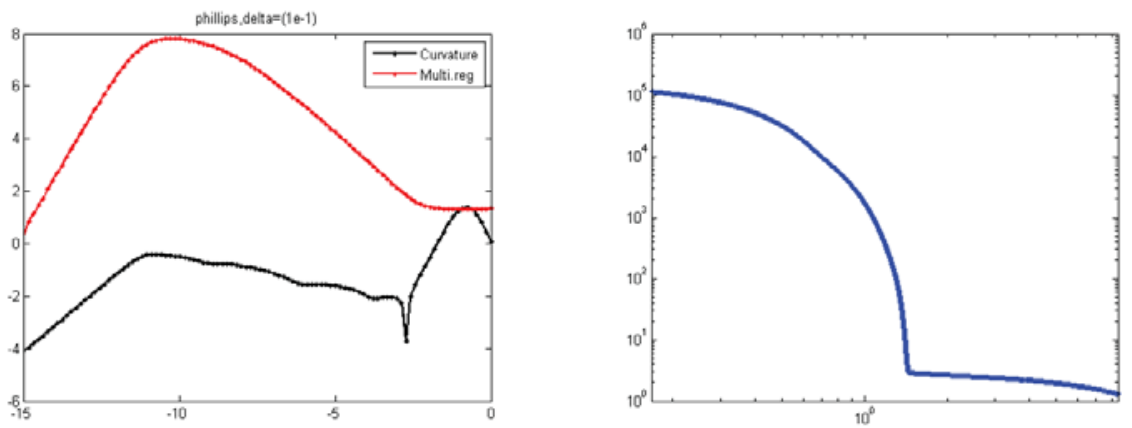

Figure 3.8: The test problem "phillips" with the noise level $\delta=10^{-1}$ in the noisy data. The curvature has a well-defined corner but multiplicative regularization fails. The graph on the right is the graph of the L-curve.
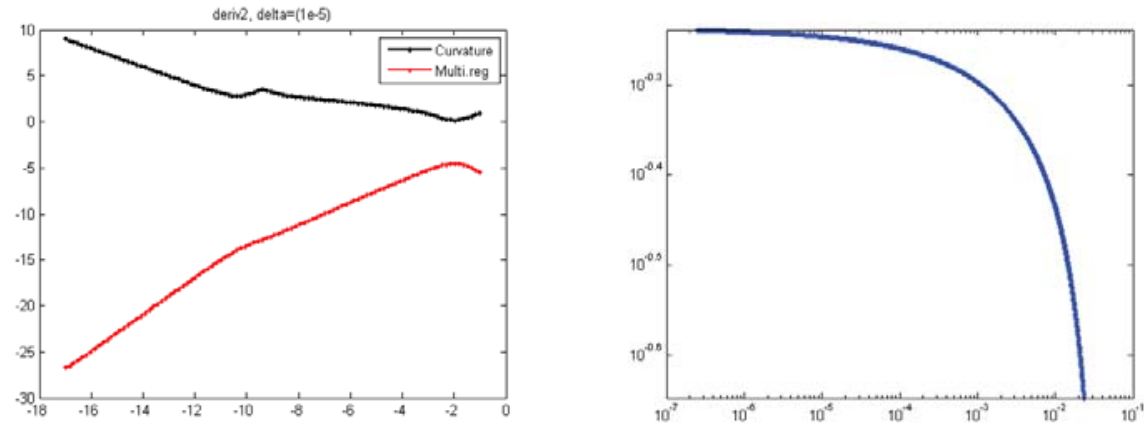

Figure 3.9: The test problem "deriv2" with relative error $\delta=10^{-5}$ in the noisy data. Both methods fail to identify nonzero regularization parameters. The graph on the right is the graph of the L-curve and it does not have a corner. 

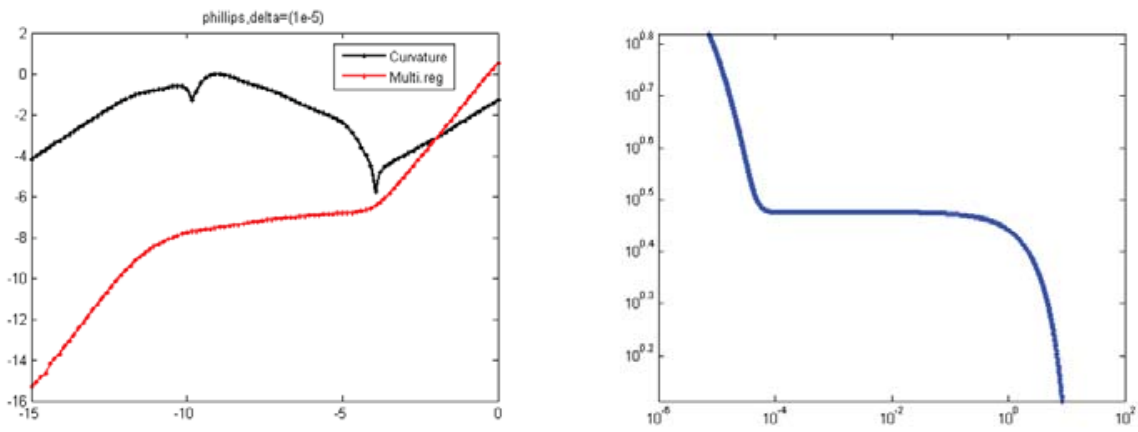

Figure 3.10: The test problem "phillips" with relative error $\delta=10^{-5}$ in the noisy data. The curvature has a well-defined corner but multiplicative regularization fails. The graph on the right is the graph of the L-curve.

Figure 3.10 is an example for which the L-curve method and the multiplicative regularization method both fail. Notice that the curvature of the L-curve does not have a well-defined maximum and the smallest minimizer of $\varphi(\cdot ; b)$ is $\alpha=0$.

Figures 3.11 and 3.12 are examples of re-discretized test problems "deriv2" and "phillips" with relative error $\delta=10^{-1}$ in the noisy data. The L-curve method succeeds and the multiplicative regularization method does not fail anymore since $b \notin \operatorname{col}(A)$. Notice that for both cases, the curvature of the L-curve has a well-defined maximum and $\varphi(\cdot ; b)$ has a well-defined minimum.

Figure 3.13 is an example of (re-discretized) test problems "phillips" with relative error $\delta=10^{-5}$ in the noisy data. Notice that the curvature of the L-curve has a well-defined maximum and $\varphi(\cdot ; b)$ has a well-defined minimum. Since the smallest minimizer of $\varphi(\cdot ; b)$ is not clear, we graph the fixed-point equation $\beta=f_{b}(\alpha)$ (where $f_{b}(\alpha)=$ $\left.\frac{\left\|A x_{\alpha, b}-b\right\|^{2}}{\left\|x_{\alpha, b}\right\|^{2}}\right)$. It is clear that the fixed-point equation finds a nonzero regularization 

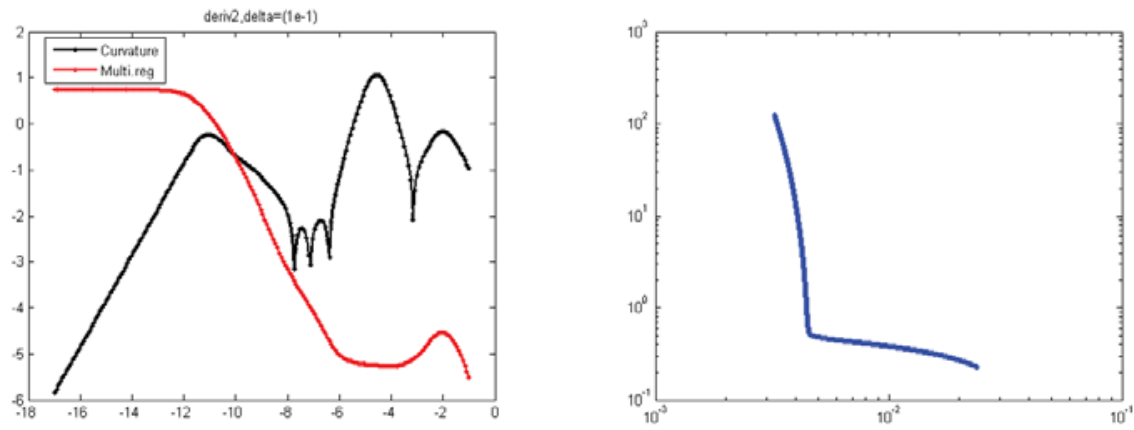

Figure 3.11: The re-descretized test problem "deriv2" with relative error $\delta=10^{-1}$ in the noisy data. Both methods identify nonzero regularization parameters. The graph on the right is the graph of the L-curve and it has a well-defined corner.
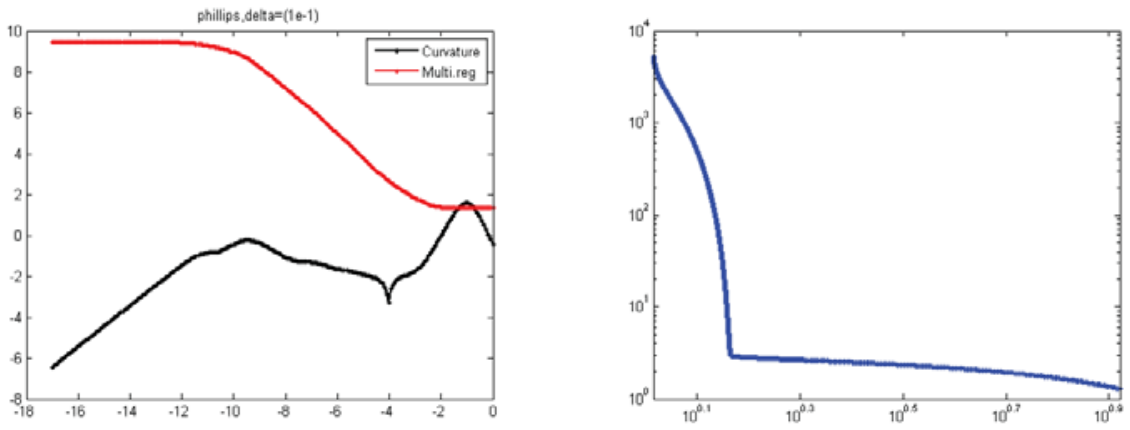

Figure 3.12: The re-discretized test problem "phillips" with relative error $\delta=10^{-1}$ in the noisy data. Both methods identify nonzero regularization parameters. The graph on the right is the graph of the L-curve and has a well-defined corner.

parameter.

Figures 3.14 is an example of (re-discretized) test problems "deriv2" with relative error $\delta=10^{-5}$ in the noisy data. The L-curve method fails since the curvature of the L-curve does not have a well-defined corner; however, $\varphi(\cdot ; b)$ has a well-defined minimum. Multiplicative regularization performs well since the fixed point equation finds a nonzero regularization parameter. 

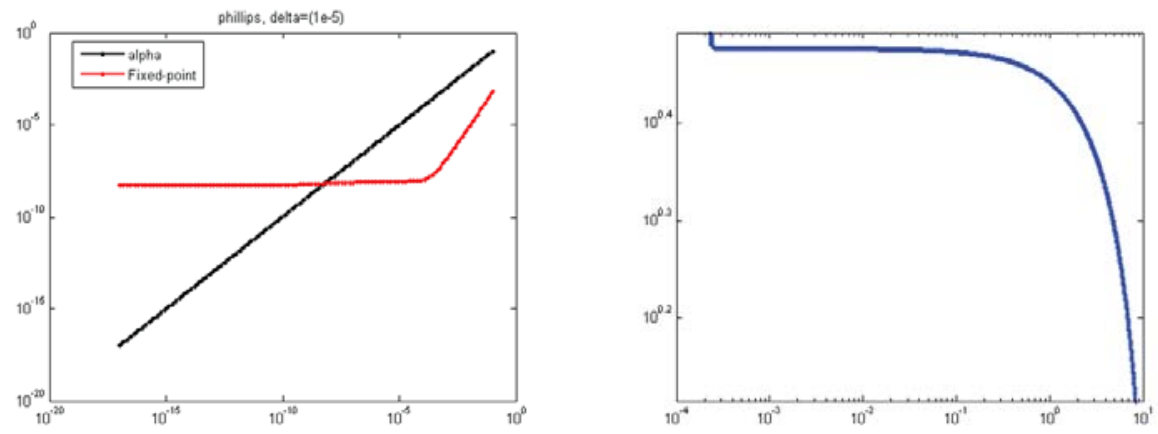

Figure 3.13: The re-discretized test problem "phillips" with relative error $\delta=10^{-5}$ in the noisy data. Both methods identify nonzero regularization parameters.
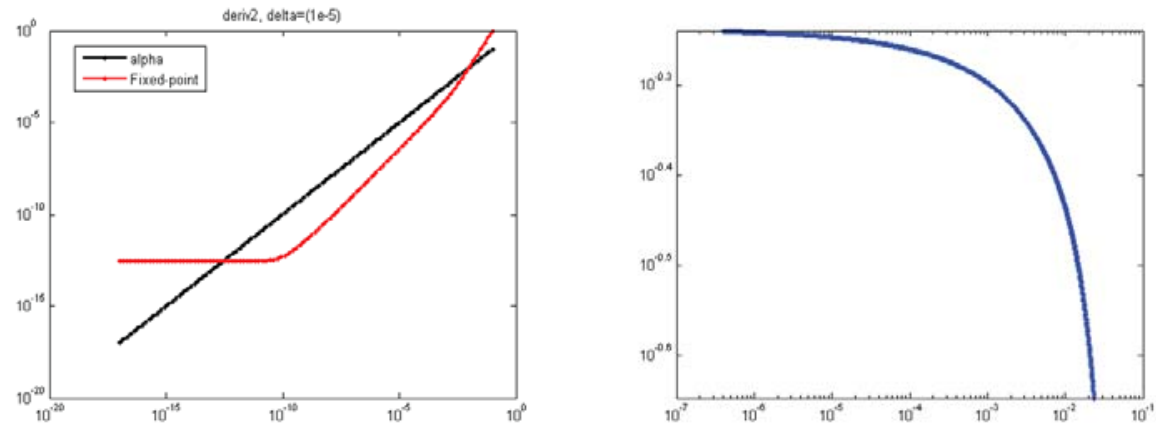

Figure 3.14: The re-discretized test problem "deriv2" with relative error $\delta=10^{-5}$ in the noisy data. The multiplicative regularization identifies a nonzero regularization parameter and the L-curve method fails to identify a corner.

\subsection{Conclusions}

Based on the numerical results, both method perform remarkably similarly provided $A \in \mathbb{R}^{m \times n}$ and $\operatorname{col}(A)$ is a proper subspace of $\mathbb{R}^{m}$. Multiplicative regularization is easy to analyze, while there are no analytic results for L-curve. For instance, we cannot prove that the L-curve always has a corner; indeed, we have just seen examples 
in which it does not. On the other hand, provided $b \notin \operatorname{col}(A)$ and $b$ is sufficiently close to $b^{*}$, we know that multiplicative regularization defines a positive regularization parameter $\alpha$. Moreover, we know that $\alpha \rightarrow 0$ at the same rate as $\|\hat{b}\|^{2}$ as $b \rightarrow b^{*}$.

We know that for an infinite-dimensional inverse problem, a purely a posteriori parameter choice method can not be convergent. However, if we prove that these results extend to infinite dimensions, namely, that

$$
y \rightarrow y^{*},\|\hat{y}\|_{Y} \geq \theta\left\|y-y^{*}\right\|_{Y} \Rightarrow \alpha(y) \rightarrow 0 \text { like }\|\hat{y}\|_{Y}^{2}
$$

then we will obtain $x_{\alpha, y} \rightarrow x^{*}$ weakly. We know that as $y_{k} \rightarrow y$ and even if $\left\{y_{k}\right\}$ is almost contained in $R(T)$, then multiplicative regularization probably fails. We need to ensure that each $y_{k}$ contains a significant component in $R(T)^{\perp}$. The condition $\|\hat{y}\|_{Y} \geq \theta\left\|y-y^{*}\right\|_{Y}$ is necessary in order to avoid the asymptotic convergence of $y$ to $y^{*}$ since $\alpha(y)$ should converge to zero at a slower rate than $\|\hat{y}\|_{Y}^{2}$. We are not able to prove (3.6.1), but in the next chapter we present some preliminary results. 



\section{Chapter 4}

\section{Multiplicative regularization for}

\section{infinite-dimensional problems}

\subsection{Analysis of multiplicative regularization for infinite-dimensional problems}

Let $X$ and $Y$ be Hilbert spaces and let $T: X \rightarrow Y$ be a nonzero bounded linear operator. We wish to estimate $x \in X$ that satisfies $T x=y$, which is an inverse problem when $R(T)$ fails to be closed. Multiplicative regularization is based on minimizing the function

$$
J(x ; y)=\frac{1}{2}\|T x-y\|_{Y}^{2}\|x\|_{X}^{2} .
$$


Assuming $y$ is near $y^{*}$, we try to find a local minimizer of $J$ near $x^{*}=T^{\dagger} y^{*}$. When $y \notin R(T), x$ is a nontrivial stationary point of $J(\cdot ; y)$ if and only if $x=x_{\alpha, y}$, where

$$
\alpha=\frac{\left\|T x_{\alpha, y}-y\right\|_{Y}^{2}}{\left\|x_{\alpha, y}\right\|_{X}^{2}} .
$$

We want to study (4.1.1) or equivalently $\left\|T x_{\alpha, y}-y\right\|_{Y}^{2}-\alpha\left\|x_{\alpha, y}\right\|_{X}^{2}=0$.

Let $y \in Y$. Write $y=\bar{y}+\hat{y}$ where $\bar{y}=\operatorname{proj}_{\overline{R(T)}} y$ and $\hat{y}=\operatorname{proj}_{R(T)^{\perp}} y$. We define the function $F:[0, \infty) \times Y \rightarrow R$ by

$$
F(\alpha, y)= \begin{cases}\left\|T x_{\alpha, y}-\bar{y}\right\|_{Y}^{2}-\alpha\left\|x_{\alpha, y}\right\|_{X}^{2}+\|\hat{y}\|_{Y}^{2}, & \alpha>0, \\ \|\hat{y}\|^{2} & \alpha=0,\end{cases}
$$

We want to show that for all $y$ near $y^{*}$ and $y \notin \overline{R(T)}$, there exists $\alpha$ such that $F(\alpha, y)=0$. It turns out that we cannot use the implicit function theorem to prove this, because we cannot prove that $F$ is $C^{1}$ (though it is continuous).

We define the new function $f(\alpha, y)=\|\hat{y}\|_{Y}^{2}-F(\alpha, y)$, that is,

$$
f(\alpha, y)= \begin{cases}\alpha\left\|x_{\alpha, y}\right\|_{X}^{2}-\left\|T x_{\alpha, y}-\bar{y}\right\|_{Y}^{2}, & \alpha>0 \\ 0, & \alpha=0 .\end{cases}
$$

Then $F(\alpha, y)=0$ is equivalent to $f(\alpha, y)=\|\hat{y}\|_{Y}^{2}$. We need to show that the function 
$F$ is continuous. We start with the following important results.

Lemma 21. For each $y \in Y, T x_{\alpha, y} \rightarrow \bar{y}=\operatorname{proj}_{\overline{R(T)}}$ y as $\alpha \rightarrow 0^{+}$.

Proof. Since $T x_{\alpha, y} \in R(T)$, then by the Pythagorean theorem we have

$$
\left\|T x_{\alpha, y}-y\right\|_{Y}^{2}=\left\|T x_{\alpha, y}-\bar{y}+\bar{y}-y\right\|_{Y}^{2}=\left\|T x_{\alpha, y}-\bar{y}\right\|_{Y}^{2}+\|y-\bar{y}\|_{Y}^{2} .
$$

It follows that $\left\|T x_{\alpha, y}-y\right\|_{Y}^{2} \geq\|y-\bar{y}\|_{Y}^{2}$ and it can be shown that $\left\|T x_{\alpha, y}-y\right\|_{Y}^{2}$ is non-increasing as $\alpha \rightarrow 0^{+}$(see Proposition 5.24 in [12]). Thus

$$
\lim _{\alpha \rightarrow 0^{+}}\left\|T x_{\alpha, y}-y\right\|_{Y}^{2}=M \geq\|y-\bar{y}\|_{Y}^{2} .
$$

We need to show that $M=\|y-\bar{y}\|_{Y}^{2}$. By way of contradiction, suppose that $M>$ $\|y-\bar{y}\|_{Y}^{2}$. Define $\epsilon$ to be $\epsilon=M-\|y-\bar{y}\|_{Y}^{2}$. Choose $x \in X$ such that $\|T x-y\|_{Y}^{2}<M-\frac{\epsilon}{2}$. Then for all $\alpha>0$ sufficiently small we have

$\|T x-y\|_{Y}^{2}+\alpha\|x\|_{X}^{2}<M-\frac{\epsilon}{2}+\alpha\|x\|_{X}^{2}<M<\|y-\bar{y}\|_{Y}^{2} \leq\left\|T x_{\alpha, y}-y\right\|_{Y}^{2} \leq\left\|T x_{\alpha, y}-y\right\|_{Y}^{2}+\alpha\left\|x_{\alpha, y}\right\|_{X}^{2}$,

which is a contradiction. This shows that $M=\|y-\bar{y}\|_{Y}^{2}$ and

$$
\lim _{\alpha \rightarrow 0^{+}}\left\|T x_{\alpha, y}-y\right\|_{Y}^{2}=\|y-\bar{y}\|_{Y}^{2} .
$$

We have that $\left\|T x_{\alpha, y}-y\right\|_{Y}^{2}=\left\|T x_{\alpha, y}-\bar{y}\right\|_{Y}^{2}+\|y-\bar{y}\|_{Y}^{2}$ and as $\alpha \rightarrow 0^{+},\left\|T x_{\alpha, y}-y\right\|_{Y}^{2} \rightarrow$ $\|y-\bar{y}\|_{Y}^{2}$, thus it follows that $\left\|T x_{\alpha, y}-\bar{y}\right\|_{Y}^{2} \rightarrow 0$ as $\alpha \rightarrow 0^{+}$, i.e., $T x_{\alpha, y} \rightarrow \bar{y}$ as 
$\alpha \rightarrow 0^{+}$.

Lemma 22. For all $y \in Y, \sqrt{\alpha} N_{\alpha}^{-1} T^{*} y \rightarrow 0$ as $\alpha \rightarrow 0^{+}$.

Proof. We have that $\left\|T x_{\alpha, y}-y\right\|_{Y}^{2}+\left\|T x_{\alpha, y}\right\|_{Y}^{2}+2 \alpha\left\|x_{\alpha, y}\right\|_{X}^{2}=\|y\|_{Y}^{2}$. Also it follows from the previous lemma that for each $y \in Y$ as $\alpha \rightarrow 0^{+}, T x_{\alpha, y} \rightarrow \bar{y}$. Thus as $\alpha \rightarrow 0^{+}$,

$$
\begin{aligned}
2 \alpha\left\|x_{\alpha, y}\right\|_{X}^{2} & =\|y\|_{Y}^{2}-\left\|T x_{\alpha, y}-y\right\|_{Y}^{2}-\left\|T x_{\alpha, y}\right\|_{Y}^{2} \\
& \rightarrow\|y\|_{Y}^{2}-\|y-\bar{y}\|_{Y}^{2}-\|\bar{y}\|_{Y}^{2} \\
& =\|y\|_{Y}^{2}-\|\hat{y}\|_{Y}^{2}-\|\bar{y}\|_{Y}^{2}=0 .
\end{aligned}
$$

Therefore $\alpha\left\|x_{\alpha, y}\right\|_{X}^{2}=\left\|\sqrt{\alpha} N_{\alpha}^{-1} T^{*} y\right\|_{X}^{2} \rightarrow 0$ as $\alpha \rightarrow 0^{+}$and the proof is complete.

Now we can prove that the function $F$ is continuous.

Theorem 23. The function $F$ is continuous.

Proof. It is straightforward to prove that $F$ is continuous on $(0, \infty) \times Y$. We need to show that for all $y_{0} \in Y, \lim _{(\alpha, y) \rightarrow\left(0, y_{0}\right)} F(\alpha, y)=F\left(0, y_{0}\right)$. We have

$$
\lim _{(\alpha, y) \rightarrow\left(0, y_{0}\right)} F(\alpha, y)=\lim _{(\alpha, y) \rightarrow\left(0, y_{0}\right)}\left(\left\|T x_{\alpha, y}-y\right\|_{Y}^{2}-\alpha\left\|x_{\alpha, y}\right\|_{X}^{2}\right)
$$


We can see that $\lim _{(\alpha, y) \rightarrow\left(0, y_{0}\right)}\left\|T x_{\alpha, y}-y\right\|_{Y}^{2}=\left\|\hat{y}_{0}\right\|_{Y}^{2}$, since

$$
y-T x_{\alpha, y}=y-T N_{\alpha}^{-1} T^{*} y=y-T N_{\alpha}^{-1} T^{*}\left(y-y_{0}\right)-T N_{\alpha}^{-1} T^{*} y_{0}
$$

Now as $(\alpha, y) \rightarrow\left(0, y_{0}\right), T N_{\alpha}^{-1} T^{*}\left(y-y_{0}\right) \rightarrow 0$ since $\left\|T N_{\alpha}^{-1} T^{*}\right\| \leq 1$ and $T N_{\alpha}^{-1} T^{*} y_{0} \rightarrow$ $\bar{y}_{0}$ as $\alpha \rightarrow 0$. Thus we have

$$
y-T x_{\alpha, y} \rightarrow y_{0}-\bar{y}_{0}=\hat{y}_{0}, \text { as }(\alpha, y) \rightarrow\left(0, y_{0}\right)
$$

Also $\lim _{(\alpha, y) \rightarrow\left(0, y_{0}\right)} \alpha\left\|x_{\alpha, y}\right\|_{X}^{2}=0$. That is true because

$$
\sqrt{\alpha} x_{\alpha, y}=\sqrt{\alpha} N_{\alpha}^{-1} T^{*}\left(y-y_{0}\right)+\sqrt{\alpha} N_{\alpha}^{-1} T^{*} y_{0} \rightarrow 0 \text { as }(\alpha, y) \rightarrow\left(0, y_{0}\right) .
$$

Therefore,

$$
\lim _{(\alpha, y) \rightarrow\left(0, y_{0}\right)} F(\alpha, y)=\left\|\hat{y}_{0}\right\|_{Y}^{2}=F\left(0, y_{0}\right)
$$

and the proof is complete.

Since $F$ is continuous on $[0, \infty) \times Y$ and $f(\alpha, y)=\|\hat{y}\|_{Y}^{2}-F(\alpha, y)$, it follows that $f$ is continuous on $[0, \infty) \times Y$. For $y^{*} \in R(T)$ we have $f\left(\alpha, y^{*}\right)=\alpha\left\|x_{\alpha, y^{*}}\right\|_{X}^{2}-\| T x_{\alpha, y^{*}}-$ $y^{*} \|_{Y}^{2}$. Then clearly

$$
\lim _{\alpha \rightarrow 0^{+}} f\left(\alpha, y^{*}\right)=-\left\|T T^{\dagger} y^{*}-y^{*}\right\|_{Y}^{2}=0
$$


and also

$$
\lim _{\alpha \rightarrow \infty} f\left(\alpha, y^{*}\right)=-\left\|y^{*}\right\|_{Y}^{2} .
$$

We will show that $f\left(\alpha, y^{*}\right)>0$ for all $\alpha>0$ sufficiently small. We have

$$
\begin{aligned}
f\left(\alpha, y^{*}\right) & =\alpha\left\|x_{\alpha, y^{*}}\right\|_{X}^{2}-\left\|T x_{\alpha, y^{*}}-y^{*}\right\|_{Y}^{2}=\alpha\left(\left\|x_{\alpha, y^{*}}\right\|_{X}^{2}-\alpha^{-1}\left\|T x_{\alpha, y^{*}}-y^{*}\right\|_{Y}^{2}\right) \\
& =\alpha\left(\left\|x_{\alpha, y^{*}}\right\|_{X}^{2}-\alpha^{-1}\left\|T N_{\alpha}^{-1} T^{*} T x_{0, y^{*}}-T x_{0, y^{*}}\right\|_{Y}^{2}\right) \\
& =\alpha\left(\left\|x_{\alpha, y^{*}}\right\|_{X}^{2}-\alpha^{-1}\left\|T\left(I-\alpha N_{\alpha}^{-1}\right) x_{0, y^{*}}-T x_{0, y^{*}}\right\|_{Y}^{2}\right) \\
& =\alpha\left(\left\|x_{\alpha, y^{*}}\right\|_{X}^{2}-\alpha^{-1}\left\|\alpha T N_{\alpha}^{-1} x_{0, y^{*}}\right\|_{Y}^{2}\right) \\
& =\alpha\left(\left\|x_{\alpha, y^{*}}\right\|_{X}^{2}-\alpha\left\|T N_{\alpha}^{-1} x_{0, y^{*}}\right\|_{Y}^{2}\right) \\
& \geq \alpha\left(\left\|x_{\alpha, y^{*}}\right\|_{X}^{2}-\alpha\left\|T N_{\alpha}^{-1}\right\|^{2}\left\|x_{0, y^{*}}\right\|_{Y}^{2}\right) \\
& \geq \alpha\left(\left\|x_{\alpha, y^{*}}\right\|_{X}^{2}-\frac{1}{2}\left\|x_{0, y^{*}}\right\|_{X}^{2}\right) \quad\left(\left\|T N_{\alpha}^{-1}\right\|^{2} \leq \frac{1}{2 \alpha}\right) .
\end{aligned}
$$

Then since $\lim _{\alpha \rightarrow 0^{+}}\left(\left\|x_{\alpha, y^{*}}\right\|_{X}^{2}-\frac{1}{2}\left\|x_{0, y^{*}}\right\|_{X}^{2}\right)=\frac{1}{2}\left\|x_{0, y^{*}}\right\|_{X}^{2}>0$, it follows that $f\left(\alpha, y^{*}\right)>$ 0 for all $\alpha>0$ sufficiently small. Therefore there exists $\hat{\alpha}>0$ such that $f\left(\alpha, y^{*}\right)>0$ for all $\alpha \in(0, \hat{\alpha})$. We define $\alpha^{*}$ to be

$$
\alpha^{*}=\arg \max \left\{f\left(\alpha, y^{*}\right) \mid \alpha \in(0, \hat{\alpha})\right\} .
$$

Theorem 24. There exists $\epsilon>0$ such that for all $y \in B_{\epsilon_{1}}\left(y^{*}\right) \backslash \overline{R(T)}$, there exists $\alpha \in\left(0, \alpha^{*}\right)$ satisfying $f(\alpha, y)=\|\hat{y}\|_{Y}^{2}$. 
Proof. If $\epsilon_{1}$ is sufficiently small and $y \in B_{\epsilon_{1}}\left(y^{*}\right) \backslash \overline{R(T)}$, then $f(0, y)=0$ and $f\left(\alpha^{*}, y\right) \geq$ $\frac{1}{2} f\left(\alpha^{*}, y^{*}\right)$. We define $\epsilon_{2}=\sqrt{\frac{1}{2} f\left(\alpha^{*}, y^{*}\right)}$ and consider $\epsilon=\min \left\{\epsilon_{1}, \epsilon_{2}\right\}$. Now if $y \epsilon$ $B_{\epsilon}\left(y^{*}\right) \backslash \overline{R(T)}$, then we have $f(0, y)=0, f\left(\alpha^{*}, y\right) \geq \epsilon_{2}^{2}>0$, and $\|\hat{y}\|_{Y}^{2}<\epsilon_{2}^{2}$. By intermediate value theorem, since $f(0, y)<\|\hat{y}\|_{Y}^{2}<f\left(\alpha^{*}, y\right)$, there exists $0<\bar{\alpha}<\alpha^{*}$ such that $f(\bar{\alpha}, y)=\|\hat{y}\|_{Y}^{2}$.

By Theorem 24, we can define $\alpha: B_{\epsilon}\left(y^{*}\right) \rightarrow\left[0, \alpha^{*}\right]$ such that $\alpha=\alpha(y)$ is the smallest positive solution of $f(\alpha, y)=\|\hat{y}\|_{Y}^{2}$.

Now, we prove the following important result.

Lemma 25. As $y \rightarrow y^{*}, \alpha(y) \rightarrow 0$.

Proof. Let $\left\{y_{k}\right\} \subset Y, y_{k} \rightarrow y^{*}, \alpha_{k}=\alpha\left(y_{k}\right)$ for all $k \in \mathbb{Z}^{+}$. We have

$$
f\left(\alpha_{k}, y_{k}\right)=\|\hat{y}\|_{Y}^{2} \Leftrightarrow F\left(\alpha_{k}, y_{k}\right)=0 \Leftrightarrow\left\|T x_{\alpha_{k}, y_{k}}-y_{k}\right\|_{Y}^{2}=\alpha_{k}\left\|x_{\alpha_{k}, y_{k}}\right\|_{X}^{2} .
$$

If $\left\{\alpha_{k}\right\}$ does not converge to 0 , then without loss of generality we can assume that $\alpha_{k} \rightarrow \bar{\alpha} \in\left(0, \alpha^{*}\right]$ as $y_{k} \rightarrow y^{*}$. Then as $\left(\alpha_{k}, y_{k}\right) \rightarrow\left(\bar{\alpha}, y^{*}\right)$, we have $F\left(\alpha_{k}, y_{k}\right) \rightarrow$ $F\left(\bar{\alpha}, y^{*}\right)$ and that implies $F\left(\bar{\alpha}, y^{*}\right)=0$, which contradicts the fact that $F\left(\alpha, y^{*}\right)>0$ for all $\alpha \in(0, \hat{\alpha})$. So we have $\alpha_{k} \rightarrow 0$ as $y_{k} \rightarrow y^{*}$.

We proved that for each $y$ sufficiently close to $y^{*}, y \notin \overline{R(T)}$, there exists $\alpha>0$ 
satisfying $F(\alpha, y)=0$.

We would like to study the possibility of determining the rate at which $\alpha=\alpha(y) \rightarrow 0$ as $y \rightarrow y^{*}$.

\subsection{Rate of convergence}

The standard theory of Tikhonov regularization for an infinite-dimensional problem

shows that if $\alpha=\alpha(y) \rightarrow 0$ as $y \rightarrow y^{*}$ and $\frac{\left\|y-y^{*}\right\|_{Y}^{2}}{\alpha} \leq C$, then $x_{\alpha, y} \rightarrow x_{0, y^{*}}$ weakly.

To see that, we have

$$
\begin{aligned}
\left\|x_{\alpha, y}-x_{0, y^{*}}\right\|_{X} & =\left\|x_{\alpha, y}-x_{\alpha, y^{*}}+x_{\alpha, y^{*}}-x_{0, y^{*}}\right\|_{X} \\
& \leq\left\|x_{\alpha, y}-x_{\alpha, y^{*}}\right\|_{X}+\left\|x_{\alpha, y^{*}}-x_{0, y^{*}}\right\|_{X} .
\end{aligned}
$$

It is clear that $\left\|x_{\alpha, y^{*}}-x_{0, y^{*}}\right\|_{X}$ is bounded as $\alpha \rightarrow 0$ since

$$
\begin{aligned}
\left\|x_{\alpha, y^{*}}-x_{0, y^{*}}\right\|_{X} & =\left\|N_{\alpha}^{-1} T^{*} y^{*}-x_{0, y^{*}}\right\|_{X}=\left\|N_{\alpha}^{-1} T^{*} T x_{0, y^{*}}-x_{0, y^{*}}\right\|_{X} \\
& =\left\|\left(I-\alpha N_{\alpha}^{-1}\right) x_{0, y^{*}}-x_{0, y^{*}}\right\|_{X}=\left\|\alpha N_{\alpha}^{-1} x_{0, y^{*}}\right\|_{X} \\
& \leq\left\|\alpha N_{\alpha}^{-1}\right\|\left\|x_{0, y^{*}}\right\|_{X} \leq\left\|x_{0, y^{*}}\right\|_{X} \leq \hat{C} .
\end{aligned}
$$

In fact, since $x_{\alpha, y^{*}} \rightarrow T^{\dagger} y^{*}$ strongly as $\alpha \rightarrow 0$, it follows that $\left\|x_{\alpha, y^{*}}-x_{0, y^{*}}\right\|_{X} \rightarrow 0$ 
as $\alpha \rightarrow 0$. Also we have

$$
\left\|x_{\alpha, y}-x_{\alpha, y^{*}}\right\|_{X} \leq\left\|N_{\alpha}^{-1} T^{*}\left(y-y^{*}\right)\right\|_{X} \leq \frac{1}{\sqrt{2 \alpha}}\left\|y-y^{*}\right\|_{Y} \leq \frac{1}{\sqrt{2}} C^{\frac{1}{2}} .
$$

Thus $\left\{x_{\alpha, y}\right\}$ is bounded as $y \rightarrow y^{*}$ and it can be shown that $x_{\alpha, y} \rightarrow x_{0, y^{*}}$ weakly under this condition. To show that, suppose $x_{\alpha, y} \rightarrow x \in X$ weakly. We will see that $x=x_{0, y^{*}}$. We have that $T^{*} y \rightarrow T^{*} y^{*}$ strongly (since $y \rightarrow y^{*}$ strongly and the operator $T^{*}$ is bounded) and also $T^{*} T x_{\alpha, y} \rightarrow T^{*} T x$ weakly (since $x_{\alpha, y} \rightarrow x$ weakly and the operator $T^{*} T$ is bounded). Moreover, since $\left\{x_{\alpha, y}\right\}$ is bounded, $\alpha x_{\alpha, y} \rightarrow 0$ strongly as $\alpha \rightarrow 0$. We have $T^{*} T x_{\alpha, y}+\alpha x_{\alpha, y}=T^{*} y$.

It follows that $T^{*} T x=T^{*} y^{*}$ and that implies $x$ is a least-square solution of $T x=y^{*}$. We have seen that $x_{\alpha, y} \in N(T)^{\perp}$ and since $N(T)^{\perp}$ is closed, then $x \in N(T)^{\perp}$. It follows that $x$ is the minimum-norm least-squares solution of $T x=y^{*}$, that is, $x=x_{0, y^{*}}$.

For an infinite-dimensional inverse problem, a purely a posteriori parameter choice method like multiplicative regularization cannot be convergent. We conjecture that if $\|\hat{y}\|_{Y} \geq \theta\left\|y-y^{*}\right\|_{Y}$ holds, then $\frac{\|\hat{y}\|_{Y}^{2}}{\alpha(y)} \leq C$ holds and $x_{\alpha, y} \rightarrow x_{0, y^{*}}$ weakly. The condition $\|\hat{y}\|_{Y} \geq \theta\left\|y-y^{*}\right\|_{Y}$ is necessary to ensure that each $y_{k}$ contains a significant component in $R(T)^{\perp}$. 


\subsubsection{Special cases}

We would like to show that $\frac{\|\hat{y}\|^{2}}{\alpha}$ is bounded above as $y \rightarrow y^{*}$. Unfortunately we are not able to prove that in general, but we can show that it is true for two special cases. First suppose that $\left\{y_{k}\right\} \subset Y, y_{k}=y^{*}+\hat{y}_{k}, \alpha_{k}=\alpha\left(y_{k}\right)$ for all $k \in \mathbb{Z}^{+}$and $y_{k} \rightarrow y^{*}$, as $k \rightarrow \infty$. Then we have

$$
\begin{aligned}
\frac{\left\|\hat{y}_{k}\right\|_{Y}^{2}}{\alpha_{k}} & =\left\|x_{\alpha_{k}, y_{k}}\right\|_{X}^{2}-\frac{1}{\alpha_{k}}\left\|T x_{\alpha_{k}, y_{k}}-y_{k}\right\|_{Y}^{2} \leq\left\|x_{\alpha_{k}, y_{k}}\right\|_{X}^{2} \\
& =\sum_{i=1}^{\infty} \frac{\sigma_{i}^{2}}{\left(\sigma_{i}^{2}+\alpha_{k}\right)^{2}}\left|\left\langle y_{k}, \psi_{i}\right\rangle_{Y}\right|^{2}=\sum_{i=1}^{\infty} \frac{\sigma_{i}^{2}}{\left(\sigma_{i}^{2}+\alpha_{k}\right)^{2}}\left|\left\langle y^{*}+\hat{y}_{k}, \psi_{i}\right\rangle_{Y}\right|^{2} \\
& =\sum_{i=1}^{\infty} \frac{\sigma_{i}^{2}}{\left(\sigma_{i}^{2}+\alpha_{k}\right)^{2}}\left|\left\langle y^{*}, \psi_{i}\right\rangle_{Y}\right|^{2}+\sum_{i=1}^{\infty} \frac{\sigma_{i}^{2}}{\left(\sigma_{i}^{2}+\alpha_{k}\right)^{2}}\left|\left\langle\hat{y}_{k}, \psi_{i}\right\rangle_{Y}\right|^{2} \\
& =\left\|x_{\alpha_{k}, y^{*}}\right\|_{X}^{2} \leq\left\|x_{0, y^{*}}\right\|_{X}^{2} \text { since }\left\langle\hat{y}_{k}, \psi_{i}\right\rangle_{Y}=0 \forall i .
\end{aligned}
$$

Thus $\frac{\left\|\hat{y}_{k}\right\|_{Y}^{2}}{\alpha_{k}} \leq C$ as $y_{k} \rightarrow y^{*}$, where $C=\left\|x_{0, y^{*}}\right\|_{X}^{2}$.

For our second special case we suppose $\left\{y_{k}\right\} \subset Y, y_{k}=y^{*}+\sigma_{k} \psi_{k}+\hat{y}_{k}, \alpha_{k}=\alpha\left(y_{k}\right)$ for all $k \in \mathbb{Z}^{+}$and $\hat{y}_{k} \rightarrow 0$, as $k \rightarrow \infty$. Then we have

$$
\begin{aligned}
\frac{\left\|\hat{y}_{k}\right\|_{Y}^{2}}{\alpha_{k}} & =\left\|x_{\alpha_{k}, y_{k}}\right\|_{X}^{2}-\frac{1}{\alpha_{k}}\left\|T x_{\alpha_{k}, y_{k}}-y_{k}\right\|_{Y}^{2} \leq\left\|x_{\alpha_{k}, y_{k}}\right\|_{X}^{2}=\sum_{i=1}^{\infty} \frac{\sigma_{i}^{2}}{\left(\sigma_{i}^{2}+\alpha_{k}\right)^{2}}\left|\left\langle y_{k}, \psi_{i}\right\rangle_{Y}\right|^{2} \\
& =\sum_{i=1}^{\infty} \frac{\sigma_{i}^{2}}{\left(\sigma_{i}^{2}+\alpha_{k}\right)^{2}}\left|\left\langle y^{*}+\sigma_{k} \psi_{k}+\hat{y}_{k}, \psi_{i}\right\rangle_{Y}\right|^{2} \\
& =\sum_{i=1}^{\infty} \frac{\sigma_{i}^{2}}{\left(\sigma_{i}^{2}+\alpha_{k}\right)^{2}}\left|\left\langle y^{*}+\sigma_{k} \psi_{k}, \psi_{i}\right\rangle_{Y}\right|^{2} \text { since }\left\langle\hat{y}_{k}, \psi_{i}\right\rangle_{Y}=0 \forall i \\
& =\sum_{i=1}^{\infty} \frac{\sigma_{i}^{2}}{\left(\sigma_{i}^{2}+\alpha_{k}\right)^{2}}\left|\left\langle y^{*}, \psi_{i}\right\rangle_{Y}\right|^{2}+\frac{\sigma_{k}^{4}}{\left(\sigma_{k}^{2}+\alpha_{k}\right)^{2}}+\frac{2 \sigma_{k}^{3}}{\left(\sigma_{k}^{2}+\alpha_{k}\right)^{2}}\left\langle y^{*}, \psi_{k}\right\rangle_{Y} \\
& =\left\|x_{\alpha_{k}, y^{*}}\right\|_{X}^{2}+\frac{\sigma_{k}^{4}}{\left(\sigma_{k}^{2}+\alpha_{k}\right)^{2}}+\frac{2 \sigma_{k}^{3}}{\left(\sigma_{k}^{2}+\alpha_{k}\right)^{2}}\left|\left\langle y^{*}, \psi_{k}\right\rangle_{Y}\right| .
\end{aligned}
$$


The last step follows from the fact $\left\langle\hat{y}_{k}, \psi_{k}\right\rangle_{Y}=0$ since $\hat{y}_{k}$ is orthogonal to $\operatorname{col}(A)$. It can be seen that $\frac{\left\|\hat{y}_{k}\right\|_{Y}^{2}}{\alpha_{k}}$ is bounded above since $\left\|x_{\alpha_{k}, y^{*}}\right\|_{X}^{2}$ is bounded by $\left\|x_{0, y^{*}}\right\|_{X}^{2}$, $\frac{\sigma_{k}^{4}}{\left(\sigma_{k}^{2}+\alpha_{k}\right)^{2}}$ is bounded by 1 and also

$$
\frac{2 \sigma_{k}^{3}}{\left(\sigma_{k}^{2}+\alpha_{k}\right)^{2}}\left|\left\langle y^{*}, \psi_{k}\right\rangle_{Y}\right|=\frac{2 \sigma_{k}^{4}}{\left(\sigma_{k}^{2}+\alpha_{k}\right)^{2}} \frac{\left|\left\langle y^{*}, \psi_{k}\right\rangle_{Y}\right|}{\sigma_{k}} \rightarrow 0
$$

Therefore $\frac{\left\|\hat{y}_{k}\right\|_{Y}^{2}}{\alpha_{k}}$ is bounded above by a constant $C$ where $C>0$. Also we can show that by imposing the condition $\left\|\bar{y}_{k}-y_{k}^{*}\right\|<\theta\left\|\hat{y}_{k}\right\|$ (for some $\theta>0$ ), $\frac{\alpha}{\left\|\hat{y}_{k}\right\|_{Y}^{2}}$ is bounded above. To see that we have

$$
\begin{aligned}
\frac{\left\|\hat{y}_{k}\right\|_{Y}^{2}}{\alpha} & =\left\|x_{\alpha_{k}, y_{k}^{*}}\right\|_{X}^{2}-\frac{1}{\alpha}\left\|T x_{\alpha_{k}, y_{k}^{*}}-y_{k}^{*}\right\|_{Y}^{2} \\
& \geq\left\|x_{\alpha_{k}, y_{k}^{*}}\right\|_{X}^{2}-\frac{1}{\alpha}\left\|\bar{y}_{k}-y_{k}^{*}\right\|_{Y}^{2} \\
& \geq\left\|x_{\alpha_{k}, y_{k}^{*}}\right\|_{X}^{2}-\frac{\theta\left\|\hat{y}_{k}\right\|_{Y}^{2}}{\alpha} .
\end{aligned}
$$

It follows that $(1+\theta) \frac{\left\|\hat{y}_{k}\right\|_{Y}^{2}}{\alpha} \geq\left\|x_{\alpha_{k}, y_{k}^{*}}\right\|_{X}^{2}$ and thus

$$
\frac{\alpha}{\left\|\hat{y}_{k}\right\|_{Y}^{2}} \leq \frac{(1+\theta)}{\left\|x_{\alpha_{k}, y_{k}^{*}}\right\|_{X}^{2}} \leq \hat{C} \text { as } y_{k} \rightarrow y^{*}
$$

where $\hat{C}>0$. Thus $0<\hat{C} \leq \frac{\left\|\hat{y}_{k}\right\|_{Y}^{2}}{\alpha} \leq C$. 


\subsubsection{Counter-example}

Now with a counter-example, we can show that imposing the condition

$$
\|\hat{y}\|_{Y} \geq \theta\left\|y-y^{*}\right\|_{Y}
$$

is necessary. In the previous section, we proved that for all $y$ sufficiently close to $y^{*}$ and $y \notin \overline{R(T)}$, there exists $\alpha=\alpha(y)>0$ satisfying $f(\alpha, y)=\|\hat{y}\|_{Y}^{2}$, that is, there exists $\alpha=\alpha(y)>0$ satisfying $\alpha=\frac{\|\hat{y}\|_{Y}^{2}}{\left\|x_{\alpha, y}\right\|_{X}^{2}-\alpha^{-1}\left\|T x_{\alpha, y}-\bar{y}\right\|_{Y}^{2}}$.

We have

$$
\begin{aligned}
& \alpha-\frac{\|\hat{y}\|_{Y}^{2}}{\left\|x_{0, y^{*}}\right\|_{X}^{2}}=\frac{\|\hat{y}\|_{Y}^{2}}{\left\|x_{\alpha, y^{*}}\right\|_{X}^{2}-\alpha^{-1}\left\|T x_{\alpha, y^{*}}-y^{*}\right\|_{Y}^{2}}-\frac{\|\hat{y}\|_{Y}^{2}}{\left\|x_{0, y^{*}}\right\|_{X}^{2}} \\
& =\frac{\|\hat{y}\|_{Y}^{2}\left(\left\|x_{0, y^{*}}\right\|_{X}^{2}-\left(\left\|x_{\alpha, y^{*}}\right\|_{X}^{2}-\alpha^{-1}\left\|T x_{\alpha, y^{*}}-y\right\|_{Y}^{2}\right)\right)}{\left\|x_{0, y^{*}}\right\|_{X}^{2}\left(\left\|x_{\alpha, y^{*}}\right\|_{X}^{2}-\alpha^{-1}\left\|T x_{\alpha, y^{*}}-y^{*}\right\|_{Y}^{2}\right)} \\
& =\frac{\|\hat{y}\|_{Y}^{2}}{\left\|x_{0, y^{*}}\right\|_{X}^{2}}\left(\frac{\left\|x_{0, y^{*}}\right\|_{X}^{2}-\left(\left\|x_{\alpha, y^{*}}\right\|_{X}^{2}-\alpha^{-1}\left\|T x_{\alpha, y^{*}}-y^{*}\right\|_{Y}^{2}\right)}{\left\|x_{\alpha, y^{*}}\right\|_{X}^{2}-\alpha^{-1}\left\|T x_{\alpha, y^{*}}-y^{*}\right\|_{Y}^{2}}\right) \text {, }
\end{aligned}
$$

and thus it follows that

$$
\begin{aligned}
& \frac{\|\hat{y}\|_{Y}^{2}}{\left\|x_{\alpha, y^{*}}\right\|_{X}^{2}-\alpha^{-1}\left\|T x_{\alpha, y^{*}}-y^{*}\right\|_{Y}^{2}}=\frac{\|\hat{y}\|_{Y}^{2}}{\left\|x_{0, y^{*}}\right\|_{X}^{2}}+\frac{\|\hat{y}\|_{Y}^{2}}{\left\|x_{0, y^{*}}\right\|_{X}^{2}}\left(\frac{\left\|x_{0, y^{*}}\right\|_{X}^{2}-\left(\left\|x_{\alpha, y^{*}}\right\|_{X}^{2}-\alpha^{-1}\left\|T x_{\alpha, y^{*}}-y^{*}\right\|_{Y}^{2}\right)}{\left\|x_{\alpha, y^{*}}\right\|_{X}^{2}-\alpha^{-1}\left\|T x_{\alpha, y^{*}}-y^{*}\right\|_{Y}^{2}}\right) \\
& =\frac{\|\hat{y}\|_{Y}^{2}}{\left\|x_{0, y^{*}}\right\|_{X}^{2}}\left(1+\frac{\left\|x_{0, y^{*}}\right\|_{X}^{2}-\left\|x_{\alpha, y^{*}}\right\|_{X}^{2}-\alpha^{-1}\left\|T x_{\alpha, y^{*}}-y^{*}\right\|_{Y}^{2}}{\left\|x_{\alpha, y^{*}}\right\|_{X}^{2}-\alpha^{-1}\left\|T x_{\alpha, y^{*}}-y^{*}\right\|_{Y}^{2}}\right) .
\end{aligned}
$$


We can choose $\left\{y_{k}^{*}\right\} \subset R(T)$ such that as $k \rightarrow \infty, y_{k}^{*} \rightarrow y^{*}$ and $\left\|x_{0, y_{k}^{*}}\right\|^{2} \rightarrow \infty$. To see that, suppose $y_{k}^{*}=y^{*}+\sqrt{\sigma_{k}} \psi_{k}$. Then we have

$$
\begin{aligned}
x_{0, y_{k}^{*}} & =T^{\dagger} y_{k}^{*}=T^{\dagger}\left(y^{*}+\sqrt{\sigma_{k}} \psi_{k}\right)=T^{\dagger} y^{*}+T^{\dagger} \sqrt{\sigma_{k}} \psi_{k} \\
& =x_{0, y^{*}}+\frac{\sqrt{\sigma_{k}}}{\sigma_{k}} \psi_{k}=x_{0, y^{*}}+\frac{1}{\sqrt{\sigma_{k}}} \psi_{k} .
\end{aligned}
$$

Clearly as $k \rightarrow \infty, \sigma_{k} \rightarrow 0, y_{k}^{*} \rightarrow y^{*}$ and $\left\|x_{0, y_{k}^{*}}\right\|^{2} \rightarrow \infty$. Now for each $k$, choose $y_{k}$ as

$$
y_{k}=y_{k}^{*}+\hat{y}_{k}=y^{*}+\sqrt{\sigma_{k}} \psi_{k}+\hat{y}_{k}
$$

where $\hat{y}_{k}$ is sufficiently small such that

$$
\alpha\left(y_{k}\right)=\frac{\left\|\hat{y}_{k}\right\|_{Y}^{2}}{\left\|x_{0, y_{k}^{*}}\right\|_{X}^{2}}\left(1+\theta_{k}\right),\left|\theta_{k}\right|<\frac{1}{k} .
$$

To see that, we have

$$
\begin{aligned}
\theta_{k} & =\frac{\left\|x_{0, y_{k}^{*}}\right\|_{X}^{2}-\left\|x_{\alpha_{k}, y_{k}^{*}}\right\|_{X}^{2}-\alpha_{k}^{-1}\left\|T x_{\alpha_{k}, y_{k}^{*}}-y_{k}^{*}\right\|_{Y}^{2}}{\left\|x_{\alpha_{k}, y_{k}^{*}}\right\|_{X}^{2}-\alpha_{k}^{-1}\left\|T x_{\alpha_{k}, y_{k}^{*}}-y_{k}^{*}\right\|_{Y}^{2}} \\
& =\frac{\sum_{n=1}^{\infty} \frac{3 \alpha_{k} \sigma_{n}^{2}+\alpha_{k}^{2}}{\left(\sigma_{n}^{2}+\alpha_{k}\right)^{2}} \frac{\left|\left\langle y_{k}^{*}, \psi_{n}\right\rangle\right|^{2}}{\sigma_{n}^{2}}}{\sum_{n=1}^{\infty} \frac{\sigma_{n}^{2}-\alpha_{k}^{2}}{\left(\sigma_{n}^{2}+\alpha_{k}\right)^{2}}\left|\left\langle y_{k}^{*}, \psi_{n}\right\rangle\right|^{2}} \\
& \leq \frac{\sum_{n=1}^{\infty} \frac{3 \sigma_{n}^{2}+\alpha_{k}}{\left(\sigma_{n}^{2}+\alpha_{k}\right)} \frac{\left|\left\langle y_{k}^{*}, \psi_{n}\right\rangle\right|^{2}}{\sigma_{n}^{2}}}{\sum_{n=1}^{\infty} \frac{\sigma_{n}^{2}-\alpha_{k}^{2}}{\left(\sigma_{n}^{2}+\alpha_{k}\right)^{2}}\left|\left\langle y_{k}^{*}, \psi_{n}\right\rangle\right|^{2}} .
\end{aligned}
$$

It follows that for each fixed $k, \theta_{k} \rightarrow 0$ as $\hat{y}_{k} \rightarrow 0$ (because $\alpha_{k} \rightarrow 0$ as $\hat{y}_{k} \rightarrow 0$ ). 
We also have that

$$
\left\|y_{k}-y_{k}^{*}\right\|_{Y}^{2}=\left\|y_{k}-\bar{y}_{k}\right\|_{Y}^{2}+\left\|\bar{y}_{k}-y_{k}^{*}\right\|_{Y}^{2}=\left\|y_{k}-\bar{y}_{k}\right\|_{Y}^{2}+\left\|\hat{y}_{k}\right\|_{Y}^{2}
$$

and clearly $\left\|\hat{y}_{k}\right\|_{Y}^{2} \leq\left\|y_{k}-y_{k}^{*}\right\|_{Y}^{2}$. We then have

$$
\frac{\alpha\left(y_{k}\right)}{\left\|y_{k}-y_{k}^{*}\right\|_{Y}^{2}} \leq \frac{\alpha\left(y_{k}\right)}{\left\|\hat{y}_{k}\right\|_{Y}^{2}}=\frac{1+\theta_{k}}{\left\|x_{0, y_{k}^{*}}\right\|_{X}^{2}} \rightarrow 0, \text { as } k \rightarrow \infty
$$

since $\left\|x_{0, y_{k}^{*}}\right\|^{2} \rightarrow \infty$ and $\theta_{k} \rightarrow 0$ as $k \rightarrow \infty$. Hence for this choice of $\left\{y_{k}\right\}, \frac{\left\|y_{k}-y_{k}^{*}\right\|_{Y}^{2}}{\alpha\left(y_{k}\right)}$ is not bounded. In general, we can see that $\frac{\|\hat{y}\|_{Y}^{2}}{\alpha(y)}$ need not be bounded if we do not impose the condition $\|\hat{y}\|_{Y} \geq \theta\left\|y-y^{*}\right\|_{Y}$.

\subsubsection{Numerical experiments}

In Chapter 3, we have shown that for a finite-dimensional problem,

$$
\lim _{b \rightarrow b^{*}} \frac{\|\hat{b}\|^{2}}{\alpha}=\lim _{b \rightarrow b^{*}}\left(\left\|x_{\alpha, b}\right\|^{2}-\alpha^{-1}\left\|A x_{\alpha, b}-\bar{b}\right\|^{2}\right)=\left\|x_{0, b^{*}}\right\|^{2}
$$

which implies as $b \rightarrow b^{*}, \frac{\|\hat{b}\|^{2}}{\alpha} \rightarrow\left\|x_{0, b^{*}}\right\|^{2}$. In this chapter, we will do some numerical experiments to test whether (4.2.1) seems to be true for infinite-dimensional problems.

We construct a noisy data function $y$ as $y=y^{*}+\delta z$, where $y^{*}$ is the exact data, $\delta$ is 
the noise level and $\mathrm{z}$ is a discontinuous function. In our example, the function $y$ does not belong to the $D\left(T^{\dagger}\right)$. Now by increasing $n$ (the dimension of the problem) and choosing $n$ sufficiently large, we get to compute the various quantities to 4 correct digits.

We consider some test problems from Hansen's Regularization Tools package [22]. We start with the test problem "baart", which is obtained by discretization of the first-kind Fredholm integral equation

$$
\int_{0}^{\pi} k(s, t) f(t) d t=g(s), \quad 0 \leq s \leq \frac{\pi}{2}
$$

where $k(s, t)=\exp (s \cos (t))$ and $f(t)=\sin (t)$. The right hand side is $g(s)=$ $2\left(\frac{\sinh (s)}{s}\right)+\delta z$. The function $z(s)$ is given by

$$
z= \begin{cases}s, & 0 \leq s \leq \frac{\pi}{4} \\ 2-2 s, & \frac{\pi}{4}<s \leq \frac{\pi}{2}\end{cases}
$$

The equation is discretized by the Galerkin method with orthonormal box functions. The dimension $n$ of the problem should be large enough in order to get accurate results, and we choose $n$ to be 1000 . The results for different noise levels are given in Table 4.1. The exact solution of the "baart" test problem satisfies $\left\|x^{*}\right\|^{2}=1.5708$. As we can see, as $y \rightarrow y^{*}, \frac{\|\hat{y}\|^{2}}{\alpha}$ appears to converge to $\left\|x_{0, y^{*}}\right\|^{2}$. 
Table 4.1

Numerical results for the infinite-dimensional test problem "baart"

\begin{tabular}{|c|c|c|c|c|}
\hline$\delta$ & $\alpha$ & $\|\hat{y}\|$ & $\frac{\|\hat{y}\|^{2}}{\alpha}$ & $\left\|x_{\alpha, y}\right\|^{2}$ \\
\hline $10^{-2}$ & $2.3556 \cdot 10^{-6}$ & $9.1723 \cdot 10^{-4}$ & 0.3571 & 1.8309 \\
\hline $10^{-3}$ & $1.4422 \cdot 10^{-8}$ & $9.1723 \cdot 10^{-5}$ & 0.5833 & 1.7123 \\
\hline $10^{-4}$ & $1.2021 \cdot 10^{-10}$ & $9.1723 \cdot 10^{-6}$ & 0.6999 & 1.6731 \\
\hline $10^{-5}$ & $1.1141 \cdot 10^{-12}$ & $9.1723 \cdot 10^{-7}$ & 0.7552 & 1.5943 \\
\hline $10^{-6}$ & $8.8572 \cdot 10^{-15}$ & $9.1723 \cdot 10^{-8}$ & 0.9499 & 1.6214 \\
\hline $10^{-7}$ & $7.2416 \cdot 10^{-17}$ & $9.1727 \cdot 10^{-9}$ & 1.1619 & 1.6763 \\
\hline $10^{-8}$ & $7.0916 \cdot 10^{-19}$ & $9.1723 \cdot 10^{-10}$ & 1.1864 & 1.5841 \\
\hline $10^{-9}$ & $6.4209 \cdot 10^{-21}$ & $9.1725 \cdot 10^{-11}$ & 1.3104 & 1.5854 \\
\hline $10^{-10}$ & $5.7308 \cdot 10^{-23}$ & $9.1750 \cdot 10^{-12}$ & 1.4689 & 1.6303 \\
\hline $10^{-11}$ & $5.4646 \cdot 10^{-25}$ & $9.3045 \cdot 10^{-13}$ & 1.5788 & 1.5824 \\
\hline
\end{tabular}

Our second example is the test problem "wing" which is obtained from discretization of the first-kind integral equation

$$
\int_{0}^{1} k(s, t) f(t) d t=g(s), \quad 0<s<1
$$

where $k(s, t)=t \exp \left(-s t^{2}\right)$, and the solution $f(t)$ is

$$
f(t)=\left\{\begin{array}{cc}
1, & \frac{1}{3}<t<\frac{2}{3}, \\
0, & \text { otherwise. }
\end{array}\right.
$$

The right hand side is given by $g(s)=\frac{\exp \left(-\frac{1}{9} s\right)-\exp \left(-\frac{4}{9} s\right)}{2 s}+\delta z$ where $z(s)$ is

$$
z= \begin{cases}s, & 0 \leq s \leq \frac{1}{2} \\ 2-2 s, & \frac{1}{2}<s \leq 1\end{cases}
$$


Table 4.2

Numerical results for the infinite-dimensional test problem "wing"

\begin{tabular}{|c|c|c|c|c|}
\hline$\delta$ & $\alpha$ & $\|\hat{y}\|$ & $\frac{\|\hat{y}\|^{2}}{\alpha}$ & $\left\|x_{\alpha, y}\right\|^{2}$ \\
\hline $10^{-2}$ & $1.04525 \cdot 10^{-4}$ & $1.5311 \cdot 10^{-3}$ & 0.0224 & 0.2720 \\
\hline $10^{-3}$ & $4.3871 \cdot 10^{-7}$ & $1.5311 \cdot 10^{-4}$ & 0.0534 & 0.2901 \\
\hline $10^{-4}$ & $5.6356 \cdot 10^{-9}$ & $1.5311 \cdot 10^{-5}$ & 0.0416 & 0.22758 \\
\hline $10^{-5}$ & $1.6054 \cdot 10^{-11}$ & $1.5311 \cdot 10^{-6}$ & 0.1460 & 0.32125 \\
\hline $10^{-6}$ & $1.7762 \cdot 10^{-13}$ & $1.5311 \cdot 10^{-7}$ & 0.1320 & 0.27201 \\
\hline $10^{-7}$ & $1.6345 \cdot 10^{-15}$ & $\cdot 1.531110^{-8}$ & 0.1434 & 0.2933 \\
\hline $10^{-8}$ & $1.0048 \cdot 10^{-17}$ & $1.5311 \cdot 10^{-9}$ & 0.2333 & 0.3327 \\
\hline $10^{-9}$ & $1.0623 \cdot 10^{-21}$ & $1.5311 \cdot 10^{-10}$ & 0.2206 & 0.2982 \\
\hline $10^{-10}$ & $1.0555 \cdot 10^{-21}$ & $1.5311 \cdot 10^{-11}$ & 0.2221 & 0.2976 \\
\hline $10^{-11}$ & $1.0281 \cdot 10^{-23}$ & $1.5311 \cdot 10^{-12}$ & 0.2280 & 0.3018 \\
\hline $10^{-12}$ & $7.5070 \cdot 10^{-26}$ & $1.5311 \cdot 10^{-13}$ & 0.3123 & 0.3196 \\
\hline
\end{tabular}

The equation is discretized by the Galerkin method with orthonormal box functions. The dimension of the problem $(n)$ is chosen to be 1000 . The numerical results for different noise levels are given in Table 4.3. The exact solution of the "wing" test problem is $\left\|x^{*}\right\|^{2}=0.3340$. One more time as $y \rightarrow y^{*}, \frac{\|\hat{y}\|^{2}}{\alpha}$ appears to converge to $\left\|x_{0, y^{*}}\right\|^{2}$

Our last example is "deriv2" which is obtained from discretization of the first-kind integral equation

$$
\int_{0}^{1} k(s, t) f(t) d t=g(s), \quad 0 \leq s \leq 1
$$

where

$$
k(s, t)= \begin{cases}s(t-1), & s<t \\ t(s-1), & \text { otherwise }\end{cases}
$$

and the exact solution is $f(t)=t$. The right hand side is $g(s)=\frac{1}{6}\left(s^{3}-s\right)+\delta z$. The 
Table 4.3

Numerical results for the infinite-dimensional test problem "deriv2"

\begin{tabular}{|c|c|c|c|c|}
\hline$\delta$ & $\alpha$ & $\|\hat{y}\|$ & $\frac{\|\hat{y}\|^{2}}{\alpha}$ & $\left\|x_{\alpha, y}\right\|^{2}$ \\
\hline $10^{-2}$ & $4.5601 \cdot 10^{-6}$ & $7.7204 \cdot 10^{-5}$ & 0.0013 & 0.3380 \\
\hline $10^{-3}$ & $5.8696 \cdot 10^{-9}$ & $7.7204 \cdot 10^{-6}$ & 0.0102 & 0.4763 \\
\hline $10^{-4}$ & $1.1966 \cdot 10^{-11}$ & $7.7204 \cdot 10^{-7}$ & 0.0498 & 0.4957 \\
\hline $10^{-5}$ & $1.5634 \cdot 10^{-14}$ & $7.7204 \cdot 10^{-8}$ & 0.3813 & 0.6055 \\
\hline $10^{-6}$ & $1.7359 \cdot 10^{-16}$ & $7.7206 \cdot 10^{-9}$ & 0.3434 & 0.3435 \\
\hline $10^{-7}$ & $1.7885 \cdot 10^{-18}$ & $7.7224 \cdot 10^{-10}$ & 0.3334 & 0.3334 \\
\hline $10^{-8}$ & $1.8048 \cdot 10^{-20}$ & $7.7563 \cdot 10^{-11}$ & 0.3333 & 0.3333 \\
\hline $10^{-9}$ & $2.6874 \cdot 10^{-22}$ & $9.4647 \cdot 10^{-12}$ & 0.3333 & 0.3333 \\
\hline $10^{-10}$ & $8.4016 \cdot 10^{-23}$ & $5.2920 \cdot 10^{-12}$ & 0.3333 & 0.3333 \\
\hline $10^{-11}$ & $8.1475 \cdot 10^{-23}$ & $5.2114 \cdot 10^{-12}$ & 0.3333 & 0.3333 \\
\hline $10^{-12}$ & $1.2790 \cdot 10^{-24}$ & $6.5295 \cdot 10^{-13}$ & 0.3333 & 0.3333 \\
\hline
\end{tabular}

function $z(s)$ is given by

$$
z= \begin{cases}s, & 0 \leq s \leq \frac{1}{2} \\ 2-2 s, & \frac{1}{2}<s \leq 1\end{cases}
$$

We discretize the equation by the Galerkin method with orthonormal box functions to get $T_{m n} x_{n}=y_{m}$ where $T_{m n} \in \mathbb{R}^{m \times n}, y_{m} \in \mathbb{R}^{m}$ and $x_{n} \in \mathbb{R}^{n}$ is to be estimated. For this problem $n$ is chosen to be 1000 and $m=2 n$. The numerical results are given in Table 4.2. The exact solution of the "deriv2" test problem is $\left\|x^{*}\right\|^{2}=0.3333$. Clearly as $y \rightarrow y^{*}, \frac{\|\hat{y}\|^{2}}{\alpha} \rightarrow\left\|x_{0, y^{*}}\right\|^{2}$. Notice that we do not expect multiplicative regularization to work for "deriv2" (in finite dimensions) because $\overline{R(T)}=Y$. However, this example suggests that, even for such a problem, multiplicative regularization might work if the problem is discretized with $m>n$. 


\subsection{Conclusions}

Based on our numerical results, we can see that for each test problem, by constructing a noisy data function $y$ (where $y \notin D\left(T^{\dagger}\right)$, discretizing the problem $T x=y$, choosing $n$ sufficiently large, we get to choose the accurate $\alpha$ and it appears that that as $y \rightarrow y^{*}, \frac{\|\hat{y}\|^{2}}{\alpha}$ converges to $\left\|x_{0, y^{*}}\right\|^{2}$

Our special cases and numerical examples suggest that

$$
y \rightarrow y^{*},\|\hat{y}\|_{Y} \geq \theta\left\|y-y^{*}\right\|_{Y} \Rightarrow \alpha(y) \rightarrow 0 \text { like }\|\hat{y}\|_{Y}^{2}
$$

However we are not able to prove the important result (4.3.1) in general. 



\section{Chapter 5}

\section{Conclusions}

\subsection{Summary and conclusions}

Inverse problems arise in many branches of science and engineering. In order to get a good approximation of the solution of this kind of problems, the use of regularization methods is required. Tikhonov regularization is one of the best methods for estimating the solutions of inverse problems. For a linear inverse problem $T x=y$, the objective function for Tikhonov regularization method is given by:

$$
\|T x-y\|_{Y}^{2}+\alpha\|x\|_{X}^{2} .
$$


The quality of the approximate solution computed by Tikhonov regularization depends on choosing a good regularization parameter $\alpha$.

The L-curve criterion is a purely a posteriori parameter choice method for the Tikhonov regularization method. This method works well most of the time, although there exist some problems in which the L-curve criterion does not perform properly. Because of difficulties associated with this method, for instance, we can not prove that the L-curve always has a corner; indeed, we have seen examples in which it does not. Therefore, we have investigated another parameter choice method.

Multiplicative regularization is a strategy for solving inverse problems. This method does not require any parameter selection strategies and, at the same time, can be considered as a parameter choice method for the Tikhonov regularization method. Multiplicative regularization is not an expensive method and is guaranteed to define a positive regularization parameter $\alpha$ provided provided $A \in \mathbb{R}^{m \times n}, \operatorname{col}(A)$ is a proper subspace of $\mathbb{R}^{m}$ and $b$ is sufficiently close to $b^{*}$. Under these conditions, multiplicative regularization performs very similarly to the L-curve method. Moreover, $\alpha \rightarrow 0$ at the same rate as $\|\hat{b}\|^{2}$ as $b \rightarrow b^{*}$.

We have presented some preliminary theoretical results for infinite-dimensional problems. For example, for all $y \in Y$ sufficiently close to $y^{*}$, there exists a positive regularization parameter $\alpha=\alpha(y)$ and also as $y \rightarrow y^{*}, \alpha(y) \rightarrow 0$. 


\subsection{Future work: plans and goals}

In general, for an infinite-dimensional inverse problem, a purely a posteriori parameter

choice method can not be convergent. However, if we conjecture that the results for finite-dimensional problems extend to infinite dimensions, namely, that

$$
y \rightarrow y^{*},\|\hat{y}\|_{Y} \geq \theta\left\|y-y^{*}\right\|_{Y} \Rightarrow \alpha(y) \rightarrow 0 \text { like }\|\hat{y}\|_{Y}^{2} .
$$

If our conjecture is true, then we obtain the weak convergence of $x_{\alpha, y}$ to $x^{*}$ under assumption (5.2.1).

We have not been able to prove (5.2.1), but we hope to prove this result in the future.

We also would like to study multiplicative regularization for nonlinear inverse problems. 



\section{Bibliography}

[1] T.M. Habashy A. Abubakr, P. M. van den Berg and H. Braunisch. A multiplicative regularization approach for deblurring problems. IEEE Transactions on Image processing, 13:1524-1532, 2001.

[2] A. Abubakr and P. M. van den Berg. Total variation as a multiplicative constraint for solving inverse problems. IEEE Transactions on Image processing, 10:1384$1392,2001$.

[3] A. B. Bakushinskii. Remarks on choosing a regularization parameter using the quasi-optimality and ratio criterion. USSR Comput. Math. and Math. Phys., 24:181-182, 1984.

[4] Fermin S Viloche Bazan. Fixed point iterations in determining the Tikhonov regularization parameter. Inverse Problems, 24:1-15, 2008.

[5] Fermin S Viloche Bazan. An improved fixed point algorithm for determining a Tikhonov regularization parameter. Inverse Problems, 25, 2009. 
[6] D. Calvetti, G. H. Golub, and L. Reichel. Estimation of the L-curve via Lanczos bidiagonalization. BIT, 39:603-619, 1999.

[7] D. Calvetti, L. Reichel, and A. Shuibi. L-curve and curvature bounds for Tikhonov regularization. Numerical Algorithms, 35:301-314, 2004.

[8] P J Mc Carthy. Direct analytic model of the L-curve for Tikhonov regularization parameter selection. Inverse Problems, 19:643-663, 2003.

[9] J. Longia Castellanos, Susana Gomez, and Valia Guerra. The triangle method for finding the corner of the L-curve. Applied Numerical Mathematics, 43:359-373, 2002.

[10] H. W. Engl and H. Gfrerer. A posteriori parameter choice for general regularization methods for solving linear ill-posed problems. Appl. Numer. Math., 4:395-417, 1988.

[11] H. W. Engl and Wilhelm Grever. Using the L-curve for determining optimal regularization parameters. Numer. Math., 69:25-31, 1994.

[12] H. W. Engl, M. Hanke, and A. Neubauer. Regularization of Inverse problems. Mathematics and its Applications, 1996.

[13] M. S. Gockenbach. Linear Inverse Problems and Tikhonov Regularization. To appear, 2015. 
[14] Gene H. Golub and Urs von Matt. Generalized Cross-Validation for Large Scale Problems. Comput. Graph. Stat, 6:1-34, 1995.

[15] C. W. Groetsch. The Theory of Tikhonov Regularization for Fredholm Fquations of the First Kind. Pitman, London, 1984.

[16] M. Hanke. Limitations of the L-curve method in ill-posed problems. BIT, 36:287301, 1996.

[17] M. Hanke and P.C. Hansen. Regularization methods for large-scale problems. Surveys on Mathematics for Industry, 3:253-315, 1993.

[18] P. C. Hansen. The truncated SVD as a method for regularization. BIT, 27:534$553,1987$.

[19] P. C. Hansen. Regularization, GSVD and truncated GSVD. BIT, 29:491-504, 1989.

[20] P. C. Hansen. The discrete Picard condition for discrete ill-posed problems. BIT, 30:658-672, 1990.

[21] P. C. Hansen. Analysis of discrete ill-posed problems by means of the L-curve. SIAM Review, 34:561-580, 1992.

[22] P. C. Hansen. Regularization tools: A Matlab package for analysis and solution of discrete ill-posed problems. Numerical Algorithms, 6:1-35, 1994. 
[23] P. C. Hansen. The L-curve and its use in the numerical treatment of inverse problems. Computational Inverse Problems in Electrocardiology, pages 119-142, 2001.

[24] P. C. Hansen and D. P. O'Leary. The use of the L-curve in the regularization of discrete ill-posed problems. SIAM J. Sci. Comput., 14:1487-1503, 1993.

[25] Per Christian Hansen. Rank-Deficient and Discrete ill-Posed Problems. SIAM, Philadelphia, 1998.

[26] Per Christian Hansen, Toke Koldborg Jensen, and Giuseppe Rodriguez. An adaptive pruning algorithm for the discrete L-curve criterion. Applied Computational Inverse Problems, 198:483-492, 2007.

[27] P. R. Johnston and R. M. Gulrajani. Selecting the corner in the L-curve approach to Tikhonov regularization. IEEE Transactions on biomedical engineering, 47:1293-1296, 2000.

[28] Misha E. Kilmer and Dianne P. O'Leary. Choosing regularization parameters in iterative methods for ill-posed problems. Siam J. Matrix Anal. Appl., 22:1204$1221,2001$.

[29] Dorota Krawczyk-Stando and Marek Rudnicki. Regularization parameter selection in discrete ill-posed problems-the use of the u-curve. Int. J. Appl. Math. Comput. Sci., 17:157-164, 2007. 
[30] K. H. Leem, G. Pelekanos, and F. S. V. Bazan. Fixed point iterations in determining a Tikhonov regularization parameter in Kirsch's factorization method. Applied Mathematics and Computations, 216:3747-3753, 2010.

[31] K. Miller. Least squares methods for ill-posed problems with a prescribed bound. SIAM J. Math. Anal, 1:52-74, 1970.

[32] V. A. Morozov. On the solution of functional equations by the method of regularization. Soviet Math. Dokl., 7:414-417, 1966.

[33] A. S. Nemirovskii. The regularization properties of the adjoint gradient method in ill-posed problems. USSR Comput. Math. Phys., 26:7-16, 1986.

[34] D. L. Phillips. A technique for the numerical solution of certain integral equations of the first kind. Association for Computing Machinery, 9:84-97, 1962.

[35] Teresa Reginska. Remarks on choosing a regularization parameter in Tikhonov method. preprint 499 IMPAN, Warszawa, 1992.

[36] Teresa Reginska. A regularization parameter in discrete ill-posed problems. SIAM J. Sci. Comput., 17:740-749, 1996.

[37] M. Rezghi and S. M. Hosseini. A new variant of L-curve for Tikhonov regularization. Journal of Computational and Applied Mathematics, 231:914-924, 2009. 
[38] José Alberto Orozco Rodríguez. Regularization methods for inverse problems. PhD thesis, University of Minnesota, Minneapolis, Minnesota, U.S.A., 2011.

[39] A. N. Tikhonov. Regularization of incorrectly posed problems. Soviet Mathematics, 4:1624-1627, 1963.

[40] T. Heath V. B. Golub and G. Wahba. Generalized cross-validation as a method for choosing a good ridge parameter. Technometrics, 21:215-223, 1979.

[41] P. M. van den Berg, A. L. van Broekhoven, and A. Abubakr. Extended contrast source inversion. Inverse problems, 15:1325-1344, 1999.

[42] C. R. Vogel. Non-convergence of the L-curve regularization parameter selection method. Inverse Problems, 12:535-547, 1996.

[43] G. Wahba. Practical approximate solutions to linear operator equations when the data are noisy. SIAM Journal on Numerical Analysis, 14:651-667, 1977. 